\title{
Moonshine, superconformal symmetry, and quantum error correction
}

\author{
Jeffrey A. Harvey ${ }^{a}$ and Gregory W. Moore ${ }^{b}$ \\ ${ }^{a}$ Enrico Fermi Institute and Department of Physics, University of Chicago, \\ 5620 Ellis Ave., Chicago IL 60637 \\ ${ }^{b}$ NHETC and Department of Physics and Astronomy, Rutgers University, \\ 126 Frelinghuysen Rd., Piscataway NJ 08855, U.S.A. \\ E-mail: j-harvey@uchicago.edu, gmoore@physics.rutgers.edu
}

ABSTRACT: Special conformal field theories can have symmetry groups which are interesting sporadic finite simple groups. Famous examples include the Monster symmetry group of a $c=24$ two-dimensional conformal field theory (CFT) constructed by Frenkel, Lepowsky and Meurman, and the Conway symmetry group of a $c=12$ CFT explored in detail by Duncan and Mack-Crane. The Mathieu moonshine connection between the K3 elliptic genus and the Mathieu group $M_{24}$ has led to the study of K3 sigma models with large symmetry groups. A particular K3 CFT with a maximal symmetry group preserving $(4,4)$ superconformal symmetry was studied in beautiful work by Gaberdiel, Taormina, Volpato, and Wendland [41]. The present paper shows that in both the GTVW and $c=12$ theories the construction of superconformal generators can be understood via the theory of quantum error correcting codes. The automorphism groups of these codes lift to symmetry groups in the CFT preserving the superconformal generators. In the case of the $N=1$ supercurrent of the GTVW model our result, combined with a result of T. Johnson-Freyd implies the symmetry group is the maximal subgroup of $M_{24}$ known as the sextet group. (The sextet group is also known as the holomorph of the hexacode.) Building on [41] the Ramond-Ramond sector of the GTVW model is related to the Miracle Octad Generator which in turn leads to a role for the Golay code as a group of symmetries of RR states. Moreover, $(4,1)$ superconformal symmetry suffices to define and decompose the elliptic genus of a K3 sigma model into characters of the $N=4$ superconformal algebra. The symmetry group preserving $(4,1)$ is larger than that preserving $(4,4)$.

KEywords: Conformal Field Models in String Theory, Conformal Field Theory, Discrete Symmetries

ARXIV EPRINT: 2003.13700 


\section{Contents}

1 Introduction 1

2 Codes and error correction $\quad 3$

2.1 Codes related to the Hamming code 4

2.2 The hexacode 5

2.3 The hexacode and the Golay code 9

$\begin{array}{ll}2.4 \text { Quantum codes } & 11\end{array}$

3 Supercurrents, states, and codes $\quad 13$

$\begin{array}{lll}3.1 & \text { The GTVW model } & 13\end{array}$

$3.2(4,4)$ superconformal algebra in the GTVW model 14

3.3 The hexacode representation of the $N=1$ supercurrent $\quad 16$

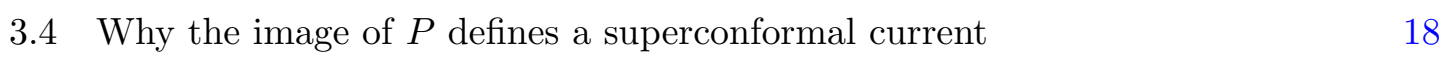

$\begin{array}{ll}3.5 & \text { The relation of the } N=4 \text { supercurrents to codes }\end{array}$

4 Relation to Mathieu moonshine $\quad 21$

4.1 Statement of Mathieu moonshine 21

4.2 The Mukai theorem 22

4.3 Quantum Mukai theorem 22

4.4 Evading a no-go theorem 23

5 Symmetries of supercurrents $\quad \mathbf{2 4}$

5.1 The stabilizer group within $Q^{6} \quad 24$

5.2 Further symmetries of $\Psi$ : lifting the hexacode automorphisms 25

5.3 The stabilizer group of $\operatorname{Im}(P)$ within $\mathrm{SU}(2)^{6} \quad 27$

5.4 Extending the automorphism groups to include left- and right-movers 28 5.4.1 Stabilizer of $(4,4)$ supersymmetry within $\operatorname{Spin}(4)^{6}: S_{6} / Z_{\text {diag }} \quad 28$

5.4.2 Stabilizer of $(4,1)$ supersymmetry within $\operatorname{Spin}(4)^{6}: S_{6} / Z_{\operatorname{diag}} \quad 34$

5.5 The "new" twined elliptic genera 34

5.6 Stabilizer of an $N=1$ supercurrent within a group that includes parity$\begin{array}{ll}\text { reversing operations } & 37\end{array}$

6 RR states and the MOG construction of the Golay code 38

7 Superconformal symmetry and QEC in Conway moonshine 40

7.1 Relation between conway and GTVW superconformal currents 43

A The automorphism group of the hexacode and the even Golay code $\quad 44$

$\begin{array}{ll}\text { B Supersymmetry conventions } & 47\end{array}$

$\begin{array}{ll}\text { B.1 Superconformal algebras } & 47\end{array}$ 

D Quaternions give a distinguished basis for the $(2 ; 2)$ representation of $\mathrm{SU}(2) \times \mathrm{SU}(2)$

\section{Introduction}

Physicists who wonder about the ultimate structure of our universe can be divided into two classes. The first class believes that our universe is chosen at random from a huge ensemble of possible universes. The second class believes that our universe and the fundamental laws that govern it are based on some beautiful and exceptional mathematical structure. String theory provides evidence for both points of view. Anomaly cancellation [51], the discovery of the heterotic string [53-55] and the discovery of Calabi-Yau compatifications [10] of string theory certainly involved beautiful and exceptional structures and connections between mathematical objects and the demands of physical consistency. Subsequent developments involving new constructions of Calabi-Yau spaces, the understanding of D-branes in string theory [90] and the multitudinous possibilities of (alleged) flux compactifications [26] have led to the idea of a huge landscape of string vacua and no clear principle that would select one special point in this landscape as describing our universe. The authors of the present paper are philosophically inclined towards the point of view of the second class of physicists and are thus interested in studying special points in the landscape of string compactifications that involve beautiful and exceptional mathematical structures. Certainly among these special points the ones associated to moonshine are amongst the most beautiful. The $c=24$ CFT underlying Monstrous Moonshine can be used to describe a compactification of the bosonic string to two dimensions. Similarly, the $c=12$ SCFT with Conway symmetry can be used to describe compactification of type II superstring theory to two dimensions. Moreover, the holomorphic $c=24$ Monster CFT and an anti-holomorphic version of the $c=12$ Conway theory can be used to describe a compactification of the heterotic string to two dimensions. Of course two dimensions is not a good description of the real world. More recently, moonshine phenomenon that are less well understood but closer to physical reality have been observed in the study of the elliptic genus of $K 3$ sigma models [35]. These sigma models provide a compactification of superstring theory to six spacetime dimensions and play a central role in various string dualities in four, five and six spacetime dimensions. Despite ten years of persistent effort by a small but devoted community of physicists and mathematicians the Mathieu (and Umbral) moonshine phenomena remain mysterious, hinting at an important gap in our understanding of symmetries of conformal field theories and string models.

Our motivations for the investigations in this paper were multifold. We wanted to understand the origin of moonshine for the sporadic group $M_{24}$ in the elliptic genus of $K 3$ and hoped that study of symmetries preserving $(4,1)$ superconformal symmetry might shed some light on this puzzle. We were also intrigued by our realization that error correcting 
codes appear naturally in the analysis of the special K3 sigma model discussed in [41]. We will henceforth refer to this model as the GTVW model in recognition of the analysis in [41] but elements of the model were actually constructed and discussed earlier [85, 97, 98]. As will become clear in later sections, we still do not understand the origin of $M_{24}$ but we were able to enlarge the possible symmetry transformations that can act on the $K 3$ elliptic genus beyond those previously considered.

Connections between classical error correcting codes and CFT have a long history. Many special lattices can be constructed from classical error correcting codes [18] and of course from a lattice one can construct a lattice VOA leading to a connection between special codes and special VOAs, see for example [23, 49]. There are also code VOAs which utilize classical codes to embed nontrivial CFTs into tensor products of simpler CFTs $[25,69,77,83]$. However as far as we are aware the role we have found for quantum error correcting codes in understanding the structure of superconformal field theories is new. Our study of codes in the GTVW model provides a nice example where both classical and quantum error correcting codes play important roles. In particular the classical hexacode plays a central role in understanding the symmetries of that model while the construction of superconformal generators in both the GTVW model and the $c=12$ Conway Moonshine CFT are linked to special quantum error correcting codes. We suspect that there is also such a connection for the superconformal generator in the version of the Monster CFT studied in [21] but there are many subtleties in the analysis which have kept us from presenting such an analysis here.

Throughout the text we indicate several directions where further research might be informative and useful. In addition to these, our work clearly raises the interesting question of what is the moduli space of $(4,1)$ sigma models smoothly connected to $(4,4) \mathrm{K} 3$ sigma models. In particular, one would like to know if there is an analog of the Quantum Mukai Theorem of Gaberdiel, Hohenegger, and Volpato in this context. For some literature on $(4,1)$ sigma models see [62-65]. In [67] $N=1$ superconformal structures on a large class of VOA's were classified. If the main message of this paper is correct, there should be connections to quantum error-correcting codes in these examples. It will be interesting to see if that turns out to be the case.

The outline of this paper is as follows. Following this introduction, the second section collects a number of useful facts regarding the field $\mathbb{F}_{4}$, the hexacode and the relation of these to the Golay code via the Miracle Octad Generator (MOG). We also briefly discuss quantum error correcting codes and highlight a particular one Qbit quantum error correcting code that will play a role in our later analysis. In section three we present one of our main results. We recall the GTVW model, and explain that the supercurrents in this model are related to quantum codes. Section four reviews some aspects of Mathieu moonshine and explains the relevance to this paper. The Mathieu moonshine observation regarding the sporadic group $M_{24}$ and the elliptic genus of K3 sigma models is based on a decomposition of the elliptic genus into characters of the $N=4$ superconformal algebra [35]. We point out that this decomposition depends only on the existence of $(4,1)$ superconformal symmetry and that the group of symmetry transformations preserving $(4,1)$ superconformal symmetry is in general larger than the possible groups of $(4,4)$ preserving transformations 
classified in [40]. Section five uses the relation of the supercurrents to codes to study the symmetry groups of the GTVW model that preserve various superconformal structures. We focus on the particular K3 sigma model of [41] and find a role for the Golay code in this model, namely there is an isomorphism between the Golay code and a certain stabilizer group of a left-right symmetric $N=1$ supercharge. Four appendices summarize background material, supersymmetry conventions and some technical details.

For some reviews of moonshine we can recommend $[32,46]$ for mathematically inclined readers and $[15,70]$ for physicists.

\section{Acknowledgments}

We thank D. Allcock, A. Banerjee, D. Freed, I. Frenkel, D. Harlow, T. Johnson-Freyd, C. Hull, T. Mainiero, D. Morrison, S.-H. Shao, A. Taormina and K. Wendland for helpful conversations and correspondence. We especially thank M. Gaberdiel, T. Johnson-Freyd, A. Taormina, and K. Wendland for numerous useful and important remarks on the draft. We gratefully acknowledge the hospitality of the Aspen Center for Physics (under NSF Grant No. PHY-1066293) where this work was initiated. Part of this work was also done at the Stanford Institute for Theoretical Physics, and GM would like to thank the SITP, and Shamit Kachru in particular, for hospitality. We have made use of the GAP package for finite group theory in our analysis [47] as well as the Magma Computer Algebra system which was made available through a grant from the Simons Foundation. JH acknowledges support from the $\mathrm{NSF}^{1}$ under grant PHY 1520748 and from the Simons Foundation (\#399639). GM is supported by the DOE under grant DOE-SC0010008 to Rutgers.

\section{Codes and error correction}

Codes, both classical and quantum, play a central role in our analysis. In this section we give the basic definitions of classical and quantum error correcting codes and provide examples that will play a role in our later analysis. Useful references include [7, 18, 50, 81, 91].

Let $q$ be a prime power. A classical q-ary code $\mathcal{C}$ of length $n$ is a set of vectors in the vector space $\mathbb{F}_{q}^{n}$. The vectors in the set $\mathcal{C}$ are called codewords: they are words with $n$ letters drawn from the finite field $\mathbb{F}_{q}$, regarded as an alphabet. If $\mathcal{C} \subset \mathbb{F}_{q}^{n}$ is a linear subspace it is called a linear code. The main examples we encounter in this paper are linear codes with $q=2$ and $q=4$. The dimension $k$ of a linear code is the dimension of the linear subspace spanned by the codewords. Such codes are said to be of type $[n, k]$. A code of type $[n, k]$ can be specified by a generator matrix $G$ which is a $k \times n$ matrix such that the code $\mathcal{C}$ is spanned by the rows of $G$ with coefficients in $\mathbb{F}_{q}$. One can always find a code equivalent to $\mathcal{C}$ such that the generator matrix takes the form

$$
G=\left[I_{k}, A\right]
$$

with $A$ a $k \times(n-k)$ matrix and $I_{k}$ the $k \times k$ identity matrix. We use the convention that code words $c$ (of length $n$ ) encode the message $m$ (of length $k$ ) as

$$
c=m G
$$

\footnotetext{
${ }^{1}$ Any opinions, findings, and conclusions or recommendations expressed in this material are those of the author(s) and do not necessarily reflect the views of the National Science Foundation.
} 
so that with $G$ in canonical form the first $k$ letters of $c$ are simply the elements of the message word $m$.

In the field $\mathbb{F}_{q}$ one has the conjugation (Frobenius) automorphism $x \rightarrow \bar{x}=x^{p}$ for $q=p^{\alpha}$. The dual or orthogonal code to a code $\mathcal{C}$ is defined to be

$$
\mathcal{C}^{\perp}=\left\{x \in \mathbb{F}_{q}^{n}: x \cdot \bar{u}=0, \text { all } u \in \mathcal{C}\right\}
$$

The dimension of $\mathcal{C}^{\perp}$ is $n-\operatorname{dim} \mathcal{C}$. The code is called self-dual if $\mathcal{C}=\mathcal{C}^{\perp}$. A useful fact is that if $\mathcal{C}$ has generator matrix given by eq. (2.1) then a generator matrix for $\mathcal{C}^{\perp}$ is

$$
H=\left[-\bar{A}^{t r}, I_{n-k}\right] .
$$

$H$ is also called the parity check matrix of the code $\mathcal{C}$.

The (Hamming) distance $d\left(c_{1}, c_{2}\right)$ between any two code words $c_{1}, c_{2}$ is the number of places at which $c_{1}$ and $c_{2}$ differ. The Hamming distance of a code is the minimum distance between any two codewords,

$$
d(\mathcal{C})=\min _{c_{1}, c_{2} \in \mathcal{C}, c_{1} \neq c_{2}} d\left(c_{1}, c_{2}\right)
$$

The Hamming distance is often included in the specification of a code by writing $[n, k, d]$ for a $[n, k]$ code with Hamming distance $d$.

We now give a number of examples of codes that play a role in our later analysis.

\subsection{Codes related to the Hamming code}

The codes which govern the $N=1$ superconformal symmetries in a K3 sigma model we study below are closely related to the renowned Hamming code.

The Hamming code is a binary linear $[7,4,3]$ code over $\mathbb{F}_{2}$ with generator matrix.

$$
G=\left(\begin{array}{lllllll}
1 & 0 & 0 & 0 & 1 & 1 & 0 \\
0 & 1 & 0 & 0 & 1 & 0 & 1 \\
0 & 0 & 1 & 0 & 0 & 1 & 1 \\
0 & 0 & 0 & 1 & 1 & 1 & 1
\end{array}\right)
$$

The Hamming code can famously detect and correct any single bit error. Adding a parity bit to the code gives the $[8,4]$ Hamming code which can also detect (but not correct) errors in two bits.

If we simply drop the seventh bit of the Hamming code we obtain instead a $[6,4]$ binary linear code. Relabelling the entries as $\left\{x_{1}+x_{2}+x_{4}, x_{2}, x_{1}+x_{3}+x_{4}, x_{3}, x_{4}, x_{1}\right\}$ we obtain a code $\mathcal{S}_{N=1}$ of type $[6,4]$ whose sixteen codewords can be listed as:

$$
\begin{gathered}
,[123456],[1234],[3456],[1256],[12],[34],[56], \\
{[135],[245],[236],[146],[246],[235],[136],[145] .}
\end{gathered}
$$

Here we are using a notation where we list only the entries that are 1 for each word. We have adopted this specific choice in order to facilitate comparison to later expressions. See e.g. equation (3.9). 
A subcode $\mathcal{S}_{N=4}$ of this truncated Hamming code will also play a role in describing $N=4$ supercurrents. $\mathcal{S}_{N=4}$ is a code of type [6,3] spanned by

$$
\begin{aligned}
& w_{1}=(0,0,1,1,1,1):=[3456] \\
& w_{2}=(0,1,0,1,0,1):=[246] \\
& w_{3}=(0,0,0,0,1,1):=[56]
\end{aligned}
$$

\subsection{The hexacode}

The hexacode utilizes the field $\mathbb{F}_{4}$. Since some properties of this field might not be familiar to physicists we briefly review them here. We think of it concretely as the set

$$
\mathbb{F}_{4}=\{\underline{0}, \underline{1}, \underline{\omega}, \underline{\bar{\omega}}\} .
$$

To define the Abelian group law for addition of vectors we take $\underline{0}$ to be the additive identity and

$$
\begin{aligned}
& \underline{1}+\underline{\omega}=\underline{\bar{\omega}} \\
& \underline{1}+\underline{\bar{\omega}}=\underline{\omega} \\
& \underline{\omega}+\underline{\bar{\omega}}=\underline{1} \\
& x+x=\underline{0} .
\end{aligned}
$$

We write $\mathbb{F}_{4}^{+}$for $\mathbb{F}_{4}$ considered as an Abelian group with the + law. As an Abelian group it is isomorphic to $\mathbb{Z}_{2} \oplus \mathbb{Z}_{2}$. Note that $\mathbb{F}_{4}$ is a field of characteristic two, so $2 x=\underline{0}$ for all $x$, and there is no distinction between $x$ and $-x$.

The multiplicative Abelian group law for nonzero vectors $\mathbb{F}_{4}^{*}$ is defined by taking 1 to be the multiplicative identity and

$$
\begin{aligned}
& \underline{\omega \omega}=\underline{\bar{\omega}} \\
& \underline{\omega \bar{\omega}}=\underline{1} \\
& \underline{\bar{\omega} \bar{\omega}}=\underline{\omega}
\end{aligned}
$$

Recall that $\mathbb{F}_{4}$ has a $\mathbb{Z}_{2}$ (Frobenius) automorphism $x \rightarrow x^{2}$. This automorphism preserves the additive and multiplicative identities $\underline{0}$ and $\underline{1}$ and takes takes

$$
\underline{\omega} \leftrightarrow \underline{\bar{\omega}} .
$$

In our analysis of the GTVW K3 sigma model an important role is played by a group homomorphism from the quaternion subgroup of $\mathrm{SU}(2)$ to $\mathbb{F}_{4}^{+}$. To define this we first consider the quaternion group $Q \subset \mathrm{SU}(2)$ generated by $\mathrm{i} \sigma^{1}, \mathrm{i} \sigma^{2}, \mathrm{i} \sigma^{3}$ where the $\sigma^{i}$ are the standard Pauli matrices. This is an 8 element group. Explicitly:

$$
Q=\left\{ \pm 1, \pm \mathrm{i} \sigma^{1}, \pm \mathrm{i} \sigma^{2}, \pm \mathrm{i} \sigma^{3}\right\}
$$

with group composition given by matrix multiplication. There is a homomorphism to $\mathbb{F}_{4}^{+}$ with kernel given by the subgroup $\{ \pm 1\}$ :

$$
1 \rightarrow\{ \pm 1\} \rightarrow Q \stackrel{\pi}{\rightarrow} \mathbb{F}_{4}^{+} \rightarrow 1
$$


The sequence does not split. We will make a specific choice of section $h$ of $\pi$ in our computations:

$$
\begin{aligned}
h(\underline{0}) & =\left(\begin{array}{ll}
1 & 0 \\
0 & 1
\end{array}\right) \\
h(\underline{1}) & =\left(\begin{array}{cc}
0 & 1 \\
-1 & 0
\end{array}\right)=i \sigma^{2} \\
h(\underline{\omega}) & =\left(\begin{array}{ll}
0 & \mathrm{i} \\
\mathrm{i} & 0
\end{array}\right)=i \sigma^{1} \\
h(\underline{\bar{\omega}}) & =\left(\begin{array}{cc}
-\mathrm{i} & 0 \\
0 & \mathrm{i}
\end{array}\right)=-i \sigma^{3} .
\end{aligned}
$$

There is also a relation of the multiplicative structure of $\mathbb{F}_{4}$ with the quaternion group:

$$
\begin{aligned}
& h(\underline{\omega} x)=h(x \underline{\omega})=\Omega^{-1} h(x) \Omega \\
& h(\underline{\bar{\omega}} x)=h(x \underline{\bar{\omega}})=\Omega^{-2} h(x) \Omega^{2}=\Omega h(x) \Omega^{-1} .
\end{aligned}
$$

Note the second equation immediately follows from the first since

$$
h(\underline{\bar{\omega}} x)=h(\underline{\omega \omega} x)=\Omega^{-1} h(\underline{\omega} x) \Omega=\Omega^{-2} h(x) \Omega^{2}
$$

In the above

$$
\Omega=\left(\begin{array}{cc}
\frac{1}{2}(1-\mathrm{i}) & \frac{1}{2}(1+\mathrm{i}) \\
-\frac{1}{2}(1-\mathrm{i}) & \frac{1}{2}(1+\mathrm{i})
\end{array}\right)=\frac{1}{\sqrt{2}}\left(\begin{array}{cc}
1 & 1 \\
-1 & 1
\end{array}\right)\left(\begin{array}{cc}
e^{-\mathrm{i} \pi / 4} & 0 \\
0 & e^{\mathrm{i} \pi / 4}
\end{array}\right)
$$

We collect here a few useful properties of $\Omega$

1. $\Omega^{3}=-1$ so $\Omega^{-1}=-\Omega^{2}$.

2. $\Omega$ is an $\mathrm{SU}(2)$ matrix. Its action on $\mathbb{R}^{3}$ is an order three rotation around the axis through $(1,1,1)$ so it permutes $x \rightarrow y \rightarrow z \rightarrow x$. This mirrors the action of multiplication by $\omega$ which permutes the 3 elements of $\mathbb{F}_{4}^{*}$ :

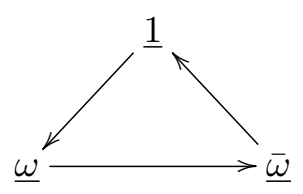

Returning to (2.15), we can define a $\mathbb{Z}_{2}$-valued cocycle

$$
h(x) h(y)=\epsilon(x, y) h(x+y)
$$

for $x, y \in \mathbb{F}_{4}$. This cocycle has some nice properties:

1. It is 1 if $x$ or $y$ is the additive identity,

$$
\epsilon(0, x)=\epsilon(x, 0)=1
$$


2. The diagonal values are

$$
\epsilon(x, x)= \begin{cases}+1 & x=\underline{0} \\ -1 & x \neq \underline{0} .\end{cases}
$$

3. $\epsilon$ is "permutation invariant"

$$
\epsilon(\underline{\omega} x, \underline{\omega} y)=\epsilon(x, y) \text {. }
$$

4. $\epsilon$ is bimultiplicative:

$$
\begin{aligned}
& \epsilon\left(x_{1}+x_{2}, y\right)=\epsilon\left(x_{1}, y\right) \epsilon\left(x_{2}, y\right), \\
& \epsilon\left(x, y_{1}+y_{2}\right)=\epsilon\left(x, y_{1}\right) \epsilon\left(x, y_{2}\right) .
\end{aligned}
$$

5. For $x, y \neq \underline{0}$ we have, from $(2.15)$,

$$
\epsilon_{x, y}=\left(\begin{array}{ccc}
-1 & -1 & 1 \\
1 & -1 & -1 \\
-1 & 1 & -1
\end{array}\right)
$$

To prove permutation invariance (2.23) we simply conjugate (2.20) by $\Omega$ and use (2.16). To prove the bimultiplicative property we first note that it is obvious if any argument is zero, so we can assume all arguments are nonzero. We are not aware of any better proof than an explicit check of the 9 cases to estabish $\epsilon\left(x_{1}+x_{2}, y\right)=\epsilon\left(x_{1}, y\right) \epsilon\left(x_{2}, y\right)$. Then one uses the symmetry properties of $\epsilon$.

We now turn to a discussion of the hexacode $\mathcal{H}_{6}$, a three-dimensional subspace of $\mathbb{F}_{4}^{6}$ consisting of codewords $\left(x_{1}, \ldots, x_{6}\right)$ such that, if

$$
\Phi_{x_{1}, x_{2}, x_{3}}(y):=x_{1} y^{2}+x_{2} y+x_{3}
$$

then

$$
\begin{aligned}
& x_{4}=\Phi_{x_{1}, x_{2}, x_{3}}(\underline{1}) \\
& x_{5}=\Phi_{x_{1}, x_{2}, x_{3}}(\underline{\omega}) \\
& x_{6}=\Phi_{x_{1}, x_{2}, x_{3}}(\underline{\bar{\omega}})
\end{aligned}
$$

A full list of the 64 hexacode words (which can occasionally be quite helpful when doing computations) is shown in appendix A where we also analyze the automorphism group of the hexacode.

Note that $\Phi_{x_{1}, x_{2}, x_{3}}$ depends linearly on $x_{1}, x_{2}, x_{3}$ so this makes manifest that we have a three-dimensional subspace. In fact, taking $\left(x_{1}, x_{2}, x_{3}\right)=(\underline{1}, \underline{0}, \underline{0}),(\underline{0}, \underline{1}, \underline{0}),(\underline{0}, \underline{0}, \underline{1})$ we automatically get a basis

$$
\begin{aligned}
& b_{1}=(\underline{1}, \underline{0}, \underline{0}, \underline{1}, \underline{\bar{\omega}}, \underline{\omega}) \\
& b_{2}=(\underline{0}, \underline{1}, \underline{0}, \underline{1}, \underline{\omega}, \underline{\bar{\omega}}) \\
& b_{3}=(\underline{0}, \underline{0}, \underline{1}, \underline{1}, \underline{1}, \underline{1})
\end{aligned}
$$


corresponding to the generator matrix

$$
\left(\begin{array}{llllll}
\underline{1} & \underline{0} & \underline{0} & \underline{1} & \underline{\bar{\omega}} & \underline{\omega} \\
\underline{0} & \underline{1} & \underline{0} & \underline{1} & \underline{\omega} & \underline{\bar{\omega}} \\
\underline{0} & \underline{0} & \underline{1} & \underline{1} & \underline{1} & \underline{1}
\end{array}\right)=(1, A)
$$

We can make an inner product space with a nondegenerate $\mathbb{F}_{4}$-valued inner product:

$$
\langle x, y\rangle:=\sum_{i=1}^{6} \bar{x}_{i} y_{i}
$$

There is nice relationship between the hexacode and the $[6,4]$ binary linear code defined earlier. To see this we first define a homomorphism of Abelian groups

$$
\pi: \mathbb{F}_{4}^{+} \rightarrow \mathbb{F}_{2}
$$

such that

$$
\begin{array}{ll}
\pi(\underline{0})=0 & \pi(\underline{\bar{\omega}})=0 \\
\pi(\underline{1})=1 & \pi(\underline{\omega})=1 .
\end{array}
$$

The conceptual reason behind this choice of $\pi$ is related to the quantum code interpretation discussed below: operators $h(x)$ that do not induce a bit-flip map to 0 and those that induce a bit-flip map to 1 . We can extend this in an obvious way to

$$
\pi:\left(\mathbb{F}_{4}^{+}\right)^{6} \rightarrow \mathbb{F}_{2}^{6} .
$$

Applying $\pi$ to the 64 code words of the hexacode clearly produces the 16 words of a binary linear $[6,4]$ code and a short computation shows that the resulting $[6,4]$ code is isomorphic to the one defined in equation (2.7).

The dual, or orthogonal, code $\mathcal{H}_{6}^{*}$ is defined by the set of $x$ so that $\langle y, x\rangle=0$ for all $y \in \mathcal{H}_{6}$. It is not hard to check that the hexacode is self-dual: it is maximal isotropic.

It is useful to note another maximal isotropic subspace $\mathcal{H}_{6}^{\perp}$ so that we have a decomposition into maximal isotropic subspaces:

$$
\mathbb{F}_{4}^{6} \cong \mathcal{H}_{6} \oplus \mathcal{H}_{6}^{\text {comp }}
$$

We can do this as follows. Let $e_{i}$ be the vector with a $\underline{1}$ in the $i^{\text {th }}$ coordinate and $\underline{0}$ elsewhere. Clearly we have

$$
\left\langle e_{i}, b_{j}\right\rangle=\delta_{i j} \quad 1 \leq i, j \leq 3
$$

But the $e_{i}$ do not span an isotropic subspace. Nevertheless, since the hexacode is isotropic we can try to modify the span of $e_{i}, i=1,2,3$ to the span of

$$
u_{i}=e_{i}+A_{i j} b_{j}
$$

and then the desired

$$
\left\langle u_{i}, u_{j}\right\rangle=\underline{0}
$$


implies that

$$
\delta_{i j}+\bar{A}_{i j}+A_{j i}=\underline{0}
$$

There are many solutions to this, but a simple one is to take $A_{i j}=\underline{\omega} \delta_{i j}$. So if

$$
\begin{aligned}
& u_{1}=(\underline{\bar{\omega}}, \underline{0}, \underline{0}, \underline{\omega}, \underline{1}, \underline{\bar{\omega}}) \\
& u_{2}=(\underline{0}, \underline{\bar{\omega}}, \underline{0}, \underline{\omega}, \underline{\bar{\omega}}, \underline{1}) \\
& u_{3}=(\underline{0}, \underline{0}, \underline{\bar{\omega}}, \underline{\omega}, \underline{\omega}, \underline{\omega})
\end{aligned}
$$

then

$$
\left\langle b_{i}, u_{j}\right\rangle=\delta_{i j} \quad\left\langle u_{i}, u_{j}\right\rangle=0
$$

so the span of $u_{i}$ will serve as a maximal isotropic complementary space $\mathcal{H}_{6}^{\text {comp }}$.

\subsection{The hexacode and the Golay code}

In this section we rephrase slightly the description of the "Miracle Octad Generator" (MOG) [20] which can be found in [18]. This is a particularly efficient way of thinking about the Golay code. In [41] the MOG was connected to the space of R-R ground states of a particular K3 sigma model and our later analysis will elaborate on, and clarify, this connection.

The MOG is based on a map from digits in $\mathbb{F}_{4}$ to $\mathbb{F}_{2}^{4}$. There are four "interpretations" of the digits and these can be summarized by a pair of maps

$$
g^{ \pm}: \mathbb{F}_{4} \times \mathbb{F}_{2} \rightarrow \mathbb{F}_{2}^{4}
$$

The map $g^{+}$corresponds to what SPLAG [18] refers to as "even interpretation of hexacode digits" and is defined by:

$$
\begin{aligned}
& g^{+}(\underline{0}, 0)=\left(\begin{array}{l}
0 \\
0 \\
0 \\
0
\end{array}\right) \quad g^{+}(\underline{0}, 1)=\left(\begin{array}{l}
1 \\
1 \\
1 \\
1
\end{array}\right) \quad g^{+}(\underline{1}, 0)=\left(\begin{array}{l}
0 \\
0 \\
1 \\
1
\end{array}\right) \quad g^{+}(\underline{1}, 1)=\left(\begin{array}{l}
1 \\
1 \\
0 \\
0
\end{array}\right) \\
& g^{+}(\underline{\omega}, 0)=\left(\begin{array}{l}
0 \\
1 \\
0 \\
1
\end{array}\right) \quad g^{+}(\underline{\omega}, 1)=\left(\begin{array}{l}
1 \\
0 \\
1 \\
0
\end{array}\right) \quad g^{+}(\underline{\bar{\omega}}, 0)=\left(\begin{array}{l}
0 \\
1 \\
1 \\
0
\end{array}\right) \quad g^{+}(\underline{\underline{\omega}}, 1)=\left(\begin{array}{l}
1 \\
0 \\
0 \\
1
\end{array}\right)
\end{aligned}
$$

Note that $g^{+}$is in fact a group homomorphism $\mathbb{F}_{4}^{+} \times \mathbb{F}_{2} \rightarrow \mathbb{F}_{2}^{4}$.

Now we define a group homomorphism

$$
\mathfrak{f}^{+}:\left(\mathbb{F}_{4}^{+} \times \mathbb{F}_{2}\right)^{6} \rightarrow \mathbb{F}_{2}^{24}
$$

It is very useful to represent the vectors in $\mathbb{F}_{2}^{24}$ as a $4 \times 6$ array of elements of $\mathbb{F}_{2}$. Each column is the result of $g^{+}$applied to a "decorated digit" in $\mathbb{F}_{4} \times \mathbb{F}_{2}$. That is,

$$
\mathfrak{f}^{+}\left(x_{1}, \epsilon_{1}, \ldots, x_{6}, \epsilon_{6}\right)=\left(g^{+}\left(x_{1}, \epsilon_{1}\right), \ldots, g^{+}\left(x_{6}, \epsilon_{6}\right)\right) \quad x_{\alpha} \in \mathbb{F}_{4} \quad \epsilon_{\alpha} \in \mathbb{F}_{2}
$$




\begin{tabular}{|c|c|c|c|c|c|}
\hline 0 & 1 & 0 & 1 & 1 & 1 \\
0 & 1 & 0 & 1 & 0 & 0 \\
0 & 0 & 0 & 0 & 1 & 0 \\
0 & 0 & 0 & 0 & 0 & 1 \\
\hline$\underline{0}$ & $\underline{1}$ & $\underline{0}$ & $\underline{1}$ & $\underline{\omega}$ & $\underline{\bar{\omega}}$ \\
\hline
\end{tabular}

Table 1. Illustration of the MOG construction of a Golay code word from an even interpretation of the hexacode word $0101 \omega \bar{\omega}$ which is displayed along the bottom of the table.

See table 1 for an illustration of this construction. We will refer to elements

$$
\left(x_{1}, \epsilon_{1}, \ldots, x_{6}, \epsilon_{6}\right) \in\left(\mathbb{F}_{4} \times \mathbb{F}_{2}\right)^{6}
$$

as decorated words, viewing $w=\left(x_{1}, \ldots, x_{6}\right) \in \mathbb{F}_{4}^{6}$ as the word and $\left(\epsilon_{1}, \ldots, \epsilon_{6}\right)$ as the decorations. We also denote decorated words as $(x, \epsilon)$.

The first nontrivial fact is that the image of $\mathfrak{f}^{+}$on the decorated words $(x, \epsilon)$ with

$$
x \in \mathcal{H}_{6} \quad \sum_{\alpha=1}^{6} \epsilon_{\alpha}=0 \bmod 2
$$

is an index two subspace of the Golay code $\mathcal{G} \subset \mathbb{F}_{2}^{24}$. We will call it the even Golay code and denote it by $\mathcal{G}^{+}$. This is a set of $2^{11}$ vectors and forms a subgroup of the Golay code because $g^{+}$and $\mathfrak{f}^{+}$are homomorphisms.

The complement of the set of even Golay code words inside the set of all Golay code words is the set of odd Golay code words. A good way to parametrize the odd Golay code words is to introduce the column vector

$$
p=\left(\begin{array}{l}
1 \\
0 \\
0 \\
0
\end{array}\right)
$$

and we then define

$$
g^{-}(x, \epsilon):=g^{+}(x, \epsilon)+p
$$

So

$$
\begin{array}{ll}
g^{-}(\underline{0}, 0)=\left(\begin{array}{l}
1 \\
0 \\
0 \\
0
\end{array}\right) & g^{-}(\underline{0}, 1)=\left(\begin{array}{l}
0 \\
1 \\
1 \\
1
\end{array}\right) \\
g^{-}(\underline{\omega}, 0)=\left(\begin{array}{l}
1 \\
1 \\
0 \\
1
\end{array}\right) & g^{-}(\underline{\underline{1}}, 0)=\left(\begin{array}{l}
1 \\
0 \\
1 \\
1
\end{array}\right) \quad g^{-}(\underline{1}, 1)=\left(\begin{array}{l}
0 \\
1 \\
0 \\
0
\end{array}\right)
\end{array}
$$


Now, the odd Golay code words are

$$
w=\left(g^{-}\left(x_{1}, \epsilon_{1}\right), \ldots, g^{-}\left(x_{6}, \epsilon_{6}\right)\right)
$$

with

$$
\sum_{\alpha=1}^{6} \epsilon_{\alpha}=1 \bmod 2 .
$$

The MOG construction of the Golay code gives a nice connection between the automorphism group of the even Golay code $\mathcal{G}^{+}$and the holomorph of the hexacode. (See appendix A for the definition of the holomorph of a group.) The hexacode itself is $2^{6}$ as an Abelian group and its automorphism group is described in appendix A. The holomorph has the structure $2^{6}: 3 . S_{6}$. The hexacode acts by

$$
w \cdot \mathfrak{f}^{+}(x, \epsilon)=\mathfrak{f}^{+}(x+w, \epsilon)
$$

for $w \in \mathcal{H}_{6}$ and

$$
\varphi \cdot \mathfrak{f}^{+}(x, \epsilon)=\mathfrak{f}^{+}\left(\varphi^{-1}(x),(p(\varphi))^{-1} \cdot \epsilon\right)
$$

where $\varphi \in \operatorname{Aut}\left(\mathcal{H}_{6}\right)$ and $p$ is defined in equation (A.10). The automorphism group of $\mathcal{G}^{+}$within $S_{24}$ can be shown to be exactly the maximal subgroup of $M_{24}$ known as the sextet group. ${ }^{2}$ The sextet group is nicely described in [18] (see also the very informative Wikipedia article on $M_{24}$ ). In fact the sextet group is exactly the same as the holomorph of the hexacode.

\subsection{Quantum codes}

Quantum error correcting (QEC) codes are designed to detect and correct errors in the transmission and processing of quantum information. The set of error operations $\mathcal{E}$ is a subset of the space of completely positive, non-trace increasing maps on the space of density matrices. We define a QEC as follows:

Definition. Let $H$ be a Hilbert space. A Hilbert subspace $C \subset H$ is an error correcting quantum code with respect to a set $\mathcal{E}$ of error operations if, for every $E_{i} \in \mathcal{E}$, we have

$$
P E_{i}^{\dagger} E_{j} P=\alpha_{i j} P
$$

where $P$ is the projector onto $C$ and $\alpha_{i j}$ is a nonzero Hermitian matrix.

When the above condition is satisfied it is possible to construct a quantum operation $\mathcal{R}$ that detects and then corrects the quantum errors $E_{i}$ while preserving the quantum information carried by states in the quantum code $C$. In some cases we will consider codes that can only detect but not correct quantum errors.

The QECs we consider are constructed as subspaces of the $n$-Qbit Hilbert space $\mathcal{H}_{n}=$ $\left(\mathbb{C}^{2}\right)^{\otimes n}$ and the set of error operations will consist of tensor products of elements $X_{i}, Y_{i}, Z_{i}$ which act on the $i^{t h}$ component of $\mathcal{H}_{n}$ as the Pauli matrices

$$
X=\left(\begin{array}{ll}
0 & 1 \\
1 & 0
\end{array}\right), \quad Y=\left(\begin{array}{cc}
0 & -\mathrm{i} \\
\mathrm{i} & 0
\end{array}\right), \quad Z=\left(\begin{array}{cc}
1 & 0 \\
0 & -1
\end{array}\right)
$$

\footnotetext{
${ }^{2}$ We thank D. Allcock for explaining this to us.
} 
We refer to these tensor products $E_{a}$ as Pauli operators and we define the weight of a Pauli operator to be the number of entries in the tensor product that have a non-trivial Pauli operator $(X, Y, Z)$ rather than the identity operator $(I)$. Thus $Z \otimes X \otimes I \otimes I$ has weight two.

If the code subspace of dimension $2^{k}$ is embedded in $\mathcal{H}_{n}$ with $k<n$ we will refer to the code as a quantum code of type $[[n, k]]$, where the double square brackets are used to distinguish quantum codes from classical codes as in [91]. The code distance of a quantum code is the minimum weight of a Pauli operator in $\mathcal{E}$ that is not the identity operator on $C$. If a quantum code is of type $[[n, k]]$ and has code distance $d$ we say it is of type $[[n, k, d]]$.

Stabilizer QEC codes are an important class of quantum codes that have much in common with classical linear codes. In the stabilizer formalism one considers the Pauli group $G_{n}$ acting on $n$ Qbits. The Pauli group acting on a single Qbit has a matrix representation consisting of the Pauli matrices along with multiplicative factors of \pm 1 and \pm i. Explicitly

$$
G_{1}=\{ \pm I, \pm \mathrm{i} I, \pm X, \pm \mathrm{i} X, \pm Y, \pm \mathrm{i} Y, \pm Z, \pm \mathrm{i} Z\}
$$

with $I$ the two by two identity matrix. (The quaternion group $Q$ is the intersection $Q=$ $G_{1} \cap \mathrm{SU}(2)$ :

$$
Q=\{ \pm I, \pm \mathrm{i} X, \pm \mathrm{i} Y, \pm \mathrm{i} Z\}
$$

and will be of some use later.)

Elements of the general Pauli group $G_{n}$ are n-fold tensor products of elements of $G_{1}$. Let $S$ be a subgroup of $G_{n}$ and $V^{S}$ the subspace of $\mathcal{H}_{n}$ stabilized by $S$. (We have $V^{S}=\{0\}$ if $S$ contains the group element -1, so we avoid this case.) Many important quantum error correcting codes can be constructed as subspaces of the form $V^{S}$ for particular choices of $S$.

An important example is the smallest QEC capable of detecting and correcting an arbitrary single one Qbit error, that is a code with $\mathcal{E}=\{X, Y, Z\}$. This code is of type $[[5,1,3]]$ and can be viewed as a stabilizer code with stabilizer group $S$ generated by the four elements

$$
\begin{aligned}
& M_{1}=X Z Z X I \\
& M_{2}=I X Z Z X \\
& M_{3}=X I X Z Z \\
& M_{4}=Z X I X Z
\end{aligned}
$$

Here as is usual in the QEC literature $X Z Z X I$ is shorthand for $X \otimes Z \otimes Z \otimes X \otimes I$ acting on the 5-Qbit space $\left(\mathbb{C}^{2}\right)^{\otimes 5}$. Note that $M_{2,3,4}$ are obtained from $M_{1}$ by cyclic permutation. An orthonormal basis for the code subspace can be constructed by starting with $|00000\rangle$ or $|11111\rangle$ and summing over all elements of the stabilizer group $S$. This leads to the basis vectors (see e.g. section 10.5.6. of [88])

$$
\begin{aligned}
\left|0_{L}\right\rangle=\frac{1}{4}(|00000\rangle+|10010\rangle+|01001\rangle+|10100\rangle \\
+|01010\rangle-|11011\rangle-|00110\rangle-|11000\rangle \\
-|11101\rangle-|00011\rangle-|11110\rangle-|01111\rangle \\
-|10001\rangle-|01100\rangle-|10111\rangle+|00101\rangle)
\end{aligned}
$$


and $\left|1_{L}\right\rangle=X^{\otimes 5}\left|0_{L}\right\rangle$. There is also a $[[6,0,4]]$ code which is one-dimensional and is constructed from the basis of the $[[5,1,3]]$ code and an ancillary Qbit as

$$
\Psi_{[[6,0,4]]}=\frac{1}{\sqrt{2}}(|0\rangle \otimes|\overline{0}\rangle+|1\rangle \otimes|\overline{1}\rangle)
$$

Remark. J. Preskill [91] notes that this code state is maximally entangled in that the density matrix obtained by tracing over any 3 Qbits is totally random, $\rho^{(3)}=I / 8$. Strictly speaking this is an error detecting rather than error correcting code in that it can detect, but not correct, any single Qbit error. In [79] T. Mainiero defines several notions of "entanglement homology," a homological generalization of entanglement entropy which measures correlations between many body operators in a given state. The Poincaré polynomial for $\left|0_{L}\right\rangle$, which is a priori a five-term polynomial in $q$ turns out to be simply $51 q^{2}$ for Mainiero's "GNS cohomology," while the Poincaré polynomials for other related homologies are similarly quite simple. In other words: the state, while maximally entangled has unusual correlation properties.

\section{Supercurrents, states, and codes}

In this section we show that the spectra and supercurrents of several SCFTs that exhibit Moonshine phenomena can be rephrased in terms of both classical and quantum error correcting codes.

\subsection{The GTVW model}

The GTVW model is a $(4,4)$ sigma model with a very specific $T^{4} / \mathbb{Z}_{2}$ target [41]. It is a distinguished $\mathrm{K} 3$ because it has the maximal group $2^{8}: M_{20}$ allowed by the results of [40] (GHV). (In section 5.4.1 we will describe the structure of the group in excruciating detail.) The target $T^{4}$ is the $\operatorname{Spin}(8)$ maximal torus. Reference [41] shows that there are several incarnations of this model and the one we find most convenient is an extension of a product of six SU(2) level 1 WZW models. (Equivalently, the product of six Gaussian models at the T-duality invariant point $R=1$.)

Recall that the $\mathrm{SU}(2)$ level $1 \mathrm{WZW}$ model has affine $\mathrm{SU}(2)_{L} \times \mathrm{SU}(2)_{R}$ symmetry with both factors at level 1 . The unitary highest weight representations of affine level $1 \mathrm{SU}(2)$ will be labeled by $\mathcal{V}_{0}$ and $\mathcal{V}_{1}$ according to whether the states of lowest $L_{0}$ value are in the singlet or doublet of the global SU(2) symmetry. The product of six such models therefore has a Hilbert space of states on the circle given by

$$
\mathcal{H}=\left(\mathcal{V}_{0} \otimes \tilde{\mathcal{V}}_{0} \oplus \mathcal{V}_{1} \otimes \tilde{\mathcal{V}}_{1}\right)^{\otimes 6}
$$

where a tilde denotes right-movers. In the GTVW model we take instead the Hilbert space to be

$$
\mathcal{H}^{\mathrm{GTVW}}=\oplus_{(s, \tilde{s}) \in \mathcal{S}} \mathcal{V}_{s} \otimes \tilde{\mathcal{V}}_{\tilde{s}}
$$

where $s, \tilde{s} \in \mathbb{F}_{2}^{6}$ and $\mathcal{S} \subset \mathbb{F}_{2}^{6} \times \mathbb{F}_{2}^{6}$ is the subgroup defined by demanding that the components of $s, \tilde{s}$ obey $s_{\alpha}=\tilde{s}_{\alpha}+x$ for all $1 \leq \alpha \leq 6$ where $x \in \mathbb{F}_{2}$ is independent of $\alpha$ and can be 
either 0 or 1 . One easily checks that the space of fields is closed under operator product expansion since the fusion rules for $\mathcal{V}_{0}, \mathcal{V}_{1}$ are simply addition in $\mathbb{F}_{2}$. However, the fields are only mutually local up to sign: the GTVW model is not a standard CFT but an extension with a super-chiral algebra (a super-vertex-operator algebra) in which the modules all have mutual locality at worst \pm 1 . As we will see, the theory of codes pervades this model, and it is worth noting that the spectrum itself can be described by a code of type $[12,7]$, although we will not make use of that fact.

It is useful to note that the space of states $(3.2)$ is $\left(\mathbb{F}_{2} \oplus \mathbb{F}_{2}\right)$-graded. One grading is provided by $x$ and the other is $y=\sum_{\alpha} s_{\alpha}$. In terms of the original supersymmetric K3 sigma model we can identify $\mathcal{H}^{\text {GTVW }}$ with the sum of NSNS and RR sectors of the sigma model. The quantum number $y=0,1$ distinguishes the NS-NS sector from the R-R sector, respectively. Moreover reference [41] shows that $e^{\mathrm{i} \pi x}$ can be identified with $(-1)^{F_{L}+F_{R}}$ where $F_{L, R}$ are left- and right-moving fermion numbers. Thus, the GSO-projected version of this model, that is, the GSO projected K3 sigma model is equivalent to the product of six $k=1 \mathrm{SU}(2)$ WZW models.

Remark. $\mathbb{Z}_{2}$-extensions of vertex operator algebras go back to the very beginning of superstring theory [87, 92], and play a crucial role in formulating tachyon-free models [48]. They have also played a role in previous investigations into Moonshine [21]. A modern interpretation of these " $\mathbb{Z}_{2}$ extensions" of a conformal field theory is that one is defining a theory that depends on both conformal structure and spin structure. The procedure is to identity a nonanomalous $\mathbb{Z}_{2}$ global symmetry of the bosonic CFT, couple to an invertible topological field theory sensitive to spin structure known as the Arf theory, and gauge the diagonal global $\mathbb{Z}_{2}$ symmetry of the product theory. The resulting theory has a superVOA as its "chiral algebra." The GSO projection of the theory reproduces the original bosonic CFT. ${ }^{3}$ In our case, the original bosonic CFT is the product of six SU(2) level $k=1$ WZW models, and the GTVW model is the spin extension based on the diagonal element $-1^{6} \in \mathrm{SU}(2)_{L}^{6}$ in the $\left(\mathrm{SU}(2)_{L} \times \mathrm{SU}(2)_{R}\right)^{6} \mathrm{WZW}$ model.

\section{$3.2(4,4)$ superconformal algebra in the GTVW model}

The K3 sigma model has $(4,4)$ supersymmetry. The left-moving $\mathrm{N}=4$ supercurrents can therefore be expressed in the WZW language. The currents must be (anti-) holomorphic primary fields of conformal dimension $3 / 2$. To give an explicit construction we use the Frenkel-Kac-Segal construction of level one affine $\mathrm{SU}(2)^{6}$ in terms of six free bosonic fields $X^{I}, I=1,2, \cdots 6$. We then note that for $\epsilon, \tilde{\epsilon} \in\{ \pm 1\}^{6}$ we define (anti-) holomorphic vertex operators of conformal dimension $6 \times \frac{1}{4}=\frac{3}{2}$.

$$
V_{\epsilon}=e^{\frac{\mathrm{i}}{\sqrt{2}} \epsilon \cdot X}(z) \quad \tilde{V}_{\tilde{\epsilon}}=e^{\frac{\mathrm{i}}{\sqrt{2}} \tilde{\epsilon} \cdot \tilde{X}}(\bar{z})
$$

(For simplicity we will drop cocycle factors as they will play no crucial direct role in our analysis below.) Any linear combination of these operators gives an (anti-) holomorphic

\footnotetext{
${ }^{3}$ This procedure, which in fact goes back to $[1,2]$ has also been used to formulate theories of self-dual fields $[8,100]$. It has recently been explored and extended further in [43, 73, 74, 78]. See the 2019 TASI lectures by Y. Tachikawa for a pedagogical introduction. We thank Shu-Heng Shao for guiding us through this recent literature.
} 
primary field so the space of holomorphic primary fields of dimension $3 / 2$ is a linear space of complex dimension $2^{6}$ and the $V_{\epsilon}$ provide a natural basis. We will find it useful to identify this vector space with the Hilbert space of six Qbits. The basis $V_{\epsilon}$ corresponds to the natural spin basis

$$
\left|\epsilon_{1}\right\rangle \otimes \cdots \otimes\left|\epsilon_{6}\right\rangle
$$

where, for a single tensor factor, $\{|\epsilon=+\rangle,|\epsilon=-\rangle\}$ is an ordered basis for a Qbit in which a basis of anti-Hermitian generators of $\mathfrak{s u}(2)$ has matrix representation $J^{i}=-\frac{i}{2} \sigma^{i}$, $i=1,2,3$. If $s \in\left(\mathbb{C}^{2}\right)^{\otimes 6}$ we can write $s=\sum_{\epsilon \in \mathbb{Z}_{2}^{6}} c(\epsilon)|\epsilon\rangle$ and then we define

$$
V_{s}=\sum_{\epsilon \in \mathbb{Z}_{2}^{6}} c(\epsilon) V_{\epsilon}
$$

to be the corresponding primary field.

The results of [41] are easily used to show that the $\mathrm{N}=4$ supercurrents of the $\mathrm{K} 3$ sigma model can be written as very special states in the six Qbit system. Using the conventions of appendix B we can express these currents as: ${ }^{4}$

$$
\begin{aligned}
Q^{1} & =\left(\frac{i-1}{2}\right) V_{\Psi^{1}} & \bar{Q}^{1} & =\left(\frac{i-1}{2}\right) V_{\bar{\Psi}^{1}} \\
Q^{2} & =\left(\frac{i-1}{2}\right) V_{\Psi^{2}} & \bar{Q}^{2} & =\left(\frac{i-1}{2}\right) V_{\bar{\Psi}^{2}}
\end{aligned}
$$

where

$$
\begin{aligned}
& \Psi^{1}=[\emptyset]+[3456]-\mathrm{i}[246]-\mathrm{i}[235]+\mathrm{i}[56]+\mathrm{i}[34]+[245]+[236] \\
& \Psi^{2}=-[1]-[13456]+\mathrm{i}[1246]+\mathrm{i}[1235]-\mathrm{i}[156]-\mathrm{i}[134]-[1245]-[1236] \\
& \bar{\Psi}^{1}=\mathrm{i}[123456]+[1234]+[1256]-\mathrm{i}[136]-\mathrm{i}[145]+[135]+[146]+\mathrm{i}[12] \\
& \bar{\Psi}^{2}=\mathrm{i}[23456]+[234]+[256]-\mathrm{i}[36]-\mathrm{i}[45]+[35]+[46]+\mathrm{i}[2]
\end{aligned}
$$

The notation here is the following: the integers denote the position of a down-spin in the tensor product of six up/down spins, all other spins being up. Thus, for example,

$$
\begin{aligned}
{[\emptyset] } & =|++++++\rangle \\
{[3456] } & =|++----\rangle \\
{[145] } & =|-++--+\rangle
\end{aligned}
$$

and so forth.

For reasons that will be explained later, we are particularly interested in automorphisms of the K3 sigma model preserving one holomorphic $\mathrm{N}=1$ supercurrent. If we impose a unitarity constraint then, up to a general $\mathrm{SU}(2)_{R}$ symmetry transformation the general $N=1$ current is proportional to $Q^{1}+\bar{Q}^{1}$, which in turn is proportional to $V_{\Psi}$ with

$$
\begin{aligned}
\Psi:= & {[\emptyset]+\mathrm{i}[123456]+([1234]+[3456]+[1256])+\mathrm{i}([12]+[56]+[34]) } \\
& +([135]+[245]+[236]+[146])-\mathrm{i}([246]+[235]+[136]+[145])
\end{aligned}
$$

We will show in the next section that this seemingly complicated state is in fact governed in a simple way by a quantum error detecting code on six Qbits.

\footnotetext{
${ }^{4}$ To compare to [41] we identify, $Q^{1}=G^{+}, \bar{Q}^{1}=G^{-}, \bar{Q}^{2}=G^{\prime}, Q^{2}=G^{\prime}$.
} 


\subsection{The hexacode representation of the $N=1$ supercurrent}

We are going to use the hexacode discussed in section 2.2 to construct a rank one projector $P$ on the Hilbert space of six Qbits. Then our state $\Psi$ will be a vector in the image of $P$.

The section $h: \mathbb{F}_{4} \rightarrow Q \subset \mathrm{SU}(2)$ defined in equation (2.15) can be extended to vectors in $\mathbb{F}_{4}^{6}$. If $w=\left(x_{1}, \ldots, x_{6}\right) \in \mathbb{F}_{4}^{6}$ then we define

$$
h(w):=h\left(x_{1}\right) \otimes h\left(x_{2}\right) \otimes h\left(x_{3}\right) \otimes h\left(x_{4}\right) \otimes h\left(x_{5}\right) \otimes h\left(x_{6}\right) \in \operatorname{End}\left(\left(\mathbb{C}^{2}\right)^{\otimes 6}\right)
$$

Important remark: an important point throughout this paper is that while the group $\mathrm{SU}(2)^{6}$ acts on the space of six Qbits, it does not act effectively. There is a subgroup

$$
Z \subset Z\left(\mathrm{SU}(2)^{6}\right)
$$

where $Z\left(\mathrm{SU}(2)^{6}\right) \cong \mathbb{Z}_{2}^{6}$ is the center, that acts ineffectively on six Qbits. It is the subgroup of six signs whose product is +1 . The group $Z$ is isomorphic to $\mathbb{Z}_{2}^{5}$. We will denote the quotient by $\mathrm{SU}(2)_{6}:=\mathrm{SU}(2)^{6} / Z$. (Some readers will prefer to use the notation $\mathrm{SU}(2)^{\circ 6}$.) Similarly, we will denote the embedding of six copies of the quaternion group (2.60) into $\mathrm{SU}(2)^{6}$ by $Q^{6}$ and write $Q_{6}:=Q^{6} / Z$ for the quotient by $Z$. (Again, some readers will prefer to write $Q^{\circ 6}$.)

We may consider $h(w)$ to lie in the group $Q_{6}$. There is a non-split sequence

$$
1 \rightarrow \mathbb{Z}_{2} \rightarrow Q_{6} \stackrel{\pi}{\rightarrow}\left(\mathbb{F}_{4}^{+}\right)^{6} \rightarrow 1
$$

and $h$ defines a section of $\pi$ with cocycle

$$
h\left(w_{1}\right) h\left(w_{2}\right)=\chi\left(w_{1}, w_{2}\right) h\left(w_{1}+w_{2}\right)
$$

given by the product of the cocycles (2.20).

$$
\chi\left(w_{1}, w_{2}\right)=\prod_{\alpha=1}^{6} \epsilon\left(x_{\alpha}, y_{\alpha}\right) .
$$

Note that this is to be distinguished from the cocycles generated by the group elements $\left(h\left(x_{1}\right), \ldots, h\left(x_{6}\right)\right) \in Q^{6}$. In the latter case, the cocycle is the 6 -tuple of cocycles defined in (2.20). This distinction will be of crucial importance in section 5.4.1 below.

We now come to a central claim for this paper: when restricted to the hexacode the cocycle defined by $h(w) \in Q_{6}$ is exactly equal to one. That is, $h$ in fact defines a group homomorphism:

$$
h\left(w_{1}\right) h\left(w_{2}\right)=h\left(w_{1}+w_{2}\right) \quad w_{1}, w_{2} \in \mathcal{H}_{6} .
$$

This fact is both surprising and significant. To prove it we write two hexacode words $w_{1}, w_{2}$ as:

$$
\begin{aligned}
& w_{1}=\left(x_{1}, x_{2}, x_{3}, x_{1}+x_{2}+x_{3}, x_{1} \underline{\bar{\omega}}+x_{2} \underline{\omega}+x_{3}, x_{1} \underline{\omega}+x_{2} \underline{\bar{\omega}}+x_{3}\right), \\
& w_{2}=\left(y_{1}, y_{2}, y_{3}, y_{1}+y_{2}+y_{3}, y_{1} \underline{\bar{\omega}}+y_{2} \underline{\omega}+y_{3}, y_{1} \underline{\omega}+y_{2} \underline{\bar{\omega}}+y_{3}\right) .
\end{aligned}
$$


Now we use the bimultiplicative property to expand out

$$
\epsilon\left(x_{1}+x_{2}+x_{3}, y_{1}+y_{2}+y_{3}\right)=\prod_{i, j=1}^{3} \epsilon\left(x_{i}, y_{j}\right)
$$

and similarly for the other two nontrivial factors. Now, gathering all the terms together we group together terms of the form $\epsilon\left(a x_{i}, b y_{j}\right)$ for each pair $(i, j)$. For the terms with $i=j$ we have in all

$$
\begin{aligned}
& \epsilon\left(x_{1}, y_{1}\right) \epsilon\left(x_{1}, y_{1}\right) \epsilon\left(\underline{\bar{\omega}} x_{1}, \underline{\bar{\omega}} y_{1}\right) \epsilon\left(\underline{\omega} x_{1}, \underline{\omega} y_{1}\right) \\
& \epsilon\left(x_{2}, y_{2}\right) \epsilon\left(x_{2}, y_{2}\right) \epsilon\left(\underline{\omega} x_{2}, \underline{\omega} y_{2}\right) \epsilon\left(\underline{\bar{\omega}} x_{2}, \underline{\bar{\omega}} y_{2}\right) \\
& \epsilon\left(x_{3}, y_{3}\right) \epsilon\left(x_{3}, y_{3}\right) \epsilon\left(x_{3}, y_{3}\right) \epsilon\left(\underline{\bar{\omega}} x_{3}, \underline{\bar{\omega}} y_{3}\right)
\end{aligned}
$$

and using the permutation property we see these all multiply to 1 . As an example of $i \neq j$ consider

$$
\begin{aligned}
\epsilon\left(x_{1}, y_{2}\right) \epsilon\left(\underline{\bar{\omega}} x_{1}, \underline{\omega} y_{2}\right) \epsilon\left(\underline{\omega} x_{1}, \underline{\bar{\omega}} y_{2}\right) & =\epsilon\left(x_{1}, y_{2}\right) \epsilon\left(x_{1}, \underline{\bar{\omega}} y_{2}\right) \epsilon\left(x_{1}, \underline{\omega} y_{2}\right) \\
& =\epsilon\left(x_{1},(1+\underline{\omega}+\underline{\bar{\omega}}) y_{2}\right) \\
& =\epsilon\left(x_{1}, 0 \cdot y_{2}\right) \\
& =1
\end{aligned}
$$

and it is similar for the other pairs.

It follows from (3.15) that

$$
P=\frac{1}{64} \sum_{w \in \mathcal{H}_{6}} h(w)
$$

is a projection operator. Note that for $w \neq 0$ we have $\operatorname{Tr}(h(w))=0$ and therefore

$$
\operatorname{Tr}(P)=1
$$

Therefore $P$ is a rank one projection operator. We will show in section 3.4 that for a suitably normalized spinor $s \in \operatorname{Im} P, V_{s}$ is a superconformal current.

As a check we note that indeed $\Psi$ can in fact be written as:

$$
\Psi=\frac{1}{4} \sum_{w \in \mathcal{H}} h(w)\left|0^{6}\right\rangle
$$

One way to check (3.22) is to use the homomorphism $\pi$ defined in equation (2.32) and its extension to $\left(\mathbb{F}_{4}^{+}\right)^{6}$. Recall that this homomorphism is distinguished because it is zero or one according to whether the section $h(x)$ is diagonal or off-diagonal, respectively. So it is zero or one according to whether $h(x)$ induces a bit-flip. One now checks that:

$$
h(x)\left|0^{6}\right\rangle=\chi(x)|\pi(x)\rangle \quad x \in \mathbb{F}_{4}^{6}
$$

where

$$
\chi(x)=1^{\# \underline{0}^{\prime} s}(-1)^{\# \underline{1}^{\prime} s}(\mathrm{i})^{\# \underline{\omega}^{\prime} s}(-\mathrm{i})^{\# \underline{\omega}^{\prime} s}
$$


We can now prove (3.22) by noting that the group of automorphisms of the hexacode denoted by $H_{0} \times H_{3}$ in appendix $\mathrm{A}$ induce corresponding symmetries of $\Psi$. Using these symmetries it suffices to check that we get suitable vectors from the seed codewords (A.6). Then note that, restricted to the hexacode the fiber above $0^{6}$ is order four:

$$
\pi^{-1}\left(0^{6}\right)=\{\underline{00} \underline{00} \underline{00}, \underline{00} \underline{\bar{\omega} \bar{\omega}} \underline{\bar{\omega} \bar{\omega}}, \underline{\bar{\omega} \bar{\omega}} \underline{00} \underline{\bar{\omega} \bar{\omega}}, \underline{\bar{\omega} \bar{\omega}} \underline{\bar{\omega} \bar{\omega}} \underline{00}\}
$$

So the sixtyfour hexacode words map to sixteen different terms. As far as the phases are concerned it appears one just has to check these by hand.

Finally, we note that the image $\pi\left(\mathcal{H}_{6}\right) \subset \mathbb{F}_{2}^{6}$ is exactly the code of type [6, 4] described in section 2.1 above: as promised that code determines the $N=1$ superconformal generator.

Remark. the one-dimensional code defined by $\Psi$ is closely related to the standard code of type $[[6,0,4]]$ discussed near equation (2.62). Mainiero computed its entanglement homologies and found them to be identical. Indeed, there is a local transformation relating one to the other. Mainiero found that

$$
\Psi_{[[6,0,4]]}=\left(1 \otimes U_{1} \otimes 1 \otimes U_{2} \otimes U_{2} \otimes 1\right) \Psi
$$

where

$$
U_{1}=\frac{1}{\sqrt{2}}\left(\begin{array}{cc}
1 & 1 \\
-\mathrm{i} & \mathrm{i}
\end{array}\right) \quad U_{2}=\frac{1}{\sqrt{2}}\left(\begin{array}{cc}
1 & -1 \\
\mathrm{i} & \mathrm{i}
\end{array}\right)
$$

\subsection{Why the image of $\boldsymbol{P}$ defines a superconformal current}

We now show that, for a suitably normalized state $s \in\left(\mathbb{C}^{2}\right)^{\otimes 6}$ in the image of $P$ the vertex operator $V_{s}$ is a superconformal current with $c=6$.

Given any two states $s^{1}, s^{2} \in\left(\mathbb{C}^{2}\right)^{\otimes 6}$ we have the operator product expansion

$$
V_{s^{1}}\left(z_{1}\right) V_{s^{2}}\left(z_{2}\right) \sim \frac{\bar{s}^{1} s^{2}}{z_{12}^{3}}+\kappa_{1} \sum_{A} \frac{\bar{s}^{1} \Sigma^{A} s^{2}}{z_{12}^{2}} J^{A}+\kappa_{1}^{2} \sum_{\alpha<\beta} \frac{\bar{s}^{1} \Sigma^{A B} s^{2}}{z_{12}} J^{A} J^{B}+\kappa_{2} \frac{\bar{s}^{1} s^{2}}{z_{12}} T++\cdots
$$

where $J^{A}$ correspond to the 18 generators of $\mathrm{SU}(2)^{6}, T$ is the energy-momentum tensor and all operators on the r.h.s. are evaluated at $z_{2}$. We use a composite index $A=(\alpha, i)$. Here $1 \leq i \leq 3$ label a basis of three generators of $\mathrm{SU}(2)$ and $1 \leq \alpha \leq 6$. (Later we will interpret $\alpha$ as the column number in the Miracle Octad Generator.) Then $\Sigma^{A}$ is a Pauli matrix for the column $\alpha$ :

$$
\Sigma^{A}=1 \otimes \cdots \otimes\left(\sigma^{i}\right)^{(\alpha)} \otimes \cdots 1
$$

The conjugate spinor is defined by

$$
\bar{s}:=s^{t r} \bar{\gamma}
$$

where

$$
\bar{\gamma}=\left(\mathrm{i} \sigma^{2}\right)^{(1)} \otimes \cdots \otimes\left(\mathrm{i} \sigma^{2}\right)^{(6)}
$$


is a symmetric matrix. The constant $\kappa_{1}$ depends on our normalization of $\mathrm{SU}(2)$ currents. With the convention (again dropping cocycle factors)

$$
\begin{aligned}
J^{ \pm} & \sim e^{ \pm \mathrm{i} \sqrt{2} X} \\
J^{3} & =-\mathrm{i} \partial X
\end{aligned}
$$

We find $\kappa_{1}=1 / \sqrt{2}$. Specializing to $s^{1}=s^{2}=s$ and using the symmetry properties of the Pauli matrices so that $\bar{s} \Sigma^{A} s=0$ the above equation simplifies to

$$
V_{s}\left(z_{1}\right) V_{s}\left(z_{2}\right) \sim \frac{\bar{s} s}{z_{12}^{3}}+\kappa_{1}^{2} \sum_{\alpha<\beta} \frac{\bar{s} \Sigma^{A B} s}{z_{12}} J^{A} J^{B}+\frac{1}{2} \frac{\bar{s} s}{z_{12}} T++\cdots
$$

Comparison with line one of equation (B.3) shows that the conditions for $V_{s}$ to be a superconformal current are that

$$
\bar{s} \Sigma^{A B} s=0 \quad 1 \leq \alpha<\beta \leq 6
$$

once these equations are satisfied one can normalize $s$ to achieve the desired OPE of the supercurrent. In the present case $c=6$ corresponds to $\bar{s} s=1$.

The quadratic equations in (3.34) are, as far as we are aware, all independent so there are $\left(\begin{array}{l}6 \\ 2\end{array}\right) \times 9=135$ independent equations on 64 variables. Nevertheless, if $s \in \operatorname{Im} P$ then indeed the equations are satisfied. To see this, we define a real structure on $\left(\mathbb{C}^{2}\right)^{\otimes 6}$ so that the basis $[a b c \ldots]$ are real vectors. Then

$$
\bar{\Psi}=\mathrm{i} \Psi^{\dagger}
$$

so $\bar{\Psi} \Sigma^{A B} \Psi=0$ iff $\Psi^{\dagger} \Sigma^{A B} \Psi=0$. We will now proceed to check that these equations indeed hold. Since $\Sigma^{A B}$ represent bit-flip and/or phase-flip errors on two Qbits, this is indeed the quantum error-detecting property of the code! Note that this is really a property of the quantum code, and not the classical code. For example.

$$
\begin{aligned}
\Sigma^{(1,1),(2,1)} \Psi= & {[12]+\mathrm{i}[3456]+([34]+[123456]+[56]) } \\
& +\mathrm{i}([\emptyset]+[1256]+[1234])+([235]+[145]+[136]+[246]) \\
& -\mathrm{i}([146]+[135]+[236]+[245]) \\
\Sigma^{(1,1),(2,2)} \Psi= & -[12]+\mathrm{i}[3456]+([34]-[123456]+[56]) \\
& +\mathrm{i}([\emptyset]-[1256]-[1234])+(-[235]+[145]+[136]-[246]) \\
& +\mathrm{i}(-[146]+[135]-[236]-[245])
\end{aligned}
$$

All the classical code words are the same as in $\Psi$, but, remarkably, these vectors are in fact orthogonal to $\Psi$.

The symmetry properties of the hexacode mentioned near (A.6) imply similar symmetry properties of $\Psi$ that allow us to map the general case of $\Sigma^{A B}$ to two cases: first, if $\alpha, \beta$ are in the same couple then by permuting within couples and using the cyclic symmetry 
of the 3 Pauli matrices we reduce to the two cases above. Second, if $\alpha, \beta$ are in different couples then we need to check orthogonality to

$$
\Sigma^{(1, i),(3, j)}
$$

and then we need only check $(i, j)=(1,1)$ and $(i, j)=(1,2)$. But in this case both $i, j$ involve bit-flips and one easily checks that a pair of bit-flips on 1,3 maps every term of $\Psi$ to a vector orthogonal to every term in $\Psi$. So, rather trivially, $\Sigma^{A B} \Psi$ is orthogonal to $\Psi$ for these cases. Thus $\bar{\Psi} \Sigma^{A B} \Psi=0$ for all $A B$ with $\alpha \neq \beta$. Finally, since $\bar{\Psi} \Psi=16 \mathrm{i}$, we conclude that $V_{s}$ with $s=\frac{e^{-\mathrm{i} \pi / 4}}{4} \Psi$ defines a superconformal current with $c=6$.

\subsection{The relation of the $N=4$ supercurrents to codes}

Now that we have understood the code underlying $N=1$ superconformal symmetry we can easily describe the $N=4$ supercurrents in terms of the hexacode.

For each $x \in \mathbb{F}_{4}$ let $\mathcal{H}_{6}^{x}$ denote the set of hexacode words whose first digit is $x$, and let

$$
P_{x}:=\frac{1}{16} \sum_{w \in \mathcal{H}_{6}^{x}} h(w)
$$

Note that $\mathcal{H}_{6}^{0}$ is a linear subspace of $\mathcal{H}_{6}$. In fact, it has 16 elements and is therefore a two-dimensional subspace. One choice of basis is

$$
u_{1}=(\underline{0}, \underline{0}, \underline{0}, \underline{0}, \underline{1}, \underline{1}) \quad u_{2}=(\underline{0}, \underline{\bar{\omega}}, \underline{\bar{\omega}}, \underline{0}, \underline{\omega}, \underline{1}) .
$$

The $\mathcal{H}_{6}^{x}$ are the cosets of $\mathcal{H}_{6}^{0}$ in $\mathcal{H}_{6}$ and therefore each has sixteen elements. It follows that

$$
P_{x} P_{y}=P_{x+y}
$$

Now, $P_{\underline{0}}$ is a projection operator and its image is 4-dimensional. The first $\mathrm{SU}(2)$ factor in $\mathrm{SU}(2)^{6}$ will be interpreted as the $R$-symmetry group. Note that it commutes with $P_{\underline{0}}$ and therefore $\operatorname{Im}\left(P_{\underline{0}}\right)$ is an $\mathrm{SU}(2)_{R}$ representation. Focusing on the first Qbit, $|\pi(x)\rangle$ span a two-dimensional space so the representation is $\mathbf{2} \oplus \mathbf{2}$. The four supercurrents will span the image of $P_{\underline{0}}$. In fact, we have

$$
\begin{aligned}
& \Psi^{1}=P_{\underline{0}}\left(1+P_{\underline{\omega}}\right)\left|+{ }^{6}\right\rangle \\
& \Psi^{2}=P_{\underline{0}}\left(-1+P_{\underline{\omega}}\right)\left|+{ }^{6}\right\rangle \\
& \bar{\Psi}^{1}=P_{\underline{0}}\left(P_{\underline{1}}+P_{\underline{\omega}}\right)\left|+{ }^{6}\right\rangle \\
& \bar{\Psi}^{2}=P_{\underline{0}}\left(-P_{\underline{1}}+P_{\underline{\omega}}\right)\left|+{ }^{6}\right\rangle
\end{aligned}
$$

(Note that $\Psi^{2}=\exp \left[-\mathrm{i} \pi T^{2}\right] \Psi^{1}=h(\underline{1})^{(1)} \Psi^{1}$, and that $h(\underline{1})$ anticommutes with $h(\underline{\omega})$ and $h(\underline{\bar{\omega}})$.

\section{Remarks.}

1. The image $\pi\left(\mathcal{H}_{6}^{0}\right) \subset \mathbb{F}_{2}^{6}$ is the span of the vectors $w_{1}, w_{2}, w_{3}$ in the $[6,3]$ subcode of the truncated Hamming code, as described in section 2.1.

2. For any nonzero $x \in \mathbb{F}_{4}, \mathcal{H}_{6}^{0}+\mathcal{H}_{6}^{x}$ is closed under vector addition and therefore $\frac{1}{2}\left(P_{\underline{0}}+P_{x}\right)$ is a projector to a two-dimensional subspace of $\operatorname{Im}\left(P_{\underline{0}}\right)$. This defines an embedding of an $N=2$ subalgebra in the $N=4$ superconformal algebra. 


\section{Relation to Mathieu moonshine}

\subsection{Statement of Mathieu moonshine}

The RR subspace of the space of states of a K3 sigma model is a representation of the $(4,4)$ superconformal algebra and admits an isotypical decomposition

$$
\mathcal{H}_{R R}=\oplus_{i, j} D_{i ; j} \otimes R_{i} \otimes \tilde{R}_{j}
$$

where $R_{i}$ runs over unitary irreps of leftmoving $\mathrm{N}=4$ and $\tilde{R}_{j}$ runs over unitary irreps of rightmoving $N=4$. In particular $i$ or $j$ corresponds to a pair $(h, \ell)$ with $h \geq 1 / 4, \ell=1 / 2$ for $h>1 / 4$ and $\ell \in\{0,1 / 2\}$ when $h=1 / 4$.

The remarkable Mathieu Moonshine conjecture [35] is that the virtual ${ }^{5}$ degeneracy space

$$
\mathcal{D}_{i}:=D_{i ; \tilde{h}=1 / 4, \tilde{\ell}=0}-2 D_{i ; \tilde{h}=1 / 4, \tilde{\ell}=1 / 2}
$$

for $i$ with $h>1 / 4$ is, in some natural (but thus far unexplained) way, a representation of the finite simple group $M_{24}$. Moreover, these $M_{24}$ representations have the property that the character-valued extension of the elliptic genus exhibits modular properties. This is remarkable because, thanks to the quantum Mukai theorem described below, $M_{24}$ is not the quotient of a group of $(4,4)$-preserving automorphisms of any single K3 sigma model. The significance of the virtual degeneracy space (4.2) arises from consideration of the elliptic genus. The Witten index of R-sector irreducible representations of the $\mathrm{N}=4$ superconformal algebra is:

$$
\operatorname{Tr}_{R_{(h, \ell)}} e^{2 \pi \mathrm{i} J_{0}^{3}} q^{L_{0}-c / 24}= \begin{cases}1 & h=1 / 4, \ell=0 \\ -2 & h=1 / 4, \ell=1 / 2 \\ 0 & h>1 / 4, \ell=1 / 2\end{cases}
$$

Therefore, if $g \in \operatorname{Aut}(\mathcal{C})$ is any finite-order automorphism of a $\mathrm{K} 3$ sigma model $\mathcal{C}$ that commutes with the $(4,4)$ superconformal algebra then the twisted character

$$
\begin{aligned}
\mathcal{E}_{g}(z, \tau) & :=\operatorname{Tr}_{\mathcal{H}_{R R}} g e^{2 \pi \mathrm{i}\left(J_{0}^{3}+\tilde{J}_{0}^{3}\right)} q^{L_{0}-c / 24} e^{2 \pi \mathrm{i} z J_{0}^{3}} \bar{q}^{\tilde{L}_{0}-c / 24} \\
& =\sum_{i} \operatorname{Tr}_{\mathcal{D}_{i}}(g) \chi_{i}(\tau, z)
\end{aligned}
$$

must be a weight zero index one Jacobi form for a congruence subgroup of index determined by the order of $g$. Here $\chi_{i}(\tau, z)$ are characters of irreducible representations of the (leftmoving) $N=4$ algebra:

$$
\chi_{h, \ell}(z, \tau):=\operatorname{Tr}_{R_{(h, \ell)}} e^{2 \pi \mathrm{i} J_{0}^{3}} e^{2 \pi \mathrm{i} z J_{0}^{3}} q^{L_{0}-c / 24}
$$

The outcome of further investigations $[11,36,39,45]$ is that there exist an infinite set of representations of the group $M_{24}$ :

$$
H_{0,0}, H_{0,1 / 2}, H_{n} \quad n \geq 1
$$

\footnotetext{
${ }^{5}$ On the Kummer locus the spaces $D_{i ; \tilde{h}=1 / 4, \tilde{\ell}=1 / 2}$ with $h_{i}>1 / 4$ are nonzero but there is a general expectation that these spaces vanish off the Kummer locus.
} 
(where $H_{0,0}=\mathbf{2 3}-3 \cdot \mathbf{1}$ and $H_{0,1 / 2}=-2 \cdot \mathbf{1}$, but all the other $H_{n}$ are true, not virtual, representations) such that for every $g \in M_{24}$ the function

$$
\hat{\phi}_{g}(z, \tau):=\operatorname{Tr}_{H_{0,0}}(g) \chi_{h=1 / 4, \ell=0}+\operatorname{Tr}_{H_{0,1 / 2}}(g) \chi_{h=1 / 4, \ell=1 / 2}+\sum_{n=1}^{\infty}\left(\operatorname{Tr}_{H_{n}}(g)\right) \chi_{n+1 / 4, \ell=1 / 2}
$$

transforms as if $g \in M_{24}$ acted on $\mathcal{C}$ as a $(4,4)$-preserving automorphism. Moreover

$$
\hat{\phi}_{g}(z, \tau)=\mathcal{E}_{g}(z, \tau)
$$

in those cases where $g$ is truly a $(4,4)$-preserving automorphism of the CFT. However, we stress again that there is no known natural action of $M_{24}$ on the spaces $\mathcal{D}_{i}$. We note however that in [95] A. Taormina and K. Wendland discuss how a certain maximal subgroup of $M_{24}$, the octad group, acts on a $45+45^{*}$ dimensional subspace of the states of $(h, \bar{h})=(5 / 4,1 / 4)$. Some extensions of this work appear in $[42,75]$.

The most natural way to explain the Mathieu Moonshine phenomenon would be to find some K3 sigma model with a $(4,4)$-preserving automorphism group that has a quotient that contains $M_{24} \cdot{ }^{6}$ However, the quantum Mukai theorem of Gaberdiel, Hohenegger, and Volpato, reviewed in section 4.3 below is a powerful no-go theorem that implies that such an explanation of Mathieu Moonshine cannot work. Thus, one must relax some of the hypotheses of the quantum Mukai theorem.

\subsection{The Mukai theorem}

When a K3 surface is given a complex structure it is holomorphic symplectic. The Mukai theorem characterizes the possible groups of holomorphic symplectic automorphisms of K3 surfaces. For a nice review see [80]. Once a K3 surface is endowed with a complex structure the 24-dimensional cohomology space has a Hodge decomposition

$$
H^{*}(K 3 ; \mathbb{C}) \cong H^{0,0} \oplus H^{2,0} \oplus H^{1,1} \oplus H^{0,2} \oplus H^{2,2}
$$

Any holomorphic automorphism must preserve these five components. The Mukai theorem says that all groups of holomorphic symplectic automorphisms are subgroups of $M_{23}$ with at least 5 orbits in the natural action of $M_{23}$ on a set of 24 elements. (The group $M_{24}$ has a natural action as a permutation subgroup acting on a set with 24 elements. The subgroup $M_{23}$ is isomorphic to any subgroup preserving one element.)

\subsection{Quantum Mukai theorem}

Motivated by the discovery of Mathieu Moonshine, Gaberdiel, Hohenegger, and Volpato (GHV) gave a characterization of the potential automorphism groups of supersymmetric K3 sigma models that preserve $(4,4)$ supersymmetry. The answer is, remarkably, that the groups are precisely the subgroups of the Conway group that preserve sublattices of

\footnotetext{
${ }^{6}$ We stress that all that is needed is that some quotient of the automorphism group contains $M_{24}$ as a subgroup. The action of the (4,4)-preserving automorphism group $G$ on any given degeneracy space $D_{i ; j}$ might, in general, have a kernel. Note that a different quotient, not containing $M_{24}$ as a subgroup, could act on the massless states where $h_{i}=1 / 4$, thus explaining why these are not true $M_{24}$ representations.
} 
the Leech lattice of rank greater than or equal to four [40]. In order to prove the theorem one follows $[3,4]$ to characterize a $\mathrm{K} 3$ sigma model as a choice of positive definite four-dimensional subspace of the Grassmannian $O(4,20) / O(4) \times O(20)$. A clever argument transfers the problem from a question about spaces of indefinite signature $(4,20)$ to questions about actions on the Leech lattice, a positive definite lattice of rank 24. (The proof is elegantly summarized in [66]. See also [93] for related remarks.)

Now the subgroups of the Conway group that preserve sublattices of the Leech lattice have been tabulated in [61]. None of the relevant groups contains $M_{24}$ as a subgroup of a quotient. (Also, although this is less relevant, many groups cannot be embedded as subgroups of $M_{24}$.) The Quantum Mukai Theorem is thus a powerful no-go statement in the search for an explanation of Mathieu Moonshine.

Among the rank four Höhn-Mason groups there is a distinguished maximal subgroup, $2^{8}: M_{20}$. This is closely related to the GTVW model [41]. Clearly such a special point deserves special attention. Using the relation of supercurrents to codes we will give a different derivation of the main result of [41]. Our approach makes it clear that a maximal subgroup of the Mathieu group, namely the sextet group, acts as a group of $(1,1)$-preserving automorphisms of the model.

\subsection{Evading a no-go theorem}

Given the powerful no-go theorem of GHV, any explanation of Mathieu Moonshine must proceed by relaxing one of the hypotheses in the theorem.

Two ways of relaxing the hypotheses have been explored in the past, but, at least thus far, have only met with partial success. One approach is to posit that $M_{24}$ acts not as an automorphism of the full conformal field theory but only as an "automorphism of the subspace of BPS states." The BPS states are the $N=4$ primaries with leftmoving quantum numbers $(h=1 / 4+n, \ell=1 / 2)$ and right-moving quantum numbers $(\tilde{h}=1 / 4, \ell=0,1 / 2)$. To make sense of this idea one would need some ope-like algebra structure on these BPS states. We could call this the "algebra of BPS states" approach following [56, 57]. There has been some success with this approach in the context of moonshine [89], but not yet in the context of Mathieu moonshine. A second approach is to attempt to "combine" the symmetries of different K3 sigma models at different points in the moduli space. This is the "symmetry-surfing" approach that has been vigorously pursued by A. Taormina and K. Wendland [93, 94].

The project described in this paper began with the observation that one could relax the assumption that the relevant group of automorphisms of the K3 sigma model commutes with $(4,4)$ supersymmetry. Thus, we are imagining that the $g \in M_{24}$ which can be used to define $\hat{\phi}_{g}(\tau, z)$ are in fact true automorphisms of at least some K3 sigma model but do not commute with $(4,4)$ supersymmetry. In order for the Witten index to make sense, $g$ must still commute with some right-moving $N=1$ supersymmetry. We are thus led to the idea that there might be very symmetric K3 sigma models with large symmetry groups that commute with $(4,1)$ supersymmetry, and that these symmetry groups contain $M_{24}$ as a quotient group. While this idea was an important motivation for our work, we will argue in section 5.5 below, using the twined elliptic genera, that, at least for the GTVW model, the 
enhancement from $(4,4)$ preserving to $(4,1)$ preserving symmetries will not explain $M_{24}$ symmetry. So the mystery of Mathieu Moonshine remains.

The statement of Mathieu Moonshine is only slightly altered in the $(4,1)$ case. The irreducible representations of the $N=1$ superconformal group are labeled with $(h, \epsilon)$ where $\epsilon$ is a sign given by the action of $(-1)^{F}$ on the groundstate and $h \geq c / 24$ for unitarity. Only the representations with $h=1 / 4$ have nonvanishing Witten index. Working out the branching rules for $N=4$ representations in terms of $N=1$ representations the only new point is that the virtual degeneracy spaces relevant to the elliptic genus are now

$$
\mathcal{D}_{i}^{(4,1)}:=D_{i ; h=1 / 4,+}^{(4,1)}-4 D_{i ; h=1 / 4,-}^{(4,1)}
$$

Remark: in the spirit of looking for larger automorphism groups by reducing the amount of preserved supersymmetry it is natural to ask if one could consider instead the automorphisms of K3 sigma models that commute with $(2,1)$ supersymmetry. This would indeed be possible if all the representations in (4.6) were true $M_{24}$ representations. Unfortunately, because the massless ones are virtual, and the branching of massless $\mathrm{N}=4$ reps to $\mathrm{N}=2$ reps contains infinitely many massive $\mathrm{N}=2$ reps, the virtual representations make infinitely many massive representations into virtual representations of $M_{24}$. Once one admits infinite numbers of virtual representations Moonshine becomes unsurprising.

\section{$5 \quad$ Symmetries of supercurrents}

The construction of supercurrents from quantum error correcting codes provides new insight into the subgroup of the symmetry group that stabilizes the supercurrents. We are mainly interested in the group preserving certain superconformal structures in the GTVW model but will also comment on the SCFTs with moonshine for the Conway group.

\subsection{The stabilizer group within $Q^{6}$}

As a first step to determining the symmetries of the supercurrent we note that it follows from eq. (3.15) and the expression 3.22 for $\Psi$ that $h(w) \Psi=\Psi$ for all $w \in \mathcal{H}_{6}$. Thus a copy of $\mathcal{H}_{6} \subset Q_{6}$ is a group of symmetries of the supercurrent.

Now, recalling the definition (3.11) we would like to lift this to the group $Q^{6} \subset \mathrm{SU}(2)^{6}$ of bit-flip and phase-flip errors. Recall that

$$
1 \rightarrow Z \rightarrow Q^{6} \rightarrow Q_{6} \rightarrow 1
$$

We now determine the stabilizer group $\operatorname{Stab}_{Q^{6}}(\Psi)$. We claim this is the non-abelian group

$$
\operatorname{Stab}_{Q^{6}}(\Psi)=\left\{\left(\epsilon^{1} h\left(x_{1}\right), \ldots, \epsilon^{6} h\left(x_{6}\right)\right) \mid \prod_{\alpha=1}^{6} \epsilon^{\alpha}=1 \text { and }\left(x_{1}, \ldots, x_{6}\right) \in \mathcal{H}_{6}\right\}
$$

To prove this, we note that the elements of $Q^{6}$ can be written as $\left(\epsilon^{\alpha} h\left(x_{\alpha}\right)\right)_{\alpha=1}^{6}$. Let $w=\left(x_{1}, \ldots, x_{6}\right) \in \mathbb{F}_{4}^{6}$. Then we need to solve

$$
\prod_{\alpha=1}^{6} \epsilon^{\alpha} h(w) \Psi=\Psi
$$


Now, note that $h(w)$ for $w \in \mathbb{F}_{4}^{6}$ form a linear basis for $\operatorname{End}\left(\left(\mathbb{C}^{2}\right)^{\otimes 6}\right)$. This follows since $h(x)$ for $x \in \mathbb{F}_{4}$ form a linear basis for the complex vector space $\operatorname{End}\left(\mathbb{C}^{2}\right)$. Therefore $h(w) \Psi$ for $w \in \mathcal{H}_{6}^{\text {comp }}$ form a linear basis for $\left(\mathbb{C}^{2}\right)^{\otimes 6}$. This follows because the vectors $h(w) \Psi$ for $w \in \mathbb{F}_{4}^{6}$ generates the entire vector space. But every $w \in \mathbb{F}_{4}^{6}$ can be written as $w=u_{1}+u_{2}$ with $u_{1} \in \mathcal{H}_{6}^{\text {comp }}$ and $u_{2} \in \mathcal{H}_{6}$ (as we saw in equation (2.35) et. seq. above) and then $h(w) \Psi= \pm h\left(u_{1}\right) \Psi$. So these must generate the entire vector space. But there are at most $4^{3}=2^{6}$ linearly independent vectors $h\left(u_{1}\right) \Psi$, so these must in fact be linearly independent. It follows that if there is a $w \in \mathbb{F}_{4}^{6}$ such that $h(w) \Psi= \pm \Psi$ then the sign must be + and $w \in \mathcal{H}_{6}$.

Thus we have

$$
1 \rightarrow Z \rightarrow \operatorname{Stab}_{Q^{6}}(\Psi) \rightarrow \mathcal{H}_{6} \rightarrow 0
$$

where the reader will recall that $Z$ is the subgroup of the center of $\mathrm{SU}(2)^{6}$ that acts ineffectively on the 6 Qbit system.

\subsection{Further symmetries of $\Psi$ : lifting the hexacode automorphisms}

The group $H:=\mathrm{SU}(2)^{6}: S_{6}$ acts on the six Qbit system in a natural way, and this group can be lifted to a symmetry group of the chiral part of the GTVW model. In sections 5.4.1 and 5.4.2 below we discuss the lift to the full GTVW model. As a preliminary, it is therefore useful to discuss what we know about $\operatorname{Stab}_{H}(\Psi)$. Here it is very useful to observe that automorphisms of the hexacode lift to operators on the Q-bit system that commute with the projector $P$ defined in (3.20).

To demonstrate this we use the description of the automorphism group of the hexacode in appendix A. The generators $g_{1}, \ldots, g_{4}$ are pure permutations. Letting $\hat{g}_{1}, \ldots, \hat{g}_{4}$ denote the corresponding permutations acting on the factors of the six Qbit Hilbert space we clearly have

$$
\hat{g}_{i} h(w) \hat{g}_{i}^{-1}=h\left(g_{i} \cdot w\right)
$$

for all $w \in \mathbb{F}_{4}^{6}$ and all $i=1,2,3,4$ and therefore $\hat{g}_{i}$ commutes with $P$. For $g_{0}$ we note that $h(\omega x)=\Omega^{-1} h(x) \Omega$ and therefore letting $\hat{g}_{0}:=\left(\Omega^{-1}\right)^{\otimes 6}$ we have

$$
\hat{g}_{0} h(w) \hat{g}_{0}^{-1}=h\left(g_{0} \cdot w\right)
$$

for all $w \in \mathbb{F}_{4}^{6}$ and therefore $\hat{g}_{0}$ commutes with $P$. Similarly, define

$$
\hat{g}_{5}:=(465) \cdot\left(\Omega^{-1} \otimes \Omega \otimes 1 \otimes 1 \otimes 1 \otimes 1\right)
$$

so that

$$
\hat{g}_{5} h(w) \hat{g}_{5}^{-1}=h\left(g_{5} \cdot w\right)
$$

for all $w \in \mathbb{F}_{4}^{6}$. Accordingly, $\hat{g}_{5}$ commutes with $P$.

Finally, we define a lift of $g_{F}$. It is easy to prove that there is no linear operator that implements the Frobenius automorphism on $\mathbb{F}_{4}$. That is, there is no matrix $A$ such that $A h(x) A^{-1}=h(\bar{x})$. Nevertheless, if we define

$$
v:=\frac{\mathrm{i}}{\sqrt{2}}\left(\sigma^{1}+\sigma^{3}\right)=\frac{\mathrm{i}}{\sqrt{2}}\left(\begin{array}{cc}
1 & 1 \\
1 & -1
\end{array}\right)
$$


one can check that $v^{2}=-1$ and

$$
v h(x) v^{-1}=h(x)^{\dagger}= \begin{cases}h(x) & x=\underline{0} \\ -h(\bar{x}) & x \neq \underline{0}\end{cases}
$$

Define $\hat{g}_{F}=(56) \cdot v^{\otimes 6}$. Then, since there are always an even number of nonzero digits in a hexacode word we do in fact have

$$
\hat{g}_{F} h(w) \hat{g}_{F}^{-1}=h\left(g_{F} \cdot w\right)
$$

when $w \in \mathcal{H}_{6}$, and this is sufficient to prove that $\hat{g}_{F}$ commutes with $P$.

Thus, the lifted elements $\hat{g}_{0}, \ldots, \hat{g}_{5}, \hat{g}_{F}$ generate a group that commutes with $P$. Therefore, since the image of $P$ is one-dimensional we can say that

$$
\hat{g}_{i} \cdot \Psi=\xi_{i} \Psi \quad i=1, \ldots, 7
$$

for some phase $\xi_{i}$. Now, for $\hat{g}_{1}, \ldots, \hat{g}_{4}$, a simple direct check shows that $\xi_{i}=+1$ for $i=1,2,3,4$. We must work a bit harder to find $\xi_{0}, \xi_{5}$, and $\xi_{F}$.

Since $\hat{g}_{0}^{3}=1$ it follows that $\xi_{0}$ is a third root of unity. We claim that, in fact, $\xi_{0}=1$. To prove this we use the reality properties of $\Psi$. Define the symmetric matrix:

$$
\bar{\gamma}=\left(\mathrm{i} \sigma^{2}\right)^{(1)} \otimes \cdots \otimes\left(\mathrm{i} \sigma^{2}\right)^{(6)}
$$

and compute:

$$
\bar{\gamma} \Psi=\mathrm{i} \Psi^{*} .
$$

Now recall that $\Omega^{*}=-\left(\mathrm{i} \sigma^{2}\right) \Omega\left(\mathrm{i} \sigma^{2}\right)$ which implies that $\hat{g}_{0}^{*}=\bar{\gamma} \hat{g}_{0} \bar{\gamma}$ and then (5.14) implies that $\xi_{0}^{*}=\xi_{0}$ and therefore $\xi_{0}=1$. The same style of argument shows that $\xi_{5}=+1$.

It is worth noting that since $\hat{g}_{i} \Psi=\Psi$ for $i=0, \ldots, 5$ it follows that the group $\left\langle\hat{g}_{0}, \ldots, \hat{g}_{5}\right\rangle$, which is, a priori, only an extension of $H_{05}:=\left\langle g_{0}, \ldots, g_{5}\right\rangle$ is in fact isomorphic to $H_{05}$.

Finally, we note that $\hat{g}_{F}$ has order two. A direct computation of $\left\langle+^{6}\left|\hat{g}_{F}\right| \Psi\right\rangle$ shows that in fact

$$
\hat{g}_{F} \Psi=-\Psi
$$

so $\hat{g}_{F}$ is not in the stabilizer group. However we can remedy this by defining $\check{g}_{F}$ to be the product of $\hat{g}_{F}$ with the transformation $\left(-1,1^{5}\right) \in \mathrm{SU}(2)^{6}$ (or any other element of the center of $\mathrm{SU}(2)^{6}$ that is not in $\left.Z\right) .{ }^{7}$ Then

$$
\check{g}_{F} \Psi=\Psi
$$

As discussed in appendix A the elements $g_{0}, \ldots, g_{5}, g_{F}$ generate $\operatorname{Aut}\left(\mathcal{H}_{6}\right)$ isomorphic to $\mathbb{Z}_{3} . S_{6}$. The lifts $\hat{g}_{0}, \ldots, \hat{g}_{5}, \check{g}_{F}$ stabilize $\Psi$ and generate a group isomorphic to $\mathbb{Z}_{3} . S_{6}$. Of course, the "translation" action by hexacode elements themselves stabilize $\Psi$ and so the semidirect product

$$
2^{6}: 3 . S_{6} \subset \mathrm{SU}(2)_{6}: S_{6}
$$

stabilizes $\Psi$.

\footnotetext{
${ }^{7}$ We thank T. Johnson-Freyd for pointing this out to us.
} 


\section{Remarks:}

1. What we have described above as the stabilizer of $\Psi$ is the holomorph of the hexacode, $\operatorname{Hol}\left(\mathcal{H}_{6}\right)$. See appendix A for a definition of the term "holomorph."

2. We still must lift the above symmetry group in $\mathrm{SU}(2)_{6}: S_{6}$ to a symmetry of the full GTVW Hilbert space. There are two issues involved when doing this. First, lifting from a subgroup of $\mathrm{SU}(2)_{6}$ to $\mathrm{SU}(2)^{6}$ involves an extension by the subgroup $Z$ of the center of $\mathrm{SU}(2)^{6}$. Second, including left-movers with right-movers, those automorphisms that involve a nontrivial permutation of hexacode digits must be embedded diagonally in the product of left- and right-moving hexacode holomorphs. These aspects will be carefully described in sections 5.4 .1 and 5.4.2 below.

\subsection{The stabilizer group of $\operatorname{Im}(P)$ within $\mathrm{SU}(2)^{6}$}

The question now arises as to the nature of the full stabilizer group within the automorphism group $\mathrm{SU}(2)^{6}: S_{6}$ of the chiral algebra $\mathcal{V}_{\overrightarrow{0}}$ of the GTVW model.

One nice consequence of the error-detecting code description of $\Psi$ is that the stabilizer within $\mathrm{SU}(2)^{6}$ is a discrete group. To show this we consider the $h\left(x_{i}\right)$ for $x_{i} \in \mathbb{F}_{4}^{*}$ to be generators of the Lie algebra $\mathfrak{s u}(2)$. Note well that in this sub-section we are not thinking of these matrices multiplicatively!

We can show that the stabilizer group of $\Psi$ is discrete by showing that there are no nontrivial solutions of

$$
\sum_{x \in \mathbb{F}_{4}^{*}} \sum_{\alpha=1}^{6} c_{x, \alpha} h(x)_{\alpha} \Psi=0
$$

where $h(x)_{\alpha}$ means that the matrix only acts on the $\alpha^{\text {th }}$ factor. The computation, which is slightly technical, is relegated to appendix C. The main point though, is that it is a again true due to the error correcting properties of $\Psi$.

In fact, the stabilizer is a finite group. If the stabilizer were discrete and infinite, then, being a subgroup of the compact group $\mathrm{SU}(2)^{6}$ there would be an accumulation point. We can rule out this possibility by noting that in fact the stabilizer group is an algebraic group. Indeed, it can be characterized as the solutions to

$$
\langle\Psi|u| \Psi\rangle=\langle\Psi \mid \Psi\rangle
$$

which constitutes a (complicated) algebraic equation for the matrix elements of $u \in \mathrm{SU}(2)^{6}: S_{6}$.

In [67] T. Johnson-Freyd has discussed the automorphism groups of holomorphic $N=1$ superVOA's in a large class of models. It turns out that the GTVW model is a special case of the class of models considered in [67]. Using the methods of [67], and the relation of the GTVW model to a theory of $12 \mathrm{MW}$ fermions, one can show that the symmetry group of $\Psi$ can in fact be no larger than the holomorph of the hexacode. Given our result above, it is exactly the holomorph of the hexacode. 


\subsection{Extending the automorphism groups to include left- and right-movers}

The $\mathrm{SU}(2) k=1$ WZW model has, famously, $\widehat{\mathrm{SU}(2)}_{L}^{k=1} \times \widehat{\mathrm{SU}(2)}_{R}^{k=1}$ current algebra symmetry and the global $\mathrm{SU}(2)_{L} \times \mathrm{SU}(2)_{R}$ symmetry is an automorphism of the model. (An automorphism of the sigma model should certainly preserve the conformal weights, and hence we only take the subgroup of the affine Lie group that commutes with $L_{0}$ and $\tilde{L}_{0}$.) Actually, the diagonally embedded center of $\mathrm{SU}(2)_{L} \times \mathrm{SU}(2)_{R}$ acts trivially so only the quotient, denoted $\mathrm{SO}(4)_{L R}$, acts effectively. On the other hand, there is a left-right reflection action of the model. It exchanges the left- and right-movers and generates an $O(4)$ action on the space of states. Elements in the nontrivial component permute conformal weights $(h, \tilde{h}) \rightarrow(\tilde{h}, h)$ and hence $O(4)$ is not an automorphism of the entire sigma model. Nevertheless, it is a global symmetry of the space of ground states and it can be useful.

When we turn to the product of six WZW models we clearly have a symmetry group $\mathrm{SO}(4)_{L R}^{6}: S_{6}$, where the permutation group $S_{6}$ permutes the 6 factors. In the GTVW spectrum (3.2) there are spinor representations so the symmetry group is in fact a quotient of $\operatorname{Spin}(4)^{6}: S_{6}$. Let $Z_{\text {diag }}$ denote the diagonal embedding of $Z \subset \mathrm{SU}(2)^{6}$ into $\left(\mathrm{SU}(2)_{L}^{6} \times \mathrm{SU}(2)_{R}^{6}\right)$. The group of symmetries acting effectively on the GTVW spectrum is $\left(\operatorname{Spin}(4)^{6} / Z_{\text {diag }}\right): S_{6}$.

Just the way there is a further parity symmetry when one considers ground states of the WZW model for a single factor SU(2), there is an $O(4)^{6}: S_{6}$ symmetry group of the set of RR ground states of the model. In the GTVW model we find spinor representations and there is a group action of $\operatorname{Pin}(4)^{6}: S_{6}$ where $\operatorname{Pin}(4)$ is the double cover of $O(4)$ that acts on spinors. ${ }^{8}$ Note that $\operatorname{Pin}(4)^{6}$ has a projection to $O(4)^{6}$ and taking the determinant of each factor gives a map to $\mathbb{Z}_{2}^{6}$. When the image is not one, some left- and right-conformal weights on some factors will be exchanged. In particular this group does not, in general preserve the space of potential supercurrents:

$$
\mathcal{V}_{1^{6}} \otimes \tilde{\mathcal{V}}_{0^{6}} \oplus \mathcal{V}_{0^{6}} \otimes \tilde{\mathcal{V}}_{1^{6}}
$$

The subgroup that preserves $\mathcal{H}_{\mathrm{GTVW}}^{3 / 2,0} \oplus \mathcal{H}_{\mathrm{GTVW}}^{(0,3 / 2)}$ is that where image of the determinant map is either all +1 or all -1 . We will denote this group as:

$$
\mathrm{P}(\operatorname{in}(4))^{6}
$$

Put simply: the spinor lift of a parity transformation is diagonally embedded in all six factors. Thus the group $\mathrm{P}(\operatorname{in}(4))^{6}: S_{6}$ acts both on the space $\mathcal{H}_{\mathrm{GTVW}}^{3 / 2,0} \oplus \mathcal{H}_{\mathrm{GTVW}}^{(0,3 / 2)}$ as well as on the RR ground states.

\subsubsection{Stabilizer of $(4,4)$ supersymmetry within $\operatorname{Spin}(4)^{6}: S_{6} / Z_{\text {diag }}$}

We are now in a good position to determine the group of symmetries of the GTVW model that commute with $(4,4)$ supersymmetry. One way to construct such symmetries proceeds

\footnotetext{
${ }^{8}$ Thanks to Bott periodicity $\operatorname{Pin}^{+}(4) \cong \operatorname{Pin}^{-}(4)$. In fact, the groups are canonically isomorphic: if $e_{i}$ is a set of Clifford generators for $\operatorname{Pin}^{ \pm}(4)$ then $\frac{1}{3 !} \epsilon_{i j k l} e_{j} e_{k} e_{l}$ is a set of Clifford generators for $\operatorname{Pin}^{\mp}(4)$. Of course, they are not isomorphic as double covers of $O(4)$.
} 
by lifting suitable subgroups of the holomorph of the hexacode to $\left(\operatorname{Spin}(4)^{6} / Z_{\text {diag }}\right): S_{6}$. We will construct a group of symmetries that is related to $M_{20}$ in a way explained in detail below. Since the discussion has several subtleties we will be going into excruciating and explicit detail.

To begin, we work chirally, and consider lifts of holomorphs of the hexacode to the semidirect product $\mathrm{SU}(2)^{6}: S_{6}$ which preserve all four chiral supersymmetries. Now, elements of $\mathrm{SU}(2)^{6}: S_{6}$ that commute with all four chiral supersymmetries must commute with the $\mathrm{SU}(2)$ R-symmetry. In the GTVW model the chiral R-symmetry is identified with the first $\mathrm{SU}(2)$ factor in the product $\mathrm{SU}(2)^{6}$. Symmetries of this type that are lifts of holomorphs of the hexacode must be lifts of holomorphs that preserve the first digit of the hexacode. Therefore we begin by determining the subgroup, $F \subset \operatorname{Aut}\left(\mathcal{H}_{6}\right)$, that fixes the first digit of the hexacode. It is easy to see that $F \subset H_{5}=\left\langle g_{1}, \ldots, g_{5}\right\rangle$. The elements $g_{2}=(34)(56)$ and $g_{4}=(35)(46)$ of appendix A are certainly in $F$ As an example of a nontrivial element of $F$ we can modify the generator $g_{5}$ in appendix A by combining with elements of $H_{0} \times H_{3}$ to get, for example:

$$
g_{5}^{\prime}:\left(x_{1}, \ldots, x_{6}\right) \mapsto\left(x_{1}, x_{5}, \underline{\omega} x_{4}, \underline{\underline{\omega}} x_{3}, x_{2}, x_{6}\right)
$$

Now recall (equation (A.10)) that there is a homomorphism $p: \operatorname{Aut}\left(\mathcal{H}_{6}\right) \rightarrow S_{6}$ that simply tracks what permutation of digits the automorphism implements. We have $p\left(g_{5}^{\prime}\right)=(25)(34)$. Now (34)(56) and (35)(46) generate a $\mathbb{Z}_{2} \times \mathbb{Z}_{2}$ subgroup of order 4 . But $(34)(56)(25)(34)=(256)$ has order 3 while $(35)(46)(25)(34)=(23645)$ has order 5 . It follows that $4 \cdot 3 \cdot 5=60$ must divide the order of $p(F)$. On the other hand, $p(F)$ is a proper subgroup of $S_{5}$ and hence $p(F)$ must be exactly $A_{5} \subset S_{5}$. The kernel of $p$ restricted to $F$ is trivial so $F$ is isomorphic to $A_{5}$.

Now, still working chirally, let us consider the "translation symmetries," that is, the action by $\mathrm{SU}(2)_{6}$ elements $h(w)$ (acting either on the left- or the right- movers) that commute with $N=4$ supersymmetry. Thanks to the description of the $N=4$ currents in section 3.5 we see that these translations by $w \in \mathcal{H}_{6}$ must commute with $P_{x}$ and hence must preserve the first digit of hexacode words. Therefore when acting with $h(w)$ the first digit of $w$ must be $\underline{0}$. Let $\mathcal{H}_{6}^{0} \subset \mathcal{H}_{6}$ be the subspace consisting of hexacode words whose first digit is $\underline{0}$. As we have seen, this is a 2-dimensional subspace over $\mathbb{F}_{4}$. It has 16 elements and, as an Abelian group, $\mathcal{H}_{6}^{0} \cong \mathbb{Z}_{2}^{4}$. Since we can independently lift a translation by a hexacode word to elements of both $\mathrm{SU}(2)_{L}^{6}$ and $\mathrm{SU}(2)_{R}^{6}$ we are led to consider the group

$$
\left(\mathcal{H}_{6, L}^{0} \times \mathcal{H}_{6, R}^{0}\right): F
$$

with $F$ acting diagonally as a group of automorphisms.

Now we must lift these group operations to $\left(\operatorname{Spin}(4)^{6} / Z_{\text {diag }}\right): S_{6}$. First, as we have seen, the lift $\left\langle\widehat{g}_{1}, \ldots, \widehat{g}_{5}^{\prime}\right\rangle$ does not generate a central extension in $\mathrm{SU}(2)_{6}: S_{6}$ and we will demonstrate below that the further lift to $\mathrm{SU}(2)^{6}: S_{6}$ is isomorphic to $A_{5}$.

At this point, the reader should recall the important remark concerning the relation of $\mathrm{SU}(2)^{6}$ to $\mathrm{SU}(2)_{6}$ mentioned in section 3.3.

In order to lift to $\left(\operatorname{Spin}(4)^{6} / Z_{\text {diag }}\right): S_{6}$ note that if $p(\varphi)$ is a nontrivial element of $S_{6}$ then it must act diagonally on the left- and right-movers. If $\varphi \in F$ we will denote its lift 
to the full GTVW model by $\widehat{\varphi}$. As discussed near (5.4), when lifting $\mathcal{H}_{6}$ to a subgroup of $\mathrm{SU}(2)^{6}$ via

$$
\left(x_{1}, \ldots, x_{6}\right) \rightarrow\left(h\left(x_{1}\right), \ldots, h\left(x_{6}\right)\right) \in \mathrm{SU}(2)^{6}
$$

we encounter an extension by $Z \cong \mathbb{Z}_{2}^{5}$. Again, we stress that the cocycle defined by this section is a 6-tuple of the cocycles (2.20), and not their product! Altogether then, we have a group $\mathcal{G}_{4,4}$ which fits in an extension:

$$
1 \rightarrow Z \rightarrow \mathcal{G}_{4,4} \stackrel{\pi}{\longrightarrow}\left(\mathcal{H}_{6, L}^{0} \times \mathcal{H}_{6, R}^{0}\right): F \rightarrow 1
$$

where $Z$ should really be regarded as $\left(Z_{L} \times Z_{R}\right) / Z_{\text {diag. }}$. More abstractly, $\mathcal{G}_{4,4}$ has the structure of a nontrivial central extension:

$$
\mathcal{G}_{4,4} \cong \mathbb{Z}_{2}^{5} \cdot\left(\left(\mathbb{Z}_{2}^{4} \times \mathbb{Z}_{2}^{4}\right): A_{5}\right)
$$

Note that

$$
\left|\mathcal{G}_{4,4}\right|=2^{15} \cdot 3 \cdot 5
$$

We will now argue that the central extension (5.25) is nontrivial.

It is possible to give a very concrete description of the group $\mathcal{G}_{4,4}$. We will write group elements in $\left.\left(\mathrm{SU}(2)_{L}^{6} \times \mathrm{SU}(2)_{R}^{6}\right)\right): S_{6}$ using the notation

$$
\left(\left(u_{1}, \ldots, u_{6}\right)_{L},\left(\tilde{u}_{1}, \ldots, \tilde{u}_{6}\right)_{R} ; \sigma\right)
$$

with $u_{i}, \tilde{u}_{i} \in \mathrm{SU}(2)$ and $\sigma \in S_{6}$. The multiplication rule is the usual semidirect product rule. As mentioned above, to obtain a group acting effectively on the CFT we must take a quotient by $Z_{\text {diag. }}$. In other words it is understood that we identify:

$$
\left(\left(u_{1}, \ldots, u_{6}\right)_{L},\left(\tilde{u}_{1}, \ldots, \tilde{u}_{6}\right)_{R} ; \sigma\right) \sim\left(\left(z_{1} u_{1}, \ldots, z_{6} u_{6}\right)_{L},\left(z_{1} \tilde{u}_{1}, \ldots, z_{6} \tilde{u}_{6}\right)_{R} ; \sigma\right)
$$

where $\left(z_{1}, \ldots, z_{6}\right) \in Z \subset Z\left(\mathrm{SU}(2)^{6}\right)$.

Now we choose a section of $\pi$ over $F$ in equation (5.25) generated by

$$
\begin{aligned}
& \widehat{g}_{2}=\left(1_{L}^{6}, 1_{R}^{6} ;(34)(56)\right) \\
& \widehat{g}_{4}=\left(1_{L}^{6}, 1_{R}^{6} ;(35)(46)\right) \\
& \widehat{g}_{5}^{\prime}=\left(\left(\left(1,1, \Omega^{-1}, \Omega, 1,1\right)_{L},\left(1,1, \Omega^{-1}, \Omega, 1,1\right)_{R}\right) ;(25)(34)\right)
\end{aligned}
$$

This section splits the sequence over $F$ and defines a subgroup of $\mathcal{G}_{4,4}$ isomorphic to $A_{5}$. It is an amusing exercise to verify that $\widehat{g}_{5}^{\prime}$ has order two, that $\widehat{g}_{2} \widehat{g}_{5}^{\prime}$ has order 3 , and that $\widehat{g}_{4} \widehat{g}_{5}^{\prime}$ has order 5 .

Now, over $\mathcal{H}_{6, L} \times \mathcal{H}_{6, R}$ we choose the section

$$
\left(\left(h\left(x_{1}\right), \ldots, h\left(x_{6}\right)\right)_{L},\left(h\left(\tilde{x}_{1}\right), \ldots, h\left(\tilde{x}_{6}\right)\right)_{R} ; 1\right)
$$

where $\left(x_{1}, \ldots, x_{6}\right) \in \mathcal{H}_{6}$ and $\left(\tilde{x}_{1}, \ldots, \tilde{x}_{6}\right) \in \mathcal{H}_{6}$. The restriction to $\mathcal{H}_{6, L}^{0} \times \mathcal{H}_{6, R}^{0}$ imposes $x_{1}=\tilde{x}_{1}=\underline{0}$ and consequently $h\left(x_{1}\right)=h\left(\tilde{x}_{1}\right)=1$. The multiplication of these group elements will clearly involve a 6 -tuple of cocycles $\epsilon(x, y)$ in equation $(2.20)$ and hence the extension by $Z$ might be nontrivial. 
There are two ways to see that the extension (5.25) is indeed nontrivial. First, note that every element of $\mathcal{H}_{6, L}^{0} \times \mathcal{H}_{6, R}^{0}$ is an involution. Consider the square of the lift of any nonidentity element in $\mathcal{H}_{6, L}^{0} \times \mathcal{H}_{6, R}^{0}$. The square will be a nonidentity element in the subgroup $Z^{0} \subset Z$ defined by the condition $z_{1}=1$. In fact, all elements of $Z^{0}$ can be obtained in this way. (Note that $Z^{0}$ is isomorphic to $\mathbb{Z}_{2}^{4}$.) On the other hand, every element in $Z$ is order two so it is impossible to produce a nonidentity element which is a perfect square. Therefore there exist involutions whose lifts have squares which are not themselves perfect squares in $Z$. This implies the extension is nontrivial. ${ }^{9}$ In fact, every nonidentity element in $\mathcal{H}_{6, L}^{0} \times \mathcal{H}_{6, R}^{0}$ provides an example.

A second way to see that the extension (5.25) is nontrivial is to note that since $\mathcal{H}_{6, L}^{0} \times \mathcal{H}_{6, R}^{0}$ is Abelian it suffices to check if the commutator of lifted group elements is trivial or not. The story is the same for left- and right-movers so we might as well take $\left(h\left(\tilde{x}_{1}\right), \ldots, h\left(\tilde{x}_{6}\right)\right)_{R}=1_{R}^{6}$. Then the commutator of the lifts of elements in $\mathcal{H}_{6, L}^{0}$ is of the form:

$$
\left(\left(c\left(x_{1}, y_{1}\right), \ldots, c\left(x_{6}, y_{6}\right)\right)_{L} \times 1_{R}^{6} ; 1\right)
$$

where $\left(x_{1}, \ldots, x_{6}\right) \in \mathcal{H}_{6}^{0}$ and $\left(y_{1}, \ldots, y_{6}\right) \in \mathcal{H}_{6}^{0}$ and the commutator function is

$$
c(x, y)=\left\{\begin{array}{lll}
1 & x=y & \text { or } \quad x y=\underline{0} \\
-1 & x \neq y & \text { and } \quad x y \neq \underline{0}
\end{array}\right.
$$

Again, a simple perusal of equation (A.14) shows that every element of $Z^{0}$ appears as a group commutator.

We can now describe the relation to the group $2^{8}: M_{20}$ obtained in [41]. Although the extension (5.25) is a nontrivial central extension it is in fact isomorphic to $2^{9}: M_{20}$ where the $2^{9}$ is a noncentral subgroup. ${ }^{10}$ For each $v \in \mathbb{F}_{4}^{*}$ we will define subgroups $\mathcal{H}_{L R}(v)$ of $\left(Q^{6} \times Q^{6}\right) / Z_{\text {diag }}$. Each subgroup $\mathcal{H}_{L R}(v)$ is isomorphic to $\mathcal{H}_{6}$. The group $\mathcal{H}_{L R}(v)$ is the group of elements

$$
\left[\left(h\left(x_{1}, \ldots, h\left(x_{6}\right)\right)_{L},\left(h\left(v x_{1}\right), \ldots, h\left(v x_{6}\right)\right)_{R}\right]\right.
$$

where the square brackets denote the equivalence class under the quotient by $Z_{\text {diag. }}$. Note that the group elements $\left(h\left(x_{1}\right), \ldots, h\left(x_{6}\right)\right) \in Q^{6}$ do not form a subgroup, even when we restrict to $\left(x_{1}, \ldots, x_{6}\right) \in \mathcal{H}_{6}$. However the group elements (5.34) do form a subgroup thanks to the division by $Z_{\text {diag }}$ and the permutation invariance of the cocycle (see equation (2.23)). Again, using permutation invariance of the cocycle, the elements of the form (5.34) are all involutions. Indeed, $\mathcal{H}_{L R}(v) \cong \mathbb{Z}_{2}^{6}$ as an Abelian group. Similarly, the restriction to the subgroup with $x_{1}=0$ defines subgroups $\mathcal{H}_{L R}^{0}(v)$ of $\mathcal{G}_{4,4}$, each of which is isomorphic to $\mathbb{Z}_{2}^{4}$. Now it is easy to check that $\mathcal{H}_{L R}(v)$ acts, via conjugation, on $\mathcal{H}_{L R}\left(v^{\prime}\right)$ for $v \neq v^{\prime}$ as a nontrivial automorphism, and similarly for $\mathcal{H}_{L R}^{0}(v)$ and $\mathcal{H}_{L R}^{0}\left(v^{\prime}\right)$ for $v \neq v^{\prime}$. Now denote by $\mathcal{G}_{4,4}^{1} \subset \mathcal{G}_{4,4}$ the subgroup defined by the fiber of $\pi$ over the "part with $F=1$ ". ${ }^{11}$ The

\footnotetext{
${ }^{9}$ For an explanation of this result the reader might wish to consult Remark 5 in section 14.3 of [84].

${ }^{10}$ The relevant isomorphism was discovered by T. Johnson-Freyd, and we thank him for extensive discussions and clarifications related to this.

${ }^{11}$ To be more precise: let $\pi^{(2)}:=p^{(2)} \circ \pi$ where $p^{(2)}:\left(\mathcal{H}_{6, L}^{0} \times \mathcal{H}_{6, R}^{0}\right): F \rightarrow F$ is the projection and define $\mathcal{G}_{4,4}^{1}$ to be the kernel of $\pi^{(2)}$.
} 
center of $\mathcal{G}_{4,4}^{1}$ is $Z$. Each of the subgroups $Z \times \mathcal{H}_{L R}^{0}\left(v^{\prime}\right) \subset \mathcal{G}_{4,4}^{1}$ is a normal subgroup. A complementary group can be taken to be $\mathcal{H}_{L R}(v)$ with $v^{\prime} \neq v$, that is we can write

$$
\mathcal{G}_{4,4}^{1} \cong\left(Z \times \mathcal{H}_{L R}^{0}\left(v^{\prime}\right)\right): \mathcal{H}_{L R}(v)
$$

for any pair $v \neq v^{\prime}$, where in the semidirect product $\mathcal{H}_{L R}(v)$ acts nontrivially on $\mathcal{H}_{L R}\left(v^{\prime}\right)$ by conjugation. ${ }^{12}$

Now, since the fibration trivializes over $F$ we can restore $F$ to write

$$
\mathcal{G}_{4,4} \cong\left(Z \times \mathcal{H}_{L R}^{0}\left(v^{\prime}\right)\right):\left(\mathcal{H}_{L R}(v): F\right)
$$

We can now make contact with the group $M_{20}$. It is known ${ }^{13}$ that

$$
M_{20} \cong \mathbb{Z}_{2}^{4}: A_{5}
$$

and so we can identify

$$
M_{20} \cong \mathcal{H}_{6}^{0}(v): F
$$

for any $v \in \mathbb{F}_{4}^{*}$. On the other hand,

$$
Z \times \mathcal{H}_{6}^{0}\left(v^{\prime}\right) \cong \mathbb{Z}_{2}^{9}
$$

and hence we obtain

$$
\mathcal{G}_{4,4} \cong \mathbb{Z}_{2}^{9}: M_{20}
$$

where only $\mathbb{Z}_{2}^{5} \subset \mathbb{Z}_{2}^{9}$ is central.

Finally, we comment on the difference of $2^{9}: M_{20}$ vs. the group $2^{8}: M_{20}$ that appears in [41]. The space of RR groundstates decomposes, under the $\mathrm{SU}(2)_{L} \times \mathrm{SU}(2)_{R} R$-symmetry group as $(\mathbf{2} ; \mathbf{2}) \oplus 20(\mathbf{1} ; \mathbf{1})$ and the theorem of $[40]$ only addresses the commutant of $(4,4)$ supersymmetry that acts trivially on the subspace $(\mathbf{2} ; \mathbf{2})$ of RR states. Comparing with equation (6.1) for the RR groundstates in the GTVW model we see that to compare results we should only consider the subgroup of $\mathcal{G}_{4,4}$ that acts trivially on the space $(\mathbf{2} ; \mathbf{2})_{\alpha=1}$. This subgroup, $\mathcal{G}_{4,4}^{0}$ is obtained by restriction of the semidirect product (5.36) obtained by replacing $Z$ with the subgroup $Z^{0} \subset Z$ with $z_{1}=1$. That subgroup is isomorphic to $\mathbb{Z}_{2}^{4}$. The "extra" $\mathbb{Z}_{2}$ can be generated by $\left(\left(-1_{L}^{6}, 1_{R}^{6}\right) ; 1\right)$ and this element can be interpreted as $(-1)^{F_{R}}$. Thus,

$$
\mathcal{G}_{4,4}^{0} \cong 2^{8}: M_{20}
$$

in accord with [41]. Note again that the $2^{8}$ is noncentral.

\footnotetext{
${ }^{12}$ To make this completely explicit, the main point is to note that for all $a$ and $b$ we can solve $\left[\left(h\left(a_{1}\right), \ldots, h\left(a_{6}\right)\right)_{L},\left(h\left(b_{1}\right), \ldots, h\left(b_{6}\right)\right)_{R}\right]$ $=z \cdot\left[\left(h\left(x_{1}\right), \ldots, h\left(x_{6}\right)\right)_{L},\left(h\left(v x_{1}\right), \ldots, h\left(v x_{6}\right)\right)_{R}\right] \cdot\left[\left(h\left(y_{1}\right), \ldots, h\left(y_{6}\right)\right)_{L},\left(h\left(v^{\prime} y_{1}\right), \ldots, h\left(v^{\prime} y_{6}\right)\right)_{R}\right]$

for some $z \in Z$ and $x, y \in \mathcal{H}_{6}$. To see this note that for each $\alpha$ we need to solve $x_{\alpha}+y_{\alpha}=a_{\alpha}$ and $v x_{\alpha}+v^{\prime} y_{\alpha}=b_{\alpha}$. One can easily check that for $v, v^{\prime} \in \mathbb{F}_{4}^{*}$ with $v \neq v^{\prime}$ there exists a solution.

${ }^{13}$ See http://brauer.maths.qmul.ac.uk/Atlas/v3/misc/M20/.
} 


\section{Remarks:}

1. Here is a slightly more conceptual description of the group $\mathcal{G}_{4,4}$. It is included for the benefit of fussbudgets. The subgroup of the holomorph of the hexacode that preserves the first hexacode digit is the semidirect product $\mathcal{H}_{6}^{0}: F$. Its lift to $\mathrm{SU}(2)^{6}: S_{6}$ defines a subgroup $\widetilde{K}$ that fits in an exact sequence

$$
1 \rightarrow Z \rightarrow \widetilde{K} \rightarrow \mathcal{H}_{6}^{0}: F \rightarrow 0
$$

because, as we have seen, the lift of elements in $\left\langle g_{0}, \ldots, g_{5}\right\rangle$ act without central extension. When combining left-movers with right-movers we aim to produce a subgroup of $\operatorname{Spin}(4)^{6}: S_{6}$ where group elements that involve nontrivial permutations of factors must act diagonally on left- and right-movers. We therefore view $\operatorname{Spin}(4)^{6}: S_{6}$ as a fiber product of $\left(\mathrm{SU}(2)^{6}: S_{6}\right)_{L} \times_{p_{L}, p_{R}}\left(\mathrm{SU}(2)^{6}: S_{6}\right)_{R}$ where $p$ is the projection to $S_{6} \cdot{ }^{14}$ Our symmetry group will be a quotient of the fiber product $\widetilde{K}_{L} \times_{p_{L}, p_{R}} \widetilde{K}_{R}$. The reason we must take a quotient is that $Z_{L} \times Z_{R}$ does not act effectively on the GTVW spectrum. The reason is that

$$
\left(\epsilon_{1}, \ldots, \epsilon_{6}\right) \subset Z
$$

acts on $\mathcal{V}_{s}$ via the scalar $\prod_{\alpha} \epsilon_{\alpha}^{s_{\alpha}}$. But then the diagonally embedded subgroup $Z_{\text {diag }} \subset$ $Z_{L} \times Z_{R}$ acts on $\mathcal{V}_{s} \otimes \widetilde{\mathcal{V}}_{\tilde{s}}$ as

$$
\prod_{\alpha=1}^{6} \epsilon_{\alpha}^{s_{\alpha}+\tilde{s}_{\alpha}}
$$

If $\tilde{s}_{\alpha}=s_{\alpha}$ this factor is equal to 1 . If $\tilde{s}_{\alpha}=s_{\alpha}+1$ the factor is $\prod_{\alpha} \epsilon_{\alpha}=1$, by the definition of $Z$. One can check that $Z_{\text {diag }}$ is the largest subgroup of $Z_{L} \times Z_{R}$ that acts ineffectively. Thus, the group of symmetries preserving $(4,4)$ supersymmetry that we have identified is properly described as

$$
\mathcal{G}_{4,4} \cong\left(\widetilde{K}_{L} \times_{p_{L}, p_{R}} \widetilde{K}_{R}\right) / Z_{\operatorname{diag}}
$$

2. It is also instructive to compare our description of the generators of the group of $(4,4)$ preserving symmetries with the specific transformations studied in [41]. The $S_{6}$ elements (34)(56) and (35)(46) above correspond to the transformations $s_{v_{4}}$ and $s_{u}$ respectively in [41] and have a geometrical origin as half period shifts in the $T_{D_{4}} / \mathbb{Z}_{2}$ orbifold description of the GTVW sigma model. See their equation (4.34). The $S_{6}$ permutation (25)(34) corresponds to $\alpha^{p, T}$ and while less obvious is also a half-period shift. See their equation (4.62). This lifts to the element $\widehat{g}_{5}^{\prime}$ which is still an involution. Thus these elements generate a group isomorphic to $A_{5}$. The symmetries in equation (4.41) of [41] correspond to (the lift of) $\mathcal{H}_{6, L}^{0}$. Since they are purely left-moving they

\footnotetext{
${ }^{14}$ Recall that given groups and homomorphisms $\psi_{1}: G_{1} \rightarrow H$ and $\psi_{2}: G_{2} \rightarrow H$ the fiber product is

$$
G_{1} \times_{\psi_{1}, \psi_{2}} G_{2}=\left\{\left(g_{1}, g_{2}\right) \mid \psi_{1}\left(g_{1}\right)=\psi_{2}\left(g_{2}\right)\right\} .
$$
}


are nongeometric symmetries. See the discussion at the end of section 4.2 of [41]. Our group $Z$ corresponds to the symmetries denoted $t_{i} t_{j}$ in [41]. The symmetries $\gamma_{1}$ and $\gamma_{2}$, corresponding to rotations acting on the Kummer surface $T_{D_{4}} / \mathbb{Z}_{2}$ and the symmetries $s_{v_{1}+v_{2}}, s_{v_{2}+v_{4}}$ corresponding to or half period shifts in the $D_{4}$ lattice are related in equation (4.39) of [41] to diagonally embedded elements of $\mathcal{H}_{6}^{0}$. Our description is evidently less geometric, but has the benefit of unifying the treatment of symmetries in terms of the holomorph of the hexacode.

\subsubsection{Stabilizer of $(4,1)$ supersymmetry within $\operatorname{Spin}(4)^{6}: S_{6} / Z_{\text {diag }}$}

The ideas of section 5.4.1 can readily be generalized to produce a group of symmetries of the GTVW model that commute with $(4,1)$ supersymmetry. As discussed above, these should still be relevant to the degeneracies computed by the elliptic genus.

If we only aim to preserve a single $N=1$ right-moving supercurrent then we can drop the restriction that our symmetries act trivially on the first hexacode digit. Using the canonical description of (5.46) we replace $\widetilde{K}_{R}$ by the lift of the holomorph of the hexacode to $\operatorname{SU}(2)^{6}: S_{6}$. Specifically we now include elements $\varphi \in\left\langle g_{0}\right\rangle \times F \subset \operatorname{Aut}\left(\mathcal{H}_{6}\right)$ as well as arbitrary translations $w_{R} \in \mathcal{H}_{6}$. Call the lift $\widetilde{K}_{R}^{\prime}$. The analog of (5.46) is then

$$
\mathcal{G}_{4,1}=\left(\widetilde{K}_{L} \times_{p_{L}, p_{R}} \widetilde{K}_{R}^{\prime}\right) / Z_{\operatorname{diag}}
$$

The group will have structure analogous to (5.25):

$$
\mathcal{G}_{4,1} \cong Z \cdot\left(\left(\mathcal{H}_{6, L}^{0} \times \mathcal{H}_{6, R}\right): F\right)
$$

Note that we cannot make use of other automorphisms $\varphi_{R}$ in $\left\langle g_{1}, \ldots, g_{5}, g_{F}\right\rangle-F$ because these have permutations that change the first digit. Because of the fiber product structure (saying that the permutation image of the action of $\varphi$ on the left-movers and right-movers must be the same) such automorphisms of the CFT do not commute with the SU(2) Rsymmetry of the left-moving $\mathrm{N}=4$ superconformal algebra.

Note that $\mathcal{G}_{4,4}$ is a normal subgroup of $\mathcal{G}_{4,1}$ and

$$
\mathcal{G}_{4,1} / \mathcal{G}_{4,4} \cong\left(\mathcal{H}_{6, R} / \mathcal{H}_{6, R}^{0}\right):\left\langle\widehat{g}_{0}\right\rangle /\left\langle-1^{6}\right\rangle \cong\left(\mathbb{Z}_{2} \times \mathbb{Z}_{2}\right): \mathbb{Z}_{3}
$$

In this sense the group $\mathcal{G}_{4,1}$ is 12 times bigger. In particular,

$$
\left|\mathcal{G}_{4,1}\right|=2^{17} \cdot 3^{2} \cdot 5
$$

For comparison note that

$$
\left|M_{24}\right|=2^{10} \cdot 3^{3} \cdot 5 \cdot 7 \cdot 11 \cdot 23
$$

So, $\mathcal{G}_{4,1}$ cannot have $M_{24}$ as a quotient group, by Lagrange's theorem.

\subsection{The "new" twined elliptic genera}

In this section we define a few elliptic genera associated to some of the "new" elements in $\mathcal{G}_{4,1}$. We will not give a systematic study of the full vector space of such "new" elliptic genera. 
If $g$ is an automorphism in $\mathcal{G}_{4,1}$ we can define a twisted elliptic genus:

$$
\mathcal{E}_{g}(z, \tau):=\operatorname{Tr}_{\mathcal{H}_{R R}}(-1)^{F_{L}+F_{R}} U(g) e^{2 \pi \mathrm{i} z J_{0}} q^{H} \bar{q}^{\tilde{H}}
$$

where $J_{0}=2 J_{0}^{3}$ is normalized to have integral eigenvalues and $U(g)$ is the action on the GTVW space of states. The supersymmetric cancellations will continue to hold so we get a Jacobi form of weight zero and index 1 for a suitable congruence subgroup.

In order to compute (5.52) we begin by isolating the subspace of $\mathcal{H}_{R R}$ that contains R-moving groundstates, that is, the subspace of the GTVW state spacethat contains RR sector states with $(h, \tilde{h})=\left(h, \frac{1}{4}\right)$. To this end, for $1 \leq \alpha \leq 6$ define

$$
\mathcal{V}^{(\alpha)}=\mathcal{V}_{s_{1}} \otimes \cdots \mathcal{V}_{s_{6}}
$$

where $s_{\alpha}=1$ and $s_{\beta}=0$ for $\beta \neq \alpha$. Similarly, define

$$
\mathcal{V}^{(\alpha)+e}=\mathcal{V}_{s_{1}} \otimes \cdots \mathcal{V}_{s_{6}}
$$

where $s_{\alpha}=0$ and $s_{\beta}=1$ for $\beta \neq \alpha$. The relevant subspace of the RR states is then

$$
\oplus_{\alpha=1}^{6}\left[\mathcal{V}^{(\alpha)} \otimes \tilde{\mathcal{V}}^{(\alpha)} \oplus \mathcal{V}^{(\alpha)+e} \otimes \tilde{\mathcal{V}}^{(\alpha)}\right]
$$

Note that $(-1)^{F_{L}+F_{R}}$ is +1 on the first summand and -1 on the second summand in the expression in square brackets above.

Now, for a single Gaussian model we have

$$
\begin{aligned}
& \operatorname{Tr}_{\mathcal{V}_{0}} e^{2 \pi \mathrm{i} z J_{0}} q^{H}=\frac{\vartheta_{3}(2 z, 2 \tau)}{\eta(\tau)}:=f_{0}(z) \\
& \operatorname{Tr}_{\mathcal{V}_{1}} e^{2 \pi \mathrm{i} z J_{0}} q^{H}=\frac{\vartheta_{2}(2 z, 2 \tau)}{\eta(\tau)}:=f_{1}(z)
\end{aligned}
$$

also let $f_{0}:=f_{0}(0)$ and $f_{1}:=f_{1}(0)$. The twisted elliptic genera for group elements with $g^{L}=1$ are in the ring of functions of $(z, \tau)$ generated by $f_{0}(z), f_{1}(z), f_{0}, f_{1}$. In fact, they will be in the linear span of the functions:

$$
\begin{aligned}
& F_{0}(z, \tau):=f_{1}(z) f_{0}^{5}-f_{0}(z) f_{1}^{5} \\
& F_{1}(z, \tau):=f_{0}(z) f_{1} f_{0}^{4}-f_{1}(z) f_{0} f_{1}^{4}
\end{aligned}
$$

For example, the elliptic genus itself is just

$$
\mathcal{E}_{1}(z, \tau)=2\left(F_{0}+5 F_{1}\right)
$$

Now, for $x \in \mathbb{F}_{4}$ we choose coset representatives of $\mathcal{H}_{6} / \mathcal{H}_{6}^{0}$. We will make the explicit choice:

$$
\begin{aligned}
& w_{\underline{0}}:=(\underline{0}, \underline{0}, \underline{0}, \underline{0}, \underline{0}, \underline{0}) \\
& w_{\underline{1}}:=(\underline{1}, \underline{1}, \underline{\omega}, \underline{\omega}, \underline{\bar{\omega}}, \underline{\bar{\omega}}) \\
& w_{\underline{\omega}}:=(\underline{\omega}, \underline{\omega}, \underline{\bar{\omega}}, \underline{\bar{\omega}}, \underline{1}, \underline{1}) \\
& w_{\underline{\omega}}:=(\underline{\bar{\omega}}, \underline{\bar{\omega}}, \underline{1}, \underline{1}, \underline{\omega}, \underline{\omega})
\end{aligned}
$$


For $a \in \mathbb{Z}_{6}$ and $x \in \mathbb{F}_{4}$ define:

$$
\mathcal{E}_{g}^{a, x}(z, \tau):=\mathcal{E}_{g \cdot\left(1 ; \hat{g}_{0}^{-a} h\left(w_{x}\right)\right)}(z, \tau)
$$

Note that $\mathcal{E}_{g}^{a+3, x}(z, \tau)=-\mathcal{E}_{g}^{a, x}(z, \tau)$. If we apply this formula to $g \in \mathcal{G}_{4,4}$ then we define, in principle, $12-1=11$ "new" elliptic genera for each of the "old" elliptic genera.

A small computation leads to the following table of "new" twisted elliptic genera for the case $g=1$ :

\begin{tabular}{|c|c|c|c|c|}
\hline & $x=\underline{0}$ & $x=\underline{1}$ & $x=\underline{\omega}$ & $x=\underline{\underline{\omega}}$ \\
\hline$a=0$ & $2\left(F_{0}+F_{1}\right)$ & 0 & $4 F_{1}$ & $4 F_{1}$ \\
\hline$a=1$ & $F_{0}-3 F_{1}$ & $-\left(F_{0}+5 F_{1}\right)$ & $-F_{0}-F_{1}$ & $-F_{0}-F_{1}$ \\
\hline$a=2$ & $-\left(F_{0}+5 F_{1}\right)$ & $-\left(F_{0}+5 F_{1}\right)$ & $-\left(F_{0}+5 F_{1}\right)$ & $-\left(F_{0}+5 F_{1}\right)$ \\
\hline
\end{tabular}

One might wonder whether the new group elements we have found are related to a subquotient of the still-mysterious $M_{24}$ symmetry of K3 sigma models. We will argue now that they are not. Recall the discussion near equation (4.6). The representations of $M_{24}, H_{0,0}, H_{0,1 / 2}, H_{n}$, with $n \geq 1$ have the property that, for every $g \in M_{24}$, the function $\hat{\phi}_{g}(z, \tau)$ defined in (4.7) transform as Jacobi forms - precisely analogous to those in (5.52), as if $g \in M_{24}$ acted on the $\mathrm{CFT} \mathcal{C}$ as a $(4,4)$-preserving automorphism. Moreover as noted in (4.8), for $g \in \mathcal{G}_{4,4}$ they indeed coincide.

The first few representations $H_{0,0}, H_{0,1 / 2}$ and $H_{n} \quad n \geq 1$ determined by $[11,35,36$, $39,45]$ are

$$
\begin{aligned}
H_{0,0} & =\mathbf{2 3}-3 \cdot \mathbf{1} \\
H_{0,1 / 2} & =-2 \cdot \mathbf{1} \\
H_{1} & =\mathbf{4 5} \oplus \mathbf{4 5}^{*} \\
H_{2} & =\mathbf{2 3 1} \oplus \mathbf{2 3 1}^{*} \\
H_{3} & =\mathbf{7 7 0} \oplus \mathbf{7 7 0}^{*}
\end{aligned}
$$

We now explain that the twined genera associated to the "new" elements in $\mathcal{G}_{4,1}-\mathcal{G}_{4,4}$ do not conform to the expectation (4.8) by looking at a few examples. We begin by noting the decomposition of $F_{0}$ and $F_{1}$ into $N=4$ characters:

$$
\begin{aligned}
& F_{0}=-\chi_{0,1 / 2}-15 \chi_{5 / 4}-49 \chi_{9 / 4}-210 \chi_{13 / 4}-543 \chi_{17 / 4}-1484 \chi_{21 / 4}+\cdots \\
& F_{1}=2 \chi_{0,0}+12 \chi_{5 / 4}+56 \chi_{9 / 4}+196 \chi_{13 / 4}+564 \chi_{17 / 4}+1456 \chi_{21 / 4}
\end{aligned}
$$

1. Now note that for $g=\left(1 ;\left(\Omega^{\otimes 6}\right)^{a} h\left(w_{x}+(001111)\right)\right)$ we find $-\left(F_{0}+5 F_{1}\right)$. If our "new" $g$ were an $M_{24}$ element then we would need to have $\operatorname{Tr}_{H_{n}}(g)=-\frac{1}{2} \operatorname{dim} H_{n}$. There is no such group element.

2. Next consider $a=0, x=\underline{\omega}$ and $a=0, x=\underline{\bar{\omega}}$. These give $4 F_{1}$. Looking at (5.64) we see the coefficient of $c h_{0,1 / 2}$ is zero. On the other hand, $H_{0,1 / 2}=-21$ and the character of every element $g$ in this representation is -2 . This shows we have not made some error by confusing traces with supertraces. 
3. In some discussions of Moonshine authors will distinguish massless and massive states. Let us consider again the character $4 F_{1}$. We would need to find a $g \in M_{24}$ with

$$
\begin{aligned}
\operatorname{Tr}_{\mathbf{4 5} \oplus \mathbf{4 5}} *(g) & =4 \cdot 12 \\
\operatorname{Tr}_{\mathbf{2 3 1} \oplus \mathbf{2 3 1}^{*}}(g) & =4 \cdot 56
\end{aligned}
$$

it is easy to see from the character table of $M_{24}$ that no such $g$ exists.

4. Similarly, for $F_{0}-3 F_{1}$ we would require, at the massive level

$$
\begin{aligned}
\operatorname{Tr}_{\mathbf{4 5} \oplus \mathbf{4 5}} *(g) & =-51 \\
\operatorname{Tr}_{\mathbf{2 3 1} \oplus \mathbf{2 3 1}^{*}}(g) & =-217
\end{aligned}
$$

Again, no such $g$ exists.

\subsection{Stabilizer of an $N=1$ supercurrent within a group that includes parity- reversing operations}

As we will see in the next section, the full structure of the Golay code as a symmetry of the RR groundstates of the GTVW model only becomes apparent when we combine left- and right-moving supersymmetries and study the stabilizer, within $\operatorname{Pin}(4)^{6}: S_{6}$ of the $N=1$ supercurrent based on

$$
\Psi_{L}-\Psi_{R}
$$

The potential supercharges live in the subspace of the GTVW space of states:

$$
\mathcal{H}_{\mathrm{GTVW}}^{3 / 2,0} \oplus \mathcal{H}_{\mathrm{GTVW}}^{(0,3 / 2)} \cong \otimes_{\alpha=1}^{6}(\mathbf{2})_{L}^{(\alpha)} \oplus \otimes_{\alpha=1}^{6}(\mathbf{2})_{R}^{(\alpha)}
$$

In order to see the full symmetry of the RR states we need to extend the quaternion group $Q^{6}$ used in section 5.1 to include parity. We do this by extending $\mathrm{SU}(2)_{L} \times \mathrm{SU}(2)_{R}$ to Pin(4) and viewing Pin(4) $\subset \mathbb{H}(2)$, the algebra of $2 \times 2$ matrices over the quaternions. Viewed this way we are lead to consider a group consisting of elements

$$
\left(\left(\begin{array}{cc}
\epsilon_{L}^{1} h\left(x_{1}\right) & 0 \\
0 & \epsilon_{R}^{1} h\left(x_{1}\right)
\end{array}\right), \ldots,\left(\begin{array}{cc}
\epsilon_{L}^{6} h\left(x_{6}\right) & 0 \\
0 & \epsilon_{R}^{6} h\left(x_{6}\right)
\end{array}\right)\right)
$$

together with

$$
\left(\left(\begin{array}{cc}
0 & \epsilon_{L}^{1} h\left(x_{1}\right) \\
\epsilon_{R}^{1} h\left(x_{1}\right) & 0
\end{array}\right), \ldots,\left(\begin{array}{cc}
0 & \epsilon_{L}^{6} h\left(x_{6}\right) \\
\epsilon_{R}^{6} h\left(x_{6}\right) & 0
\end{array}\right)\right)
$$

where $x_{i} \in \mathbb{F}_{4}$. This is a non-abelian group of order $2^{25}$ and we denote it by $\widehat{Q}_{P}^{6}$. The subscript $P$ indicates that we have included a diagonally-acting parity operation. The above group acts naturally on $\mathcal{H}_{\mathrm{GTVW}}^{3 / 2,0} \oplus \mathcal{H}_{\mathrm{GTVW}}^{0,3 / 2}$. The stabilizer of the $N=1$ supercurrent 
determined by $\Psi_{L}-\Psi_{R}$ within $\widehat{Q}_{P}^{6}$ can be determined to be:

$$
\begin{aligned}
& \operatorname{Stab}_{\widehat{Q}_{P}^{6}}\left(\Psi_{L}-\Psi_{R}\right)=\left\{\left(\left(\begin{array}{cc}
\epsilon_{L}^{1} h\left(x_{1}\right) & 0 \\
0 & \epsilon_{R}^{1} h\left(x_{1}\right)
\end{array}\right), \ldots,\left(\begin{array}{cc}
\epsilon_{L}^{6} h\left(x_{6}\right) & 0 \\
0 & \epsilon_{R}^{6} h\left(x_{6}\right)
\end{array}\right)\right)\right. \\
& \left.\mid \prod_{i=1}^{6} \epsilon_{L}^{i}=1 \quad \& \quad \prod_{i=1}^{6} \epsilon_{R}^{i}=1 \quad \& \quad\left(x_{1}, \ldots, x_{6}\right) \in \mathcal{H}_{6}\right\} \\
& \amalg\left\{\left(\left(\begin{array}{cc}
0 & \epsilon_{L}^{1} h\left(x_{1}\right) \\
\epsilon_{R}^{1} h\left(x_{1}\right) & 0
\end{array}\right), \ldots,\left(\begin{array}{cc}
0 & \epsilon_{L}^{6} h\left(x_{6}\right) \\
\epsilon_{R}^{6} h\left(x_{6}\right) & 0
\end{array}\right)\right)\right. \\
& \left.\mid \prod_{i=1}^{6} \epsilon_{L}^{i}=-1 \quad \& \quad \prod_{i=1}^{6} \epsilon_{R}^{i}=-1 \quad \& \quad\left(x_{1}, \ldots, x_{6}\right) \in \mathcal{H}_{6}\right\}
\end{aligned}
$$

This is a non-abelian group of order $2^{5} \times 2^{5} \times 4^{3} \times 2$. The main significance of this group will be apparent when we consider its action on the RR ground states.

We should note that above we have only discussed the "translation symmetries" of $\Psi_{L}-\Psi_{R}$. There will also be a larger group making use of the "rotational" automorphisms of the hexacode. We have not explored this larger symmetry in detail.

\section{RR states and the MOG construction of the Golay code}

In this section we will show that the group (5.73) that preserves the $N=1$ supercurrent $\Psi_{L}-\Psi_{R}$ also acts on the space of RR ground states according to a pattern governed by the Golay code. The pattern emerges when we use a special basis of RR states, so we be begin by explaining this distinguished basis.

The space of RR ground states, as an $\left(\mathrm{SU}(2)_{L} \times \mathrm{SU}(2)_{R}\right)^{6}$ representation, has the structure:

$$
\mathcal{V}_{R R}=\mathcal{H}_{\mathrm{GTVW}}^{1 / 4,1 / 4} \cong \oplus_{\alpha=1}^{6}(\mathbf{2} ; \mathbf{2})^{(\alpha)}
$$

Now the representation $(\mathbf{2} ; \mathbf{2})$ of $\mathrm{SU}(2)_{L} \times \mathrm{SU}(2)_{R}$ admits a canonical real structure and the resulting four-dimensional real vector space, as a representation of $\mathrm{SU}(2) \times \mathrm{SU}(2)$ can be identified with the quaternions, as a representation of $U(1, \mathbb{H}) \times U(1, \mathbb{H})$. The resulting canonical basis is:

$$
\begin{aligned}
& |1\rangle=\frac{1}{\sqrt{2}}(|+-\rangle-|-+\rangle) \\
& |2\rangle=\frac{1}{\sqrt{2}}(|++\rangle+|--\rangle) \\
& |3\rangle=\frac{\mathrm{i}}{\sqrt{2}}(|++\rangle-|--\rangle) \\
& |4\rangle=\frac{\mathrm{i}}{\sqrt{2}}(|+-\rangle+|-+\rangle)
\end{aligned}
$$

This slightly peculiar basis of states appeared in [41]. It is determined by the quaternionic structure and in particular is compatible with the real structure. Readers interested in understanding the above remarks more thoroughly can consult appendix D. 
The Pauli matrices $h(x)$ when acting under the diagonal embedding $\rho_{L R}(h(x))=$ $(h(x), h(x))$ will act diagonally on this basis, with entries \pm 1 along the diagonal. We will represent such a matrix by a column vector with entries 0,1 . We convert $+\leftrightarrow 0$ and $-\leftrightarrow 1$. Thus, for example, $(h(\underline{1}), h(\underline{1}))$ acts in this basis as the diagonal matrix

$$
\left(\begin{array}{llll}
+ & & \\
& + & \\
& & - & \\
& & & -
\end{array}\right)
$$

and we summarize that action by the column vector

$$
\left(\begin{array}{l}
0 \\
0 \\
1 \\
1
\end{array}\right)
$$

In this way, the signs appearing in the action of $\rho_{L R}(h(x))$, for $x \in \mathbb{F}_{4}$, on the canonical basis of $\mathbb{H} \cong((1 / 2) \otimes(1 / 2))_{\mathbb{R}}$ are neatly encoded in the map $g^{+}(x, 0)$ defined in equation (2.43).

Next, when we act with the operators $(h(w), h(w))$, for $w \in \mathbb{F}_{4}^{6}$ on the distinguished basis of the RR sector we obtain a $4 \times 6$ array of elements of $\mathbb{F}_{2}$. This array can, in turn, be identified with a vector in $\mathbb{F}_{2}^{24}$.

The action of $\widehat{Q}_{P}^{6}$ on $\mathcal{H}_{\mathrm{GTVW}}^{1 / 4,1 / 4}$ factors through to an action of an Abelian group

$$
\left(\mathbb{F}_{4}^{+} \times \mathbb{F}_{2}\right)^{6} \times \mathbb{Z}_{2}
$$

Acting on $\mathcal{H}_{\text {GTVW }}^{1 / 4,1 / 4}$ in the distinguished basis we find an action of the Golay code - in the following sense.

Group elements of the form

$$
\left(\left(\begin{array}{cc}
\epsilon_{L}^{1} h\left(x_{1}\right) & 0 \\
0 & \epsilon_{R}^{1} h\left(x_{1}\right)
\end{array}\right), \ldots,\left(\begin{array}{cc}
\epsilon_{L}^{6} h\left(x_{6}\right) & 0 \\
0 & \epsilon_{R}^{6} h\left(x_{6}\right)
\end{array}\right)\right)
$$

acts on the distinguished basis of RR states as:

$$
V^{i, \alpha} \rightarrow(-1)^{g^{+}\left(x_{\alpha}, \epsilon_{L}^{\alpha} \epsilon_{R}^{\alpha}\right)_{i}} V^{i, \alpha}
$$

while group elements

$$
\left(\left(\begin{array}{cc}
0 & \epsilon_{L}^{1} h\left(x_{1}\right) \\
\epsilon_{R}^{1} h\left(x_{1}\right) & 0
\end{array}\right), \ldots,\left(\begin{array}{cc}
0 & \epsilon_{L}^{6} h\left(x_{6}\right) \\
\epsilon_{R}^{6} h\left(x_{6}\right) & 0
\end{array}\right)\right)
$$

acts as

$$
V^{i, \alpha} \rightarrow(-1)^{g^{-}\left(x_{\alpha}, \epsilon_{L}^{\alpha} \epsilon_{R}^{\alpha}\right)_{i}} V^{i, \alpha}
$$

Comparing with the description of the Golay code in equations (2.48) to (2.53) we arrive at one of our main statements: 
Consider the stabilizer of $\Psi_{L}-\Psi_{R}$ within a left-right symmetric version of the quaternion group (or group of error operators), namely the group $\widehat{Q}_{P}^{6}$ defined above. This stabilizer group is a non-Abelian group which, when acting on $V_{R R}$ in the distinguished basis defined by the $\mathrm{SU}(2)^{6}$ WZW model and the quaternions, defines the Golay code. In equations, there is a natural isomorphism

$$
\mathcal{G} \cong \rho_{V_{R R}}\left(\operatorname{Stab}_{\widehat{Q}_{P}^{6}}\left(\Psi_{L}-\Psi_{R}\right)\right)
$$

This gives a physical interpretation of the MOG presentation of the Golay code. Note that, if we do not consider the extension by parity, and only consider the left-rightsymmetric action of the group preserving $\Psi$ then we obtain the even Golay code $\mathcal{G}^{+}$.

What is the significance of this result? It is well-known that the automorphism group of the Golay code is $M_{24}$. This then, gives a new interpretation of an $M_{24}$ "symmetry" within a K3 sigma model. We put "symmetry" in quotation marks because it is an automorphism group of a symmetry group. It is not clear to us what implication such a "symmetry of a group of symmetries" has for twined elliptic genera and the space of massive BPS states. It is possible that the emerging ideas in the context of "generalized symmetries" and domain walls will shed further light on this question.

There are two natural directions in which the above could be extended: first, we have once again left off any investigation of the role of the "rotational" symmetries $\operatorname{Aut}\left(\mathcal{H}_{6}\right)$ in the holomorph of the hexacode. Second, we limited discussion to the left-right symmetric action on the canonical RR basis. One could of course, also consider the same group elements acting either on the right or on the left. The action will then be by signed permutation matrices.

\section{Superconformal symmetry and QEC in Conway moonshine}

The methods of this paper shed some light on the Conway Moonshine module studied in $[28,29]$ since very similar techniques can be used to construct the $N=1$ supercurrent that plays a starring role in the analysis of those papers. We are merely making concrete and explicit some points left implicit in [28, 29].

The Conway Moonshine module can be thought of as a theory of 24 Majorana-Weyl spinors $\psi_{i}, i=1, \ldots, 24$. This theory has a $\operatorname{Spin}(24)$ automorphism group. In the Ramond sector the ground states form a $2^{12}$ dimensional represention of $\operatorname{Spin}(24)$ with a natural real structure. The vertex operators associated to these states have conformal dimension $h=24 \times \frac{1}{16}=\frac{3}{2}$, and therefore there is a $2^{12}$ dimensional space of potential supercurrents $V_{s}$ labeled by $\operatorname{Spin}(24)$ spinors $s$. Using the representation theory of the Conway group [28, 29] showed that there is a distinguished spinor $s$ such that $V_{s}$ indeed defines a superconformal current, and moreover, the stabilizer of $\operatorname{Spin}(24)$ acting on $s$ is exactly the largest sporadic Conway group, thus identifying the Conway group as a group of $N=1$ supersymmetry preserving automorphisms. What we will do here is construct the spinor explicitly using the Golay code and show, in an elementary way, how the properties of the Golay code imply the required identities for $V_{s}$ to define a supercurrent. 
The OPE of $V_{s}$ with itself is given by [101]

$$
V_{s}(1) V_{s}(2) \sim \frac{s^{t r} s}{z_{12}^{3}}+\frac{s^{t r} \gamma^{i j} s}{z_{12}^{2}} \psi_{i} \psi_{j}+\frac{s^{t r} s}{z_{12}} T+\frac{s^{t r} \gamma^{i j k l} s}{z_{12}} \psi_{i} \psi_{j} \psi_{k} \psi_{l}+\cdots
$$

This follows from Spin(24) symmetry. Therefore, the OPE of $V_{s}$ with itself will define a supercurrent provided

$$
\begin{array}{cc}
s^{t r} \gamma^{i j} s=0 & i<j \\
s^{t r} \gamma^{i j k l} s=0 & i<j<k<l
\end{array}
$$

We will now construct a solution to these equations. In the Ramond sector the zeromodes of $\psi_{i}$ will be 24 Clifford generators $\gamma_{i}$ with $\gamma_{i}^{2}=1$. Now, for $w \in \mathbb{F}_{2}^{24}$ let

$$
\gamma_{w}:=\gamma_{1}^{w_{1}} \cdots \gamma_{24}^{w_{24}}
$$

Then

$$
\gamma_{w_{1}} \gamma_{w_{2}}=\epsilon\left(w_{1}, w_{2}\right) \gamma_{w_{1}+w_{2}}
$$

where

$$
\epsilon\left(w_{1}, w_{2}\right)=(-1)^{\sum_{i<j} w_{2}^{i} w_{1}^{j}}
$$

is a nontrivial cocycle. Indeed, it describes the Heisenberg extension of $\mathbb{F}_{2}^{24}$ by $\mathbb{F}_{2}$. The key observation is that, when restricted to the Golay code $\mathcal{G} \subset \mathbb{F}_{2}^{24}$ the cocycle is trivializable by a \pm 1 -valued coboundary.

We will use the MOG presentation to represent Golay code words as $\mathfrak{f}^{+}(x, \epsilon)$ or $\mathfrak{f}^{-}(x, \epsilon)$ where $(x, \epsilon)$ is a decorated hexacode word as described between equations (2.48) to (2.53). Label the basis vectors and gamma matrices by 1,2,3,4 going from top to bottom in the first column, 5, 6, 7, 8 top to bottom in the second column, etc.

Then, we let, for example:

$$
\begin{aligned}
& \gamma_{g^{+}(\underline{0}, 0)}=1 \\
& \gamma_{g^{+}(\underline{0}, 1)}=\gamma_{1} \gamma_{2} \gamma_{3} \gamma_{4}=\gamma_{1234} \\
& \gamma_{g^{+}(\underline{1}, 0)}=\gamma_{3} \gamma_{4}=\gamma_{34} \\
& \gamma_{g^{+}(\underline{1}, 1)}=\gamma_{1} \gamma_{2}=\gamma_{12} \\
& \gamma_{g^{+}(\underline{\omega}, 0)}=\gamma_{2} \gamma_{4}=\gamma_{24} \\
& \gamma_{g^{+}(\underline{\bar{\omega}}, 0)}=\gamma_{2} \gamma_{2}=\gamma_{23}
\end{aligned}
$$

Now, if $w=\left(\left(x_{1}, \epsilon_{1}\right), \ldots,\left(x_{6}, \epsilon_{6}\right)\right)=(x, \epsilon)$ is a decorated hexacode word we let

$$
\gamma_{\mathfrak{f}^{+}(w)}:=\gamma_{g+\left(x_{1}, \epsilon_{1}\right)}^{(1)} \cdots \gamma_{g+\left(x_{6}, \epsilon_{6}\right)}^{(6)}
$$

where the superscript in parentheses indicates which quartet of gamma matrices we are using. For $\gamma_{g+\left(x_{1}, \epsilon_{1}\right)}^{(1)}$ we use the set of four $\gamma$-matrices $\gamma_{1}, \ldots, \gamma_{4}$ for $\gamma_{g+\left(x_{2}, \epsilon_{2}\right)}^{(2)}$ we use the set of four $\gamma$-matrices $\gamma_{5}, \ldots, \gamma_{8}$, and so on.

A computation shows that if $w \in \mathcal{G}$ corresponds to $(x, \epsilon)$ and we define

$$
b(w)=(-1)^{\sum_{\alpha} \delta\left(x_{\alpha}\right) \epsilon_{\alpha}}
$$


where, for $x_{\alpha} \in \mathbb{F}_{4}$,

$$
\delta\left(x_{\alpha}\right):= \begin{cases}0 & x_{\alpha}=\underline{0}, \underline{\omega} \\ 1 & x_{\alpha}=\underline{1}, \underline{\bar{\omega}}\end{cases}
$$

then, for $w, w^{\prime} \in \mathcal{G}$ we have

$$
\gamma_{w} \gamma_{w^{\prime}}=\frac{b\left(w+w^{\prime}\right)}{b(w) b\left(w^{\prime}\right)} \gamma_{w+w^{\prime}}
$$

It therefore follows that if we define $\tilde{\gamma}_{w}:=b(w) \gamma_{w}$ then

$$
P_{D}:=\frac{1}{2^{12}} \sum_{w \in \mathcal{G}} \tilde{\gamma}_{w}
$$

is a rank one projection operator. Duncan's spinor is in the image of this projection operator: $s_{D} \propto P s_{0}$ for any generic spinor $s_{0}$.

It is now elementary to explain why equations (7.2) and (7.3) are satisfied by Duncan's spinor. We observe that

$$
s_{D} s_{D}^{t r}=k P_{D}
$$

for a suitable constant $k$. (We are working in a real vector space $\mathbb{R}^{2^{12}}$ with Euclidean norm.) Therefore

$$
\begin{aligned}
s_{D}^{t r} \gamma_{i j} s_{D} & =\operatorname{Tr} \gamma_{i j} s_{D} s_{D}^{t r} \\
& =k \operatorname{Tr} \gamma_{i j} P_{D} \\
& =\frac{k}{2^{12}} \sum_{w \in \mathcal{G}} b(w) \operatorname{Tr} \gamma_{i j} \gamma_{w}
\end{aligned}
$$

and similarly for $s_{D}^{t r} \gamma_{i j k l} s_{D}$. Next note that

$$
\begin{aligned}
\gamma_{i j} \gamma_{w} & = \pm \gamma_{w+e_{i}+e_{j}} \\
\gamma_{i j k l} \gamma_{w} & = \pm \gamma_{w+e_{i}+e_{j}+e_{k}+e_{l}}
\end{aligned}
$$

but, thanks to the error-correcting properties of the Golay code, this means that $w+e_{i}+e_{j}$ and $w+e_{i}+e_{j}+e_{k}+e_{l}$ are never Golay code words. Thus, $\operatorname{Tr} \gamma_{i j} \gamma_{w}=0$ for all Golay code words $w$ and $i<j$, and similarly $\operatorname{Tr} \gamma_{i j k l} \gamma_{w}=0$. The proof is thus closely analogous to our proof that $V_{\Psi}$ generates a superconformal current in the GTVW model.

An argument closely analogous to that of section 5.3 shows that the stabilizer group of the image of $P$ is a finite subgroup of $\operatorname{Spin}(24)$. It is easy to show from the above description of the projection operator that the stabilizer of Duncan's spinor contains the maximal subgroup $2^{12}: M_{24}$ of $\mathrm{Co}_{0}$. The fact that the stabilizer is exactly $\mathrm{Co}_{0}$ is more nontrivial and follows from general arguments in $[28,29]$. It would be very nice to demonstrate this directly using the above description for the projection operator. Such a demonstration appears to be nontrivial, and this will be left for another occasion. 


\subsection{Relation between conway and GTVW superconformal currents}

There is a close relation between the, so-called "reflected GTVW model" and the Conway Moonshine module explored in [19, 96]. Starting with the Conway Moonshine module, we split the 24 fermions according to the MOG: $\psi_{\alpha}^{a}$, with $a=1, \ldots, 4$. For each $\alpha$ we have a $\mathfrak{s o}(4) \cong \mathfrak{s u}(2)_{\alpha}^{L} \oplus \mathfrak{s u}(2)_{\alpha}^{R} k=(1,1)$ current algebra. We then have six copies of the VOA's these generate corresponding to the six columns of the MOG and we identify that as a subalgebra of the reflected GTVW theory. Now the reflected GTVW theory has an $N=1$ superconformal current ${ }^{15}$

$$
V_{\Psi_{L}}+V_{\Psi_{R}}
$$

with $c=12$ and energy-momentum tensor

$$
T=-\frac{1}{2} \psi_{\alpha}^{a} \partial \psi_{\alpha}^{a}
$$

Given the uniqueness of the $N=1$ structure $V_{\Psi_{L}}+V_{\Psi_{R}}$ should coincide with $V_{s_{\text {Duncan }}}$ up to automorphism of the CFT. We can see this rather nicely as follows: for any pair $\left(\alpha_{1}, \alpha_{2}\right)$ of columns in the MOG consider the decorated hexacode word $(x, \epsilon)$ with $x_{\alpha}=\underline{0}$ for all $\alpha$ and

$$
\epsilon_{\alpha}= \begin{cases}0 & \alpha \neq \alpha_{1}, \alpha_{2} \\ 1 & \alpha \in\left\{\alpha_{1}, \alpha_{2}\right\}\end{cases}
$$

Then $\mathfrak{f}^{+}(x, \epsilon)$ is an even interpretation of the zero hexacode word, the trivialization $b(x, \epsilon)=$ 1 and

$$
\tilde{\gamma}_{\mathfrak{f}^{+}(x, \epsilon)}
$$

is just the product of the column chirality operators for columns $\alpha_{1}$ and $\alpha_{2}$. Thus, $s_{D}$ can be expressed as $s^{+}+s^{-}$where $s^{+}$has all column chiralities equal to +1 and $s^{-}$has all column chiralities equal to -1 .

Now note that if we decompose the spinor representation of $\mathfrak{s o}(24)$ under the subalgebra

$$
\oplus_{\alpha} \mathfrak{s o}(4)_{\alpha}
$$

then we get:

$$
((\mathbf{2} ; 0) \oplus \widetilde{(0 ; \mathbf{2})})_{\alpha=1} \otimes \cdots((\mathbf{2} ; 0) \oplus \widetilde{(0 ; \mathbf{2})})_{\alpha=6} \cong \oplus_{x+\tilde{x}=e} V_{x} \otimes \tilde{V}_{\tilde{x}}
$$

where $V_{0}$ is the singlet of $\mathfrak{s u}(2)$ and $V_{1}$ is the doublet of $\mathfrak{s u}(2)$ and $e$ is the all ones vector. The GTVW supercurents live in the components

$$
\begin{aligned}
& V_{\Psi_{L}} \in V_{1^{6}} \otimes \tilde{V}_{0^{6}} \\
& V_{\Psi_{R}} \in V_{0^{6}} \otimes \tilde{V}_{1^{6}}
\end{aligned}
$$

but $V_{1^{6}} \otimes \tilde{V}_{0^{6}}$ is the image of the projection operators:

$$
P_{+}=\left(\frac{1+\gamma^{1234}}{2}\right)_{\alpha=1} \otimes \cdots \otimes\left(\frac{1+\gamma^{1234}}{2}\right)_{\alpha=6}
$$

\footnotetext{
${ }^{15}$ Indeed, one take the reflected sum of each of the four supercurrents leading to an $N=4$ structure on the reflected GTVW model [96].
} 
onto the space of spinors with all column chiralities $=+1$ and $V_{0^{6}} \otimes \tilde{V}_{1^{6}}$ is the image of the projection operator

$$
P_{-}=\left(\frac{1-\gamma^{1234}}{2}\right)_{\alpha=1} \otimes \cdots \otimes\left(\frac{1-\gamma^{1234}}{2}\right)_{\alpha=6}
$$

with all column chiralities $=-1$. This shows that

$$
\begin{aligned}
& V_{\Psi_{L}}=s^{+} \\
& V_{\Psi_{R}}=s^{-}
\end{aligned}
$$

We can write:

$$
P_{D}=2^{-12}\left(\sum_{w \in \mathcal{G}^{+}} \tilde{\gamma}_{\mathfrak{f}^{+}}+\sum_{w \in \mathcal{G}^{-}} \tilde{\gamma}_{\mathfrak{f}^{-}}\right)
$$

The first term preserves all column chiralities and the second term changes all column chiralities. The first term can be restricted to $V_{1^{6}} \otimes \tilde{V}_{0^{6}}$, and this should coincide with $P_{\text {GTVW }}$.

\section{A The automorphism group of the hexacode and the even Golay code}

In this paper we adopt notation for finite groups and their extensions used in [5]. In particular, $p^{m}$ indicates the group $(\mathbb{Z} / p \mathbb{Z})^{m}, A \times B$ is the direct product of the groups $A$ and $B, A . B$ indicates a group with normal subgroup $A$ and quotient isomorphic to $B$ while $A: B$ denotes a group which is a semi-direct product of $A$ and $B$. Of course for the latter a full description requires specifying a homomorphism $\phi: B \rightarrow \operatorname{Aut}(A)$.

For any group $G$ we define the holomorph of $G$ to be the group $\operatorname{Hol}(G):=G: \operatorname{Aut}(G)$ where the semi-direct product is defined using the natural action of $\operatorname{Aut}(G)$ on $G$. Thus, the group $\operatorname{Hol}(G)$ acts naturally on $G$ itself where the first factor acts by (say) left-translation. A good example is the group of automorphisms of real $n$-dimensional affine space, which is isomorphic to the holomorph of the group $\mathbb{R}^{n}$.

The hexacode has some very useful symmetries, and in this appendix we review the structure of it automorphism group $\operatorname{Aut}\left(\mathcal{H}_{6}\right)$ in some detail.

First of all, being a linear subspace of $\mathbb{F}_{4}^{6}$, a hexacode word is mapped to another by multiplication by any scalar, and if the scalar is nonzero this is an automorphism. We denote the group of scalar multiplication by nonzero elements of $\mathbb{F}_{4}$ by $H_{0}$. Of course $H_{0} \cong \mathbb{Z}_{3}$ and one generator would be

$$
g_{0}:\left(x_{1}, \ldots, x_{6}\right) \rightarrow\left(\underline{\omega} x_{1}, \ldots, \underline{\omega} x_{6}\right)
$$

Next, there are some simple permutation symmetries, i.e. subgroups of the natural $S_{6}$ action on $\mathbb{F}_{4}^{6}$ that preserve $\mathcal{H}_{6}$. To describe these it is useful to arrange a 6-digit word in $\mathcal{H}_{6}$ as 3 couples: $\left(x_{1}, \ldots, x_{6}\right)=(a b c d$ ef $)$. One such group of symmetries is obtained by flipping pairs of couples. We can take as generators:

$$
\begin{aligned}
& g_{1}=(12)(34) \\
& g_{2}=(34)(56)
\end{aligned}
$$


these generate a group $H_{1} \cong \mathbb{Z}_{2} \times \mathbb{Z}_{2}$. Another subgroup of permutation symmetries is obtained by arbitrary permutation of couples. This defines a subgroup $H_{2} \cong S_{3}$. One choice of generators of $\mathrm{H}_{2}$ would be:

$$
\begin{aligned}
& g_{3}=(13)(24) \\
& g_{4}=(35)(46)
\end{aligned}
$$

Clearly $H_{2}$ normalizes $H_{1}$ and together these generate a group $H_{3}=H_{1}: H_{2} \cong S_{4}$. One way to prove that $H_{3}$ is a group of symmetries of the hexacode proceeds by applying the generators $g_{1}, \ldots, g_{4}$ to three basis vectors in equation (2.29) and checking that the resulting vectors remain in the hexacode. Together with scalar multiplication we obtain a subgroup $H_{0} \times H_{3} \cong \mathbb{Z}_{3} \times S_{4}$ of the automorphism group. One can check directly that the orbit of the four "seed codewords"

$$
(\underline{11} \underline{\omega \omega} \underline{\bar{\omega} \bar{\omega}}) \quad(\underline{00} \underline{11} \underline{11}) \quad(\underline{\omega \bar{\omega}} \underline{\omega \bar{\omega}} \underline{\omega \bar{\omega}}) \quad(\underline{01} \underline{01} \underline{\omega \bar{\omega}})
$$

under $H_{0} \times H_{3}$ is the entire set of nonzero words in the hexacode. See eq. (A.14).

There are further, "nonobvious" automorphisms of the hexacode. An example of such an automorphism is

$$
g_{5}:\left(x_{1}, \ldots, x_{6}\right) \rightarrow\left(\underline{\omega} x_{1}, \underline{\bar{\omega}} x_{2}, x_{3}, x_{6}, x_{4}, x_{5}\right)
$$

To prove that $g_{5}$ is a symmetry note that $\left(x_{1}, \ldots, x_{6}\right) \in \mathcal{H}_{6}$ iff

$$
\begin{aligned}
& x_{4}=\Phi_{x_{1}, x_{2}, x_{3}}(\underline{1})=x_{1}+x_{2}+x_{3} \\
& x_{5}=\Phi_{x_{1}, x_{2}, x_{3}}(\underline{\omega})=\underline{\omega} x_{1}+\underline{\omega} x_{2}+x_{3} \\
& x_{6}=\Phi_{x_{1}, x_{2}, x_{3}}(\underline{\bar{\omega}})=\underline{\omega} x_{1}+\underline{\bar{\omega}} x_{2}+x_{3}
\end{aligned}
$$

(See equation (2.28) above.) Then, letting $g_{5} \cdot\left(x_{1}, \ldots, x_{6}\right)=\left(y_{1}, \ldots y_{6}\right)$,

$$
\begin{aligned}
& y_{4}=\Phi_{y_{1}, y_{2}, y_{3}}(\underline{1})=y_{1}+y_{2}+y_{3}=\underline{\omega} x_{1}+\underline{\bar{\omega}} x_{2}+x_{3}=x_{6} \\
& y_{5}=\Phi_{y_{1}, y_{2}, y_{3}}(\underline{\omega})=\underline{\bar{\omega}} y_{1}+\underline{\omega} y_{2}+y_{3}=x_{1}+x_{2}+x_{3}=x_{4} \\
& y_{6}=\Phi_{y_{1}, y_{2}, y_{3}}(\underline{\bar{\omega}})=\underline{\omega} y_{1}+\underline{\bar{\omega}} y_{2}+y_{3}=\underline{\bar{\omega}} x_{1}+\underline{\omega} x_{2}+x_{3}=x_{5}
\end{aligned}
$$

Let $H_{5}:=\left\langle g_{1}, \ldots, g_{5}\right\rangle$. There is a projection

$$
p: \operatorname{Aut}\left(\mathcal{H}_{6}\right) \rightarrow S_{6}
$$

where the image of $p$ just tracks how the automorphism permutes the hexacode digits. Clearly, the kernel of $p$ is just $H_{0}$. Moreover, one can check by direct computation that the image of $H_{5}$ under $p$ is the entire subgroup $A_{6} \subset S_{6}$. Thus, $H_{05}:=\left\langle g_{0}, \ldots, g_{5}\right\rangle$ is a central extension of $A_{6}$ by $H_{0} \cong \mathbb{Z}_{3}$. By computing the lift of two elements in $A_{6}$ whose group commutator vanishes one easily checks that it is a nontrivial central extension, so ${ }^{16}$

$$
H_{05}:=\left\langle g_{0}, \ldots, g_{5}\right\rangle \cong \mathbb{Z}_{3} \cdot A_{6}
$$

\footnotetext{
${ }^{16}$ For a nice discussion see Lecture one of [68].
} 
Another example of a non-obvious automorphism of $\mathcal{H}_{6}$ is

$$
g_{F}:\left(x_{1}, \ldots, x_{6}\right)=(56) \cdot\left(x_{1}^{2}, \ldots, x_{6}^{2}\right)=(56) \cdot\left(\bar{x}_{1}, \ldots, \bar{x}_{6}\right)
$$

In the second equality we have used the fact that the nonlinear map $x \rightarrow x^{2}$ is identical to the Frobenius automorphism $x \rightarrow \bar{x}$ of $\mathbb{F}_{4}$. To prove that $g_{F}$ is an automorphism of the hexacode we again use equation (2.28) and the result follows immediately. Since $p\left(g_{F}\right)=(56)$ is an odd permutation it is clear that the image under $p$ of $\left\langle g_{1}, \ldots, g_{5}, g_{F}\right\rangle$ is all of $S_{6}$. On the other hand, $g_{F}$ does not commute with $g_{0}$ and conjugation by $g_{F}$ acts as the nontrivial automorphism of $H_{0}$. Thus,

$$
\left\langle g_{0}, g_{1}, \ldots, g_{5}, g_{F}\right\rangle \cong H_{0} \cdot\left\langle g_{1}, \ldots, g_{5}, g_{F}\right\rangle \cong \mathbb{Z}_{3} \cdot S_{6} .
$$

In [99] it is asserted that the full automorphism group $\operatorname{Aut}\left(\mathcal{H}_{6}\right) \cong \mathbb{Z}_{3} \cdot S_{6}$ so we have now described in detail the full structure of the automorphism group of the hexacode.

In computations it can be useful to have a full list of hexacode words. We provide this list in eq. (A.14) which contains all hexacode words with the exception of the trivial word 000000 . The words are organized into orbits of $H_{2} \cong S_{3}$ and $H_{1} \cong \mathbb{Z}_{2} \times \mathbb{Z}_{2}$ generated by $g_{1}, g_{2}$.

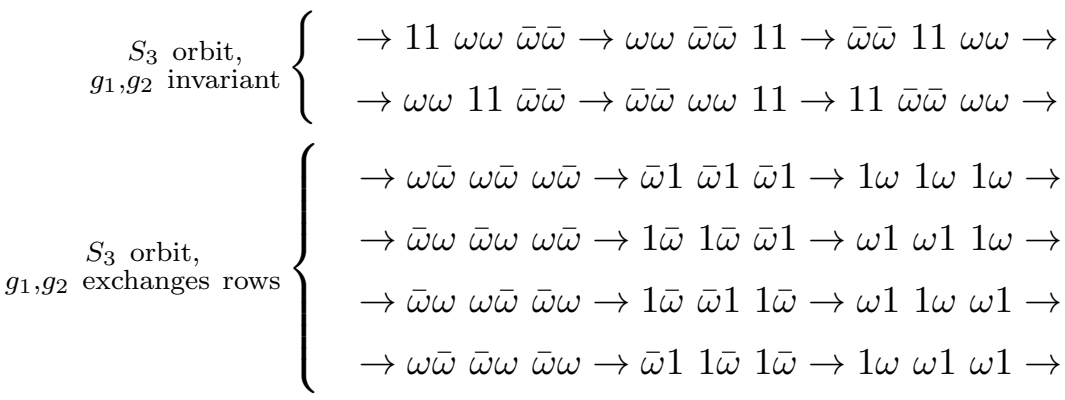

$$
\begin{aligned}
& \underset{g_{1}, g_{2} \text { invariant }}{S_{3} \text { orbit, }}\left\{\begin{array}{l}
\rightarrow 001111 \rightarrow 00 \omega \omega \omega \omega \rightarrow 00 \bar{\omega} \bar{\omega} \bar{\omega} \bar{\omega} \rightarrow \\
\rightarrow 110011 \rightarrow \omega \omega 00 \omega \omega \rightarrow \bar{\omega} \bar{\omega} 00 \bar{\omega} \bar{\omega} \rightarrow \\
\rightarrow 111100 \rightarrow \omega \omega \omega \omega 00 \rightarrow \bar{\omega} \bar{\omega} \bar{\omega} \bar{\omega} 00 \rightarrow
\end{array}\right.
\end{aligned}
$$

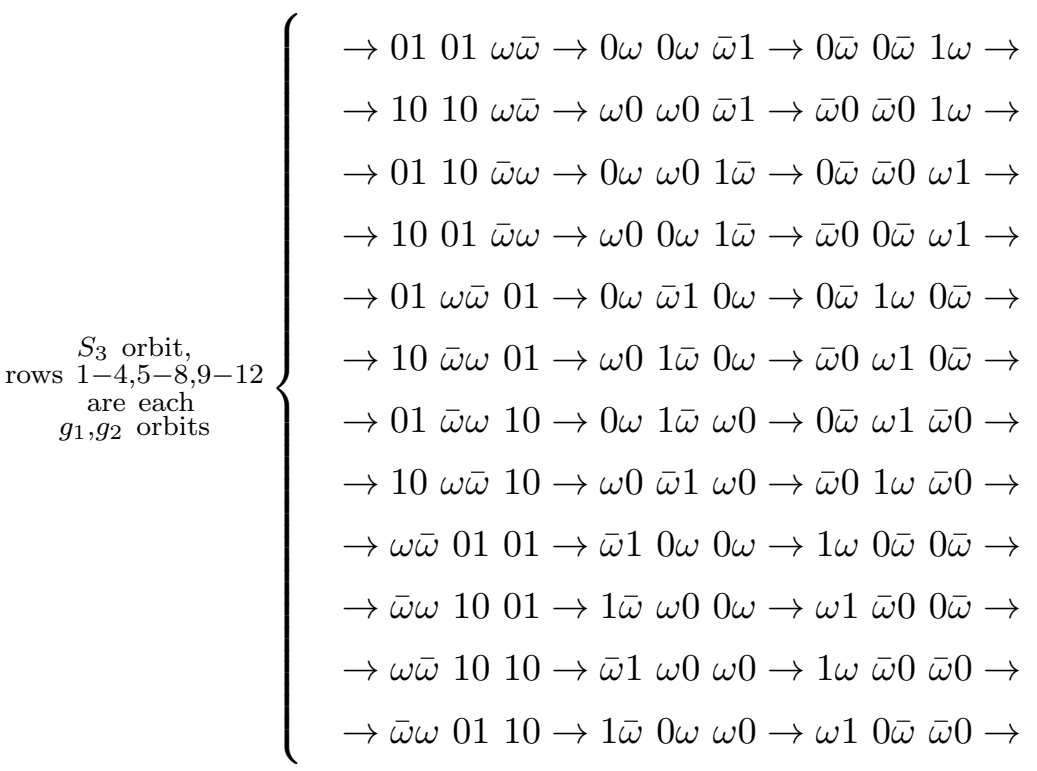


Remark: it is quite interesting to note that the automorphisms of the hexacode act on two distinct sets of six objects. The first is the set of six digits. However, the automorphisms also permutes the no-zeroes hexacode words (i.e. those words where none of the digits is zero). Up to overall scale there are exactly six types of no-zeroes hexacode words. They correspond to the first six lines of (A.14). The relation between these two group actions of $S_{6}$ on sets with six elements is related by an exceptional outer automorphism of the symmetric group $S_{6}$.

\section{B Supersymmetry conventions}

\section{B.1 Superconformal algebras}

The $N=12$ d superconformal algebra has generators $G_{r}, L_{m}$ with commutation relations

$$
\begin{aligned}
\left\{G_{r}, G_{s}\right\} & =2 L_{r+s}+\frac{c}{12}\left(4 r^{2}-1\right) \delta_{r+s, 0} \\
{\left[L_{m}, G_{r}\right] } & =\left(\frac{m}{2}-r\right) G_{m+r} \\
{\left[L_{m}, L_{n}\right] } & =(m-n) L_{m+n}+\frac{c}{12}\left(m^{3}-m\right) \delta_{m+n, 0}
\end{aligned}
$$

Here $m \in \mathbb{Z}$ and $r \in \mathbb{Z}$ or $r \in \mathbb{Z}+\frac{1}{2}$ for the Ramond or Neveu-Schwarz algebra respectively. In terms of OPE's of the currents:

$$
\begin{aligned}
& T(z)=\sum_{n} z^{-n-2} L_{n} \\
& G(z)=\sum_{r} z^{-r-3 / 2} G_{r}
\end{aligned}
$$

we have

$$
\begin{aligned}
& G(z) G(w) \sim \frac{\frac{2 c}{3}}{(z-w)^{3}}+\frac{\frac{1}{2} T(w)}{z-w}+\cdots \\
& T(z) G(w) \sim \frac{\frac{3}{2} G(w)}{(z-w)^{2}}+\frac{\partial G(w)}{z-w}+\cdots \\
& T(z) T(w) \sim \frac{\frac{c}{2}}{(z-w)^{4}}+\frac{2 T(w)}{(z-w)^{2}}+\frac{\partial T(w)}{z-w}+\cdots
\end{aligned}
$$


The small $N=4$ superconformal algebra has generators $L_{n}, Q_{r}^{a}, T_{m}^{i}$ with relations:

$$
\begin{aligned}
{\left[L_{m}, L_{n}\right] } & =(m-n) L_{m+n}+\frac{k}{2}\left(m^{3}-m\right) \delta_{m+n, 0} \\
\left\{Q_{r}^{a}, Q_{s}^{b}\right\} & =\left\{\bar{Q}_{r}^{a}, \bar{Q}_{s}^{b}\right\}=0 \\
\left\{Q_{r}^{a}, \bar{Q}_{s}^{b}\right\} & =2 \delta^{a b} L_{r+s}-2(r-s) \sigma_{a b}^{i} T_{r+s}^{i}+\frac{k}{2}\left(4 r^{2}-1\right) \delta_{r+s, 0} \delta^{a b} \\
{\left[T_{m}^{i}, T_{n}^{j}\right] } & =i \epsilon^{i j k} T_{m+n}^{k}+\frac{k}{2} m \delta_{m+n, 0} \delta^{i j} \\
{\left[T_{m}^{i}, Q_{r}^{a}\right] } & =-\frac{1}{2} \sigma_{a b}^{i} Q_{m+r}^{b} \\
{\left[L_{m}, Q_{r}^{a}\right] } & \left.=\left(\frac{m}{2}-r\right) Q_{m+r}^{a}, \bar{Q}_{r}^{a}\right]=\frac{1}{2}\left(\sigma_{a b}^{i}\right)^{*} \bar{Q}_{m+r}^{b} \\
{\left[L_{m}, T_{n}^{i}\right] } & =-n T_{m+n}^{i}
\end{aligned}
$$

Here it is traditional to parametrize by $c=6 k$ and $k \in \mathbb{Z}_{+}$for unitary theories. Here we are using the conventions of $[33,34]$. There is a natural real structure on this algebra defined by

$$
\left(Q_{r}^{a}\right)^{\dagger}=\bar{Q}_{-r}^{a} \quad\left(T_{n}^{i}\right)^{\dagger}=T_{-n}^{i}
$$

Note that since the $T_{0}^{i}$ are real they will be represented by Hermitian operators in a unitary representation whereas in our conventions $J_{0}^{i}$ are antihermitian.

As explained above we are interested in embeddings of the $N=1$ superconformal algebra into the $N=4$ algebra. We thus require that a linear combination of $N=4$ supercurrents

$$
G_{r}=\alpha_{1} Q_{r}^{1}+\alpha_{2} Q_{r}^{2}+\beta_{1} \bar{Q}_{r}^{1}+\beta_{2} \bar{Q}_{r}^{2}
$$

satisfies the $N=1$ superconformal algebra. Moreover, we require that under the real structure (B.5) we have $\left(G_{r}\right)^{\dagger}=G_{-r}$. It is not difficult to show that, up to an overall $\mathrm{SU}(2)_{R}$ rotation the most general solution is

$$
G=\frac{1}{2}\left(Q^{1}+\bar{Q}^{1}\right)
$$

Note that this linear combination is not an eigenstate of $T_{0}^{3}$. In fact, this is a vector in the $\mathbf{2} \oplus \mathbf{2}$ that completely breaks continuous $\mathrm{SU}(2)_{R}$-symmetry.

To make contact with the notation used in [41] we note that

$$
Q^{1} \propto G^{+} \quad \bar{Q}^{1} \propto G^{-} \quad \bar{Q}^{2} \propto G^{\prime} \quad Q^{2} \propto G^{\prime-}
$$

Since $\left(G^{+}, G^{\prime},-\right)$ is an $\mathrm{SU}(2)_{R}$ doublet and $\left(G^{\prime},+, G^{-}\right)$is another $\mathrm{SU}(2)_{R}$ doublet.

\section{Proof that there are no nonzero solutions of (5.18)}

To show that (5.18) has no nontrivial solutions we write

$$
\mathcal{O}:=\sum_{x \in \mathbb{F}_{4}^{*}} \sum_{\alpha=1}^{6} c_{x, \alpha} h(x)_{\alpha}
$$


As a sum

$$
\begin{aligned}
\mathcal{O} & =\mathcal{O}_{\text {diag }}+\mathcal{O}_{\text {flip }} \\
\mathcal{O}_{\text {diag }} & :=\sum_{\alpha=1}^{6} c_{\underline{\bar{\omega}}, \alpha} h(\underline{\bar{\omega}})_{\alpha} \\
\mathcal{O}_{\text {flip }} & :=\sum_{\alpha=1}^{6}\left(c_{\underline{1}, \alpha} h(\underline{1})_{\alpha}+c_{\underline{\omega}, \alpha} h(\underline{\omega})_{\alpha}\right)
\end{aligned}
$$

The idea here is that $\mathcal{O}_{\text {diag }}$ acts on the spin states in $\Psi$ just by multiplying by a phase, while $\mathcal{O}_{\text {flip }}$ flips one digit. So these terms cannot interfere.

So, setting $\mathcal{O}_{\text {diag }} \Psi=0$ we get 16 equations. For example, acting on $[\emptyset]$ we get

$$
-\mathrm{i}\left(c_{\underline{\underline{\omega}}, 1}+c_{\underline{\bar{\omega}}, 2}+\cdots+c_{\underline{\bar{\omega}}, 6}\right)=0
$$

while acting on [12] we get:

$$
\mathrm{i}\left(c_{\underline{\bar{\omega}}, 1}+c_{\underline{\bar{\omega}}, 2}\right)-\mathrm{i}\left(c_{\underline{\bar{\omega}}, 3}+c_{\underline{\bar{\omega}}, 4}+c_{\underline{\bar{\omega}}, 5}+c_{\underline{\bar{\omega}}, 6}\right)=0
$$

Altogether we 16 equations. They are not all independent but we find independent equations

$$
\begin{aligned}
& c_{\underline{\bar{\omega}}, 1}+c_{\underline{\bar{\omega}}, 2}+\cdots+c_{\underline{\bar{\omega}}, 6}=0 \\
& \left(c_{\underline{\bar{\omega}}, 1}+c_{\underline{\bar{\omega}}, 2}\right)-\left(c_{\underline{\bar{\omega}}, 3}+c_{\underline{\bar{\omega}}, 4}+c_{\underline{\bar{\omega}}, 5}+c_{\underline{\bar{\omega}}, 6}\right)=0 \\
& \left(c_{\underline{\bar{\omega}}, 3}+c_{\underline{\bar{\omega}}, 4}\right)-\left(c_{\underline{\bar{\omega}}, 1}+c_{\underline{\omega}, 2}+c_{\underline{\bar{\omega}}, 5}+c_{\underline{\bar{\omega}}, 6}\right)=0 \\
& \left(c_{\underline{\bar{\omega}}, 5}+c_{\underline{\bar{\omega}}, 6}\right)-\left(c_{\underline{\bar{\omega}}, 1}+c_{\underline{\bar{\omega}}, 2}+c_{\underline{\bar{\omega}}, 3}+c_{\underline{\bar{\omega}}, 4}\right)=0 \\
& \left(c_{\underline{\bar{\omega}}, 1}+c_{\underline{\bar{\omega}}, 3}+c_{\underline{\bar{\omega}}, 5}\right)-\left(c_{\underline{\bar{\omega}}, 2}+c_{\underline{\bar{\omega}}, 4}+c_{\underline{\bar{\omega}}, 6}\right)=0 \\
& \left(c_{\underline{\bar{\omega}}, 2}+c_{\underline{\bar{\omega}}, 4}+c_{\underline{\bar{\omega}}, 5}\right)-\left(c_{\underline{\bar{\omega}}, 1}+c_{\underline{\bar{\omega}}, 3}+c_{\underline{\bar{\omega}}, 6}\right)=0 \\
& \left(c_{\underline{\bar{\omega}}, 1}+c_{\underline{\bar{\omega}}, 4}+c_{\underline{\bar{\omega}}, 6}\right)-\left(c_{\underline{\bar{\omega}}, 2}+c_{\underline{\bar{\omega}}, 3}+c_{\underline{\bar{\omega}}, 5}\right)=0
\end{aligned}
$$

Some of the tedium of writing these equations can be reduced by recalling that $\Psi$ is symmetric under permutations of the couples (12), (34), (56), and this symmetry must be reflected in the equations. In any case, by adding and subtracting equations we quickly find that $c_{\underline{\bar{\omega}}, 6}=-c_{\underline{\bar{\omega}}, 5}, c_{\overline{\bar{\omega}}, 4}=-c_{\overline{\bar{\omega}}, 3}$ and $c_{\underline{\bar{\omega}}, 2}=-c_{\underline{\bar{\omega}}, 1}$ and

$$
\begin{aligned}
& c_{\overline{\underline{\omega}}, 1}+c_{\overline{\underline{\omega}}, 3}+c_{\overline{\underline{\omega}}, 5}=0 \\
& c_{\underline{\bar{\omega}}, 2}+c_{\underline{\bar{\omega}}, 4}+c_{\underline{\bar{\omega}}, 5}=0 \\
& c_{\overline{\underline{\omega}}, 1}+c_{\overline{\underline{\omega}}, 4}+c_{\overline{\underline{\omega}}, 6}=0
\end{aligned}
$$

The determinant of the $3 \times 3$ matrix is nonzero and hence $c_{\overline{\underline{\omega}}, a}=0$.

Similarly, with the bit-flip operator $\mathcal{O}_{\text {flip }}$ note that $[\emptyset]$ is mapped to states $[a]$ with just one minus sign. While states like [12] are mapped to states with just one or just three minus signs. The equations split nicely. For example the coefficients of the following 
output spin states give us the following equations:

$$
\begin{aligned}
{[1] } & -c_{\underline{1}, 1}+\mathrm{i} c_{\underline{\omega}, 1}+\mathrm{i} c_{\underline{1}, 2}-c_{\underline{\omega}, 2}=0 \\
{[2] } & -c_{\underline{1}, 2}+\mathrm{i} c_{\underline{\omega}, 2}+\mathrm{i} c_{\underline{1}, 1}-c_{\underline{\omega}, 1}=0 \\
{[156] } & -\mathrm{i} c_{\underline{1}, 1}-c_{\underline{\omega}, 1}+c_{\underline{1}, 2}+\mathrm{i} c_{\underline{\omega}, 2}=0 \\
{[256] } & -\mathrm{i} c_{\underline{1}, 2}-c_{\underline{\omega}, 2}+c_{\underline{1}, 1}+\mathrm{i} c_{\underline{\omega}, 1}=0
\end{aligned}
$$

Computing the determinant of the relevant $4 \times 4$ matrix we find it is nonzero and hence

$$
c_{\underline{1}, 1}=c_{\underline{\omega}, 1}=c_{\underline{1}, 2}=c_{\underline{\omega}, 2}=0
$$

Taking into account the symmetry of the permutations of couples, we conclude that the identical equations hold for the couple (34) and for (56) and hence all the $c_{x, a}=0$. It follows that the stabilizer group is a discrete subgroup of $\mathrm{SU}(2)^{6}$.

\section{Quaternions give a distinguished basis for the $(2 ; 2)$ representation of $\mathrm{SU}(2) \times \mathrm{SU}(2)$}

Let $U(\mathbb{H})$ denote the group of unit quaternions. There is a standard representation:

$$
T: U(\mathbb{H}) \times U(\mathbb{H}) \longrightarrow \mathrm{GL}(\mathbb{H})
$$

defined by

$$
T\left(q_{1}, q_{2}\right): q_{0} \rightarrow q_{1} q_{0} q_{2}^{-1}
$$

Here $G L(\mathbb{H})$ is the group of invertible linear transformations regarding $\mathbb{H}$ as a real vector space.

Now let $\mathfrak{i}, \mathfrak{j}, \mathfrak{k}$ be unit quaternions, and use the choice of matrices $h(x)$ in this paper to define a map

$$
\mathbb{H} \rightarrow \operatorname{Mat}_{2 \times 2}(\mathbb{C})
$$

by taking

$$
\begin{aligned}
1 \rightarrow h(\underline{0}) & :=\left(\begin{array}{ll}
1 & 0 \\
0 & 1
\end{array}\right) \\
\mathfrak{i} \rightarrow h(\underline{1}) & :=\left(\begin{array}{cc}
0 & 1 \\
-1 & 0
\end{array}\right) \\
\mathfrak{j} \rightarrow h(\underline{\omega}) & :=\left(\begin{array}{ll}
0 & \mathrm{i} \\
\mathrm{i} & 0
\end{array}\right) \\
\mathfrak{k} \rightarrow h(\underline{\bar{\omega}}) & :=\left(\begin{array}{cc}
-\mathrm{i} & 0 \\
0 & \mathrm{i}
\end{array}\right)
\end{aligned}
$$

and extending $\mathbb{R}$-linearly. Restricted to $U(\mathbb{H})$ this defines a group isomorphism:

$$
U(\mathbb{H}) \stackrel{\psi}{\longrightarrow} \mathrm{SU}(2)
$$


On the other hand, we can define a real representation of $\mathrm{SU}(2) \times \mathrm{SU}(2)$

$$
R: \mathrm{SU}(2) \times \mathrm{SU}(2) \longrightarrow \mathrm{GL}(V)
$$

where $V$ is a real four-dimensional vector space and $\mathrm{GL}(V)$ is the group of real invertible linear transformations on $V$. This is just the $(2 ; 2)$ representation with a reality condition. Explicitly, letting $\alpha, \dot{\beta}$ run over $\{+,-\}$ in that order vectors in $V$ can be written as

$$
X_{\alpha, \dot{\beta}}|\alpha, \dot{\beta}\rangle
$$

where we impose the reality condition:

$$
\left(X_{\alpha \dot{\beta}}\right)^{*}=\epsilon^{\alpha \alpha^{\prime}} \epsilon^{\dot{\beta} \dot{\beta}^{\prime}} X_{\alpha^{\prime}, \dot{\beta}^{\prime}}
$$

The general solution of this reality condition is:

$$
X_{\alpha \dot{\beta}}=\left(\begin{array}{cc}
z & -\bar{w} \\
w & \bar{z}
\end{array}\right)
$$

where $z, w \in \mathbb{C}$. In these terms the $\mathrm{SU}(2) \times \mathrm{SU}(2)$ representation is:

$$
R\left(u_{1}, u_{2}\right)|\alpha ; \dot{\beta}\rangle=\left(u_{1}\right)_{\alpha^{\prime} \alpha}\left(u_{2}\right)_{\dot{\beta}^{\prime}, \dot{\beta}}^{*}\left|\alpha^{\prime} ; \dot{\beta}^{\prime}\right\rangle
$$

and in terms of $X_{\alpha \dot{\beta}}$ this is

$$
X_{\alpha \dot{\beta}} \rightarrow\left(u_{1} X u_{2}^{-1}\right)_{\alpha \dot{\beta}}
$$

Now we claim there is a unique isomorphism of real vector spaces

$$
\varphi: \mathbb{H} \rightarrow V
$$

such that

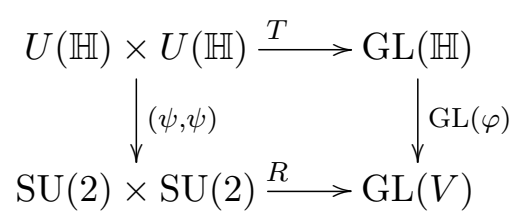

commutes. Here $\operatorname{GL}(\varphi)$ is the group isomorphism induced by the isomorphism of real vector spaces.

Using this distinguished isomorphism we define

$$
\begin{aligned}
\varphi(1) & =|1\rangle \\
\varphi(\mathfrak{i}) & =|2\rangle \\
\varphi(\mathfrak{j}) & =-|3\rangle \\
\varphi(\mathfrak{k}) & =-|4\rangle
\end{aligned}
$$

The basis $\{|1\rangle,|2\rangle,|3\rangle,|4\rangle\}$ is that given above in equation (6.2) above. Note the signs in the last two equations. So, the basis is not that canonical, but the difference does not affect the way the Golay code appears. 
Open Access. This article is distributed under the terms of the Creative Commons Attribution License (CC-BY 4.0), which permits any use, distribution and reproduction in any medium, provided the original author(s) and source are credited.

\section{References}

[1] L. Álvarez-Gaumé et al., Bosonization in arbitrary genus, Phys. Lett. B 178 (1986) 41 [INSPIRE].

[2] L. Álvarez-Gaumé et al., Bosonization on higher genus Riemann surfaces, Commun. Math. Phys. 112 (1987) 503 [INSPIRE].

[3] P.S. Aspinwall and D.R. Morrison, String theory on K3 surfaces, hep-th/9404151 [INSPIRE].

[4] P.S. Aspinwall, K3 surfaces and string duality, hep-th/9611137 [INSPIRE].

[5] J.H. Conway et al., Atlas of finite groups, Clarendon Press, Oxford U.K. (1985).

[6] http://brauer.maths.qmul.ac.uk/Atlas/v3/permrep/2Co1G1-p196560B0

[7] T. Beth and M. Grassl, The quantum hamming and hexacodes, Fortschr. Phys. 46 (1998) 459.

[8] D. Belov and G.W. Moore, Holographic Action for the Self-Dual Field, hep-th/0605038 [INSPIRE].

[9] R. Borcherds, Monstrous moonshine and monstrous Lie superalgebras, Invent. Math. 109 (1992) 405.

[10] P. Candelas, G.T. Horowitz, A. Strominger and E. Witten, Vacuum configurations for superstrings, Nucl. Phys. B 258 (1985) 46 [InSPIRE].

[11] M.C.N. Cheng, K3 surfaces, $N=4$ dyons and the Mathieu group M24, Commun. Num. Theor. Phys. 4 (2010) 623 [arXiv: 1005.5415] [INSPIRE].

[12] M.C.N. Cheng, J.F.R. Duncan and J.A. Harvey, Umbral Moonshine, Commun. Num. Theor. Phys. 08 (2014) 101 [arXiv: 1204.2779] [InSPIRE].

[13] M.C.N. Cheng, J.F.R. Duncan and J.A. Harvey, Umbral Moonshine and the Niemeier lattices, arXiv:1307.5793 [INSPIRE].

[14] M.C.N. Cheng, S.M. Harrison, R. Volpato and M. Zimet, K3 string theory, lattices and Moonshine, arXiv:1612.04404 [INSPIRE].

[15] V. Anagiannis and M.C.N. Cheng, TASI lectures on Moonshine, PoS(TASI 2017) 010.

[16] J.H. Conway, A group of order 8,315, 553,613,086,720,000, Bull. London Math. Soc. 1 (1969) 79.

[17] J.H. Conway and S.P. Norton, Monstrous Moonshine, Bull. London Math. Soc. 11 (1979) 308.

[18] J.H. Conway and S.P. Norton, Sphere packings, lattices and groups, Grundlehren der mathematischen Wissenschaften volume 290, Springer, Germany (1999).

[19] T. Creutzig, J.F.R. Duncan and W. Riedler, Self-dual vertex operator superalgebras and superconformal field theory, J. Phys. A 51 (2018) 034001 [arXiv:1704.03678] [INSPIRE]. 
[20] R.T. Curtis, A new combinatorial approach to $M_{24}$, Proc. Cambridge Phil. Soc. 79 (1976) 25.

[21] L.J. Dixon, P.H. Ginsparg and J.A. Harvey, Beauty and the beast: superconformal symmetry in a Monster module, Commun. Math. Phys. 119 (1988) 221 [InSPIRE].

[22] L.J. Dixon, P.H. Ginsparg and J.A. Harvey, (central charge c) = 1 superconformal field theory, Nucl. Phys. B 306 (1988) 470 [InSPIRE].

[23] L. Dolan, P. Goddard and P. Montague, Conformal field theory, triality and the Monster group, Phys. Lett. B 236 (1990) 165 [INSPIRE].

[24] C. Dong, G. Mason and Y. Zhu, Discrete series of the Virasoro algebra and the Moonshine module, Proc. Symp. Pure Math. 56 (1994) 295.

[25] C. Dong, R.L. Griess Jr. and G. Hohn, Framed vertex operator algebras, codes and the Moonshine module, Commun. Math. Phys. 193 (1998) 407.

[26] M.R. Douglas and S. Kachru, Flux compactification, Rev. Mod. Phys. 79 (2007) 733 [hep-th/0610102] [INSPIRE].

[27] M.R. Douglas and N.A. Nekrasov, Noncommutative field theory, Rev. Mod. Phys. 73 (2001) 977 [hep-th/0106048] [INSPIRE].

[28] J.F.R. Duncan, Super-Moonshine for Conway's largest sporadic group, Duke Math. J. 139 (2007) 255 .

[29] J.F.R. Duncan and S. Mack-Crane, The Moonshine module for Conway's group, SIGMA 3 (2015) e10 [arXiv:1409.3829] [INSPIRE].

[30] J.F.R. Duncan, M.J. Griffin and K. Ono, Proof of the umbral Moonshine conjecture, arXiv: 1503.01472 [INSPIRE].

[31] J.F.R. Duncan, M.H. Mertens and K. Ono, O'Nan moonshine and arithmetic, arXiv: 1702.03516.

[32] J.F.R. Duncan, M.J. Griffin and K. Ono, Moonshine, arXiv:1411.6571 [InSPIRE].

[33] T. Eguchi and A. Taormina, On the unitary representations of $N=2$ and $N=4$ superconformal algebras, Phys. Lett. B 210 (1988) 125 [INSPIRE].

[34] T. Eguchi, H. Ooguri, A. Taormina and S.-K. Yang, Superconformal algebras and string compactification on manifolds with $\mathrm{SU}(N)$ holonomy, Nucl. Phys. B 315 (1989) 193 [INSPIRE].

[35] T. Eguchi, H. Ooguri and Y. Tachikawa, Notes on the K3 surface and the Mathieu group $M_{24}$, Exper. Math. 20 (2011) 91 [arXiv:1004.0956] [INSPIRE].

[36] T. Eguchi and K. Hikami, Note on twisted elliptic genus of K3 surface, Phys. Lett. B 694 (2011) 446 [arXiv: 1008.4924] [INSPIRE].

[37] S. Ferrara, J.A. Harvey, A. Strominger and C. Vafa, Second quantized mirror symmetry, Phys. Lett. B 361 (1995) 59 [hep-th/9505162] [INSPIRE].

[38] I.B. Frenkel, J. Lepowsky and A. Meurman, A natural representation of the Fischer-Griess Monster with the modular function $J$ as character, Proc. Nat. Acad. Sci. U.S.A. 81 (1984) 3256 .

[39] M.R. Gaberdiel, S. Hohenegger and R. Volpato, Mathieu Moonshine in the elliptic genus of K3, JHEP 10 (2010) 062 [arXiv: 1008.3778] [INSPIRE]. 
[40] M.R. Gaberdiel, S. Hohenegger and R. Volpato, Symmetries of K3 $\sigma$-models, Commun. Num. Theor. Phys. 6 (2012) 1 [arXiv:1106.4315] [INSPIRE].

[41] M.R. Gaberdiel, A. Taormina, R. Volpato and K. Wendland, A K3 $\sigma$-model with $\mathbb{Z}_{2}^{8}: \mathbb{M}_{20}$ symmetry, JHEP 02 (2014) 022 [arXiv:1309.4127] [INSPIRE].

[42] M.R. Gaberdiel, C.A. Keller and H. Paul, Mathieu Moonshine and symmetry surfing, J. Phys. A 50 (2017) 474002 [arXiv: 1609.09302] [InSPIRE].

[43] D. Gaiotto and A. Kapustin, Spin TQFTs and fermionic phases of matter, Int. J. Mod. Phys. A 31 (2016) 1645044 [arXiv: 1505. 05856] [InSPIRE].

[44] D. Gaiotto and T. Johnson-Freyd, Holomorphic SCFTs with small index, arXiv: 1811.00589 [INSPIRE].

[45] T. Gannon, Much ado about Mathieu, Adv. Math. 301 (2016) 322 [arXiv:1211.5531] [INSPIRE].

[46] T. Gannon, Monstrous Moonshine: the first twenty five years, math/0402345.

[47] The GAP group, GAP - Groups, Algorithms, and Programming, version 4.8.8 (2017), https://www.gap-system.org.

[48] F. Gliozzi, J. Scherk and D.I. Olive, Supersymmetry, supergravity theories and the dual spinor model, Nucl. Phys. B 122 (1977) 253 [INSPIRE].

[49] P. Goddard, Meromorphic conformal field theory, in Infinite dimensional Lie algebras and Lie groups, V. Kac ed., World Scientific, Singapore (1989).

[50] D. Gottesman, An introduction to quantum error correction and fault-tolerant quantum computation, arXiv:0904.2557.

[51] M.B. Green and J.H. Schwarz, Anomaly cancellation in supersymmetric d $=10$ gauge theory and superstring theory, Phys. Lett. B 149 (1984) 117.

[52] M.J. Griffin and M. Mertens, A proof of the Thompson Moonshine conjecture, arXiv: 1607.03078.

[53] D.J. Gross, J.A. Harvey, E.J. Martinec and R. Rohm, The heterotic string, Phys. Rev. Lett. 54 (1985) 502 [INSPIRE].

[54] D.J. Gross, J.A. Harvey, E.J. Martinec and R. Rohm, Heterotic string theory. 1. The free heterotic string, Nucl. Phys. B 256 (1985) 253 [INSPIRE].

[55] D.J. Gross, J.A. Harvey, E.J. Martinec and R. Rohm, Heterotic string theory. 2. The interacting heterotic string, Nucl. Phys. B 267 (1986) 75 [INSPIRE].

[56] J.A. Harvey and G.W. Moore, Algebras, BPS states and strings, Nucl. Phys. B 463 (1996) 315 [hep-th/9510182] [INSPIRE].

[57] J.A. Harvey and G.W. Moore, On the algebras of BPS states, Commun. Math. Phys. 197 (1998) 489 [hep-th/9609017] [INSPIRE].

[58] J.A. Harvey and G.W. Moore, An uplifting discussion of T-duality, JHEP 05 (2018) 145 [arXiv: 1707.08888] [INSPIRE].

[59] J.A. Harvey and G.W. Moore, Conway subgroup symmetric compactifications of heterotic string, J. Phys. A 51 (2018) 354001 [arXiv:1712.07986] [InSPIRE].

[60] J.A. Harvey and B.C. Rayhaun, Traces of singular moduli and moonshine for the Thompson group, Commun. Num. Theor. Phys. 10 (2016) 23 [arXiv:1504.08179] [InSPIRE]. 
[61] G. Hoehn and G. Mason, The 290 fixed-point sublattices of the Leech lattice, J. Algebra 448 (2016) 618 [arXiv: 1505.06420] [INSPIRE].

[62] P.S. Howe and G. Papadopoulos, Ultraviolet behavior of two-dimensional supersymmetric nonlinear $\sigma$ models, Nucl. Phys. B 289 (1987) 264 [INSPIRE].

[63] C.M. Hull, Lectures on nonlinear sigma models and strings, in the proceedings of NATO Advanced Research Workshop on Super Field Theory, July 25-August 5, Vancouver, Canada (1986).

[64] C. Hull and U. Lindström, All $(4,1)$ : $\sigma$-models with $(4, q)$ off-shell supersymmetry, JHEP 03 (2017) 042 [arXiv:1611.09884] [INSPIRE].

[65] C. Hull and U. Lindström, The generalised complex geometry of $(p, q)$ Hermitian geometries, Commun. Math. Phys. (2019) 1 [arXiv:1810.06489] [INSPIRE].

[66] D. Huybrechts, On derived categories of K3 surfaces and Mathieu groups, arXiv: 1309.6528 [INSPIRE].

[67] T. Johnson-Freyd, Supersymmetry and the Suzuki chain, arXiv:1908.11012 [INSPIRE].

[68] T. Johnson-Freyd, http://categorified.net/Mathcamp-SporadicGroupsClass.pdf

[69] N. Junla, Classification of certain genera of codes, lattices and vertex operator algebras, Ph.D. thesis, Kansas State University, U.S.A. (2014).

[70] S. Kachru, Elementary introduction to Moonshine, 2016, arXiv:1605.00697 [INSPIRE].

[71] S. Kachru and C. Vafa, Exact results for $N=2$ compactifications of heterotic strings, Nucl. Phys. B 450 (1995) 69 [hep-th/9505105] [INSPIRE].

[72] S. Kachru, N.M. Paquette and R. Volpato, 3D string theory and umbral Moonshine, J. Phys. A 50 (2017) 404003 [arXiv: 1603.07330] [inSPIRE].

[73] A. Kapustin and R. Thorngren, Fermionic SPT phases in higher dimensions and bosonization, JHEP 10 (2017) 080 [arXiv:1701.08264] [INSPIRE].

[74] A. Karch, D. Tong and C. Turner, A web of $2 d$ dualities: $\mathbb{Z}_{2}$ gauge fields and Arf invariants, SciPost Phys. 7 (2019) 007 [arXiv: 1902.05550] [INSPIRE].

[75] C.A. Keller and I.G. Zadeh, Lifting 1/4-BPS states on K3 and Mathieu Moonshine, arXiv:1905.00035 [INSPIRE].

[76] A. Konechny and A.S. Schwarz, Introduction to M(atrix) theory and noncommutative geometry, Phys. Rept. 360 (2002) 353 [hep-th/0012145] [INSPIRE].

[77] C.H. Lam, Codes and vertex operator algebras, Alg. Comb. 1109 (1999) 106.

[78] Y.-H. Lin and S.-H. Shao, Duality defect of the Monster CFT, arXiv:1911.00042 [INSPIRE].

[79] T. Mainiero, Homological tools for the quantum mechanic, arXiv:1901.02011 [INSPIRE].

[80] G. Mason, Symplectic automorphisms of K3-surfaces (after S. Mukai and V.V. Nikulin), CWI Newslett. No. 13 (1986).

[81] N.D. Mermin, Quantum computer science. An introduction, Cambridge University Press, Cambridge U.K. (2007).

[82] R. Miranda and D.R. Morrison, Embeddings of integral quadratic forms, http://www.math.ucsb.edu/ drm/manuscripts/eiqf.pdf 
[83] M. Miyamoto, Binary codes and vertex operator (super) algebras, J. Alg. 181 (1996) 207.

[84] G. Moore, lecture notes, http://www.physics.rutgers.edu/ gmoore/695Fall2015/TopologicalFieldTheory.pdf.

[85] W. Nahm and K. Wendland, A hiker's guide to K3: aspects of $N=(4,4)$ superconformal field theory with central charge $c=6$, Commun. Math. Phys. 216 (2001) 85 [hep-th/9912067] [INSPIRE].

[86] http://www.math.rwth-aachen.de/ Gabriele.Nebe/LATTICES/

[87] A. Neveu and J.H. Schwarz, Factorizable dual model of pions, Nucl. Phys. B 31 (1971) 86 [INSPIRE].

[88] M.A. Nielsen and I.L. Chuang, Quantum computation and quantum information, Cambridge University Press, Cambridge U.K. (2010).

[89] N.M. Paquette, D. Persson and R. Volpato, BPS algebras, genus zero and the heterotic monster, J. Phys. A 50 (2017) 414001 [arXiv:1701.05169] [InSPIRE].

[90] J. Polchinski, Dirichlet branes and Ramond-Ramond charges, Phys. Rev. Lett. 75 (1995) 4724 [hep-th/9510017] [INSPIRE].

[91] J. Preskill, Quantum computation, chapter 7.

[92] P. Ramond, Dual theory for free fermions, Phys. Rev. D 3 (1971) 2415 [inSPIRE].

[93] A. Taormina and K. Wendland, The overarching finite symmetry group of Kummer surfaces in the Mathieu group $M_{24}$, JHEP 08 (2013) 125 [arXiv: 1107.3834] [INSPIRE].

[94] A. Taormina and K. Wendland, Symmetry-surfing the moduli space of Kummer K3s, Proc. Symp. Pure Math. 90 (2015) 129 [arXiv:1303.2931] [InSPIRE].

[95] A. Taormina and K. Wendland, A twist in the M24 moonshine story, arXiv:1303.3221 [INSPIRE].

[96] A. Taormina and K. Wendland, The Conway Moonshine module is a reflected K3 theory, arXiv: 1704.03813 [INSPIRE].

[97] K. Wendland, Moduli spaces of unitary conformal field theories, Ph.D. thesis, University of Bonn, Bonn, Germany (2000).

[98] K. Wendland, Orbifold constructions of K3: a link between conformal field theory and geometry, Contemp. Math. 310 (2002) 333 [hep-th/0112006] [INSPIRE].

[99] R.A. Wilson, The quaternionic lattice for $2 G_{2}(4)$ and its maximal subgroups, J. Alg. 77 (1982) 449.

[100] E. Witten, Five-brane effective action in M-theory, J. Geom. Phys. 22 (1997) 103 [hep-th/9610234] [INSPIRE].

[101] E. Witten, Three-dimensional gravity revisited, arXiv:0706.3359 [INSPIRE]. 